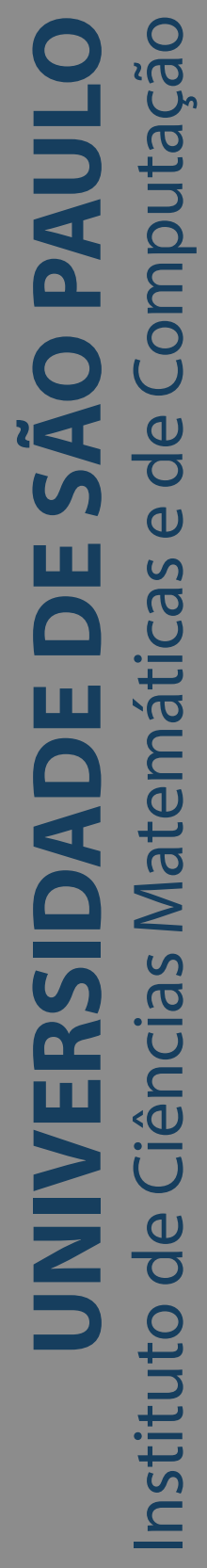

\title{
TRIVIR: A Visualization System to Support Document Retrieval with High Recall
}

\section{Amanda Gonçalves Dias}

Dissertação de Mestrado do Programa de Pós-Graduação em Ciências de Computação e Matemática Computacional (PPG-CCMC) 

Data de Depósito:

Assinatura:

\title{
Amanda Gonçalves Dias
}

\section{TRIVIR: A Visualization System to Support Document Retrieval with High Recall}

\begin{abstract}
Master dissertation submitted to the Institute of Mathematics and Computer Sciences - ICMC-USP, in partial fulfillment of the requirements for the degree of the Master Program in Computer Science and Computational Mathematics. EXAMINATION BOARD PRESENTATION COPY

Concentration Area: Computer Science and Computational Mathematics
\end{abstract}

Advisor: Profa. Dra. Maria Cristina Ferreira de Oliveira 
Ficha catalográfica elaborada pela Biblioteca Prof. Achille Bassi e Seção Técnica de Informática, ICMC/USP, com os dados inseridos pelo(a) autor(a)

Gonçalves Dias, Amanda

TRIVIR: A Visualization System to Support

Document Retrieval with High Recall / Amanda

Gonçalves Dias; orientadora Maria Cristina Ferreira

de Oliveira; coorientador Evangelos Milios. -- São Carlos, 2019.

$$
96 \mathrm{p} \text {. }
$$

Dissertação (Mestrado - Programa de Pós-Graduação em Ciências de Computação e Matemática

Computacional) -- Instituto de Ciências Matemáticas

e de Computação, Universidade de São Paulo, 2019.

1. Document retrieval. 2. Information visualization. 3. High recall. 4. Vocabulary mismatch. I. Ferreira de Oliveira, Maria Cristina, orient. II. Milios, Evangelos, coorient. III. Título. 


\section{Amanda Gonçalves Dias}

\section{TRIVIR: Um sistema de visualização para apoio à recuperação de documentos com alta cobertura.}

\footnotetext{
Dissertação apresentada ao Instituto de Ciências Matemáticas e de Computação - ICMC-USP, como parte dos requisitos para obtenção do título de Mestra em Ciências - Ciências de Computação e Matemática Computacional. EXEMPLAR DE DEFESA

Área de Concentração: Ciências de Computação e Matemática Computacional

Orientadora: Profa. Dra. Maria Cristina Ferreira de Oliveira
} 

I would like to thank my advisor Maria Cristina Ferreira de Oliveira that guided me throughout the entire project with insightful discussions, a lot of patience and friendship. Additionally, I would like to thank Evangelos Milios from the Dalhousie University that participated in this Master's project since day one with many ideas and solutions. You both made me a better researcher and a better person. I am beyond grateful for the time I spent around you.

I am also thankful for all my friends and colleagues that helped me get through this process. Your friendship and support, was crucial for me to conclude this chapter of my life. Special thanks to the USP volleyball team that was always able to pull me out of the difficult days.

I thank my family which made all of this possible. Thank you for always believing in me and for allowing me to take the opportunities life so kindly provided me so far. You are my foundation, and that is something I will never forget.

Last but not least, I thank Gustavo, who stuck with me in the good and bad days, always giving me his unconditional love and support. I love you and I will be forever grateful.

I am grateful to our collaborators Osvaldo Novais de Oliveira Jr. (IFSC, University of São Paulo), Gustavo Martini Dalpian (Federal University of the ABC) and Gustavo Martins Nunes Avellar (ICMC, University of São Paulo), who participated in the validation sessions of TRIVIR.

This work has received financial support from the Coordenação de Aperfeiçoamento de Pessoal de Nível Superior - Brasil (CAPES) - Finance Code 001. 

"We can judge our progress by the courage of our questions and the depth of our answers, our willingness to embrace what is true rather than what feels good." 



\section{ABSTRACT}

GONÇALVES DIAS, A. TRIVIR: A Visualization System to Support Document Retrieval with High Recall. 2019. 96 p. Dissertação (Mestrado em Ciências - Ciências de Computação e Matemática Computacional) - Instituto de Ciências Matemáticas e de Computação, Universidade de São Paulo, São Carlos - SP, 2019.

A high recall problem in document retrieval is described by scenarios in which one wants to ensure that, given one (or multiple) query document(s), (nearly) all relevant related documents are retrieved, with minimum human effort. The problem may be expressed as a document similarity search: a user picks an example document (or multiple ones), and an automatic system recovers similar ones from a collection. This problem is often handled with a so-called Continuous Active Learning strategy: given the initial query, which is a document described by a set of relevant terms, a learning method returns the most-likely relevant documents (e.g., the most similar) to the reviewer in batches, the reviewer labels each document as relevant/not relevant and this information is fed back into the learning algorithm, which uses it to refine its predictions. This iterative process goes on until some quality condition is satisfied, which might demand high human effort, since documents are displayed as ranked lists and need to be labeled individually, and impact negatively the convergence of the learning algorithm. Besides, the vocabulary mismatch issue, i.e., when distinct terminologies are employed to describe semantically related or equivalent concepts, can impair recall capability.

We propose TRIVIR, a novel interactive visualization tool powered by an information retrieval (IR) engine that implements an active learning protocol to support IR with high recall. The system integrates multiple graphical views in order to assist the user identifying the relevant documents in a collection. Given representative documents as queries, users can interact with the views to label documents as relevant/not relevant, and this information is used to train a machine learning (ML) algorithm which suggests other potentially relevant documents. TRIVIR offers two major advantages over existing visualization systems for IR. First, it merges the ML algorithm output into the visualization, while supporting several user interactions in order to enhance and speed up its convergence. Second, it tackles the vocabulary mismatch problem, by providing term's synonyms and a view that conveys how the terms are used within the collection. Besides, TRIVIR has been developed as a flexible front-end interface that can be associated with distinct text representations and multidimensional projection techniques. We describe two use cases conducted with collaborators who are potential users of TRIVIR. Results show that the system simplified the search for relevant documents in large collections, based on the context in which the terms occur.

Keywords: visualization, information retrieval, total recall, vocabulary mismatch, machine learning. 



\section{RESUMO}

GONÇALVES DIAS, A. TRIVIR: Um sistema de visualização para apoio à recuperação de documentos com alta cobertura.. 2019. 96 p. Dissertação (Mestrado em Ciências - Ciências de Computação e Matemática Computacional) - Instituto de Ciências Matemáticas e de Computação, Universidade de São Paulo, São Carlos - SP, 2019.

No âmbito de recuperação de documentos, há situações em que é preciso assegurar que todos os documentos relevantes para uma dada consulta serão recuperados, de preferência com um esforço humano mínimo. Uma das maneiras de formular este problema de recuperação com alta cobertura é com uma consulta por similaridade: um usuário seleciona um (ou vários) documento(s), e um sistema automático é utilizado para recuperar, de uma coleção, os documentos 'semelhantes' aos apresentados. Uma maneira usual de abordar o problema adota uma estratégia denominada Continuous Active Learning, em que dado o(s) documento(s) de consulta, descrito por seus termos relevantes, um método de aprendizado de máquina retorna e apresenta ao analista, em lotes, os documentos mais provavelmente relevantes, ou mais similares a esse(s). O analista classifica cada documento quanto à relevância, realimentando o algoritmo de aprendizado, o qual pode então refinar suas previsões. Esse processo interativo continua até que alguma condição de qualidade seja satisfeita, o que pode exigir grande esforço do usuário, já que os documentos são oferecidos no formato de listas ranqueadas e devem ser marcados individualmente, e impactar negativamente a convergência do algoritmo de aprendizado. Ademais, uma das dificuldades é a incompatibilidade de vocabulário, quando terminologias distintas são empregadas para descrever conceitos semanticamente relacionados, o que pode prejudicar a identificação dos documentos relevantes.

Neste trabalho propomos TRIVIR, uma visualização interativa alimentada por um motor de recuperação de informação (RI) que implementa o protocolo Continuous Active Learning com o fim de auxiliar RI de alta cobertura. O sistema integra várias representações gráficas para auxiliar o usuário a identificar documentos relevantes em uma coleção. Dados documentos representativos como entrada, usuários podem interagir com as visualizações e marcar documentos como relevantes/não relevantes. Esta informação é utilizada para treinar um algoritmo de aprendizado de máquina que, por sua vez, sugere documentos potencialmente relevantes. TRIVIR oferece duas principais vantagens em relação a outros sistemas de visualização para RI. Primeiro, integra a visualização a um algoritmo de aprendizado de máquina com o qual usários podem interagir para melhorar e acelerar a convergência do algoritmo. Segundo, o sistema trata o problema de incompatibilidade de vocabulário, provendo sinônimos dos termos e o contexto no qual termos são utilizados na coleção. TRIVIR foi desenvolvido como uma interface web flexível podendo ser associado com diferentes técnicas de representação de documentos e projeção multidimensional. Descrevemos dois casos de uso conduzidos com potenciais usuários do TRIVIR. Resultados 
mostraram que o sistema facilitou a pesquisa por documentos relevantes em grandes coleções, por meio da utilização da informação do contexto no qual os termos ocorrem.

Palavras-chave: visualização, recuperação de informação, cobertura total, incompatibilidade de vocabulário, aprendizado de máquina. 
Figure 1 - TF-IDF terms' distribution in a particular document from a collection of 675 documents in computer science . . . . . . . . . . . . . . . . . . 33

Figure 2 - While CBOW finds a target word $(\mathrm{w}(\mathrm{t}))$ based on its context words, Skip-gram finds the context words surround a target word $(\mathrm{w}(\mathrm{t})) \ldots \ldots . \ldots 34$

Figure 3 - The paragraph node serves as a memory that helps to find the missing information. Along with the three context words, the system's goal, in this case, is to predict the fourth word. . . . . . . . . . . . . . . . . . . . . . .

Figure 4 - Similar to the skip-gram model, the paragraph is trained to predict the words in different windows. . . . . . . . . . . . . . .

Figure 5 - Projections using different dimensionality reduction techniques of 6000 digits from the MNIST dataset . . . . . . . . . . . . . . . . . . . 40 40

Figure 6 - Projection of LSP and HiPP of 30000 data points from a news articles dataset . . .

Figure 7 - The VIBE system. Documents represented as rectangles are placed according to the keywords or POIs they contain. . . . . . . . . . . . . . . . . . . .

Figure 8 - The Visual re-ranking visualization. Red rectangles represent the keywords of interest, gray rectangles are deactivated keywords, the blue icon is the exploration cursor that will define the location to which the algorithm should use as reference to re-rank the list in (2). The circles in red in (1) represent the documents that are currently being displayed in the ranked list in (2). The circle sizes encode the overall relevance of the document to all the queries phrases. . . . . . . . . . .

Figure 9 - The TileBars system. The user can type in multiple queries and a list of documents that contain the queries' terms is presented. Each item of the list contains a visualization that shows the portions of the text that are relevant to the queries where higher the saturation, more relevant that part of the text is to the search. . . . . . .

Figure 10 - The WebSearchViz system. In (a) the user can see three subjects of interest (data visualization, software visualization and website visualization). The query is located at the center of the circle and the resulted web pages are the pink squares around it. In (b) the user sees how the results are related to a subject when s/he moves the "website visualization" subject around. It is clear that the documents in (1) are more related to "web visualization" than the documents in (2) since they barely moved.

Figure 11 - The Scatter/Gather visualization. The user is presented with an overall view of the important terms in the collection where s/he can choose some terms in order to filter the visualization to show new terms related to the selected ones. . . . . . . . . . . 
Figure 12 - The WebRat visualization. On the left is presented the results for the query "Knowledge Management". On the top right, the result after the user zoomed in into the keyword "certification" is shown and on the bottom right the user is refining the query with the word "information". . . . . . . . . . . . .

Figure 13 - The STORIES system. The main view shows a story graph of news regarding Britney Spears in 2007. The main window on the center-left represents the first story graphs resulted from the search and, as a user narrows the time window (center-right), it is possible to gain new insights about the documents since new nodes are added. If the user selects edges between the nodes, a list of pertinent documents to those nodes is presented in the center-bottom where the text can be inspected in the left. . . . . .

Figure 14 - The cit2vec system. A search for depth-based random forests techniques shows techniques and data used by documents in this domain. . . . . . . . . . . . .

Figure 15 - The VisIRR system. The user can type a query in A, see the results in both the scatterplot and in the tabular view in B, see the list of recommended documents in C, zoom-in to get more details (D) and visualize the topic cluster summary in E. . . .

Figure 16 - The Galaxy of News visualization. Documents are shown as stars in the sky. Symbols such as keywords, locations, time event(s) occurred, subjects, and actions are extracted from news articles and fed into the ARN . . . . . . . . . . . . . . .

Figure 17 - The Typograph system. The user entered the query "sun" and some phrases such as "microsoft windows" and "single released" show different contexts that the query was utilized. The user can interact further in order to visualize snippets showing the use of the query within the text. . . . . . . . . . . . . . .

Figure 18 - The BEAD visualization. Documents are represented by their IDs and the distance from the query is mapped as the color intensity of nodes. . . . . . . . . . . .

Figure 19 - The CiteSpace II system. A network of 541 co-cited authors. The thickness of each ring represent the amount of citations given a time slice and the color saturation the time of the corresponding citations. The prominent cluster (i.e. on the right) is the most recent one. . . . . . . . . . . . . .

Figure 20 - The extended Revis visualization. A system to help users with the task of Systematic Literature Review. The red circles represent articles not included in the SLR whereas blue ones are included and gray ones are cited references. . . . . . . . . . . .

Figure 21 - The Face Atlas visualization. After the search for the keyword "diabetes", the system generated two clusters representing type- 1 and type-2 diabetes. Red links represent similar complications whereas green links represent common symptoms. . . . . .

Figure 22 - The DocuCompass system. The lens puts documents in focus and groups them by color. The bar charts represent the terms' frequencies in each group. . . . . . . .

Figure 23 - The visualization with two anchors selected: the keyword "www" and the document "Finding and Visualizing Inter-Site Clan Graphs". . . . . . . . . . . . . . . . . 
Figure 24 - The Pex-Web visualization. The document are placed based on a similarity map and colors are assigned based on the frequency of the query on each document. . . . . 61

Figure 25 - (top) The basic version of the "visualization pipeline". Interaction can be performed directly on the Algorithm (blue arrow) or the data (red arrow). (bottom) Modified version of the pipeline for semantic interaction, where the user interacts within the spatial metaphor (purple arrow) . . . . . . . . . . . . . . . . .

Figure 26 - StarSPIRE spatial workspace showing clusters of open documents and numerous iconified documents selected and arranged through semantic interaction.

Figure 27 - Barchart showing the amount of Yes or No incidents for the following features: Does the solution use metadata information?, Does the solution deal with the issue of vocabulary mismatch? and Does the solution implement text classification algorithms in order to enhance the IR system? . . . . . . . . . . . . . . . . . . . .

Figure 28 - TRIVIR interface. The Scatterplot View in area (2) shows a similarity map depicting a collection of 675 papers in Computer Science. The circle colors indicate the current query document (green), and then: the relevant (blue), not relevant (red), suggested as relevant (yellow), and yet unlabeled documents (gray). The menu on top allows filtering the scatterplot in three different ways ( $2 a, 2 b$ and $2 c)$. The Terms View (1) shows the important terms from the query document, where the user can remove (1a) or add (1b) terms. Area (4) shows the Signature List view, which shows the relevant 3-grams in the corpus. It can be switched with the Focus List or the Suggestion List views by clicking on the corresponding buttons. The user can visualize important 3 -grams from the collection, select 3-grams with specific terms (4a), or select all 3-grams that include terms from the terms view $(4 b) \ldots \ldots . . . . .68$

Figure 29 - Both the Focus List view (1) and the Suggestion List view (2) share the same area in the interface, and the user switches between them (and the Signature List view) by selecting the corresponding buttons on the top (refer to Figure 28). The Focus List view shows all documents currently labeled as relevant; the Suggestion List view shows the documents suggested as relevant by the ML classifier. The user can press the "Train" button to retrain the classifier (2a). 69 
Figure 30 - Information flow in TRIVIR. The user first selects a corpus and a query document, and possibly modifies the default settings. The system initially renders the Document view and the associated Terms, Signature List and Focus List views, which are computed considering the document representation, the creation of a model containing the word representation of the collection using the skipgram model, the ranked 3-gram list, and the distance function. The ML algorithm is trained; the documents it predicts as relevant define the Suggestion List view. Finally the 2D Scatterplot view is created and rendered, with the circles colored according to the documents' labels. Interacting with the multiple views the user has the necessary support to investigate the collection and decide about document relevance. The CAL protocol allows her to retrain the ML classifier and finish the process at any time, when the final Focus List view gives all the documents identified as relevant. . . . . . . . . . . . . . . . . . . .

Figure 31 - List of synonyms for the term "case". The list merges dictionary synonyms and terms that are used in the same context as the selected one in the collection. 72

Figure 32 - Using the Signature List view to filter the scatterplot to display the documents that include the 3-gram "case base reason". One observes four categories of documents in the Scatterplot view (all of them include this 3-gram): the query document (green), relevant documents (blue), suggested documents (yellow) and not yet labeled documents (gray). The user can set all not labeled documents as not relevant (a) or relevant (b) at once. . . . . . . .

Figure 33 - The menu on the Scatterplot view has different options depending on the label of the document. For not labeled (gray) and suggested documents (yellow) the user has the option of setting a document as relevant or not relevant (a). For relevant documents (blue) the user can set it as not relevant or query (b) and for not relevant documents (red) the user can add them to the Focus list (set as relevant) or set other documents similar to it as not relevant $(\mathrm{c}) \ldots \ldots \ldots 74$

Figure 34 - Demonstration of how to expand the set of relevant (by creating additional query documents) and not relevant documents. The example illustrates the before and after setting a document as query ( $a$ and $b$ ) and the before and after expanding the set of not relevant documents $(\mathrm{c}$ and $\mathrm{d}) \quad \ldots \ldots \ldots$. . . . . . . . . . . 75

Figure 35 - Suggestion View menu. The user has the options to set a document as not relevant or relevant. . . . . . . . . . . . . . . . . .

Figure 36 - Focus List menu. The user has the option to remove the document from the list. 76 
Figure 37 - Scatterplot projections of a corpus with 1,363 papers, both created with the t-SNE projection technique and different document vector representations, namely word embeddings in (a); the VSM and a TF-IDF weighting scheme in (b). Blue circles correspond to documents currently labeled as relevant, yellow to documents suggested as relevant, not relevant documents are in red and the query documents in green. . . 80

Figure 38 - After searching for the word "cell" in the signature view, the user realized it is used in different contexts in the collection. Thus, he scanned the list and set all documents containing interesting 3-grams as relevant. . . . . . . . 80

Figure 39 - Distribution of labeled documents at the end of the SMS session. . . . . . . 83 

Table 1 - Some of the solutions presented in the related work section regarding the A Category (1: Understanding the search; 2: Understanding the corpus; and 3: Understanding the search and the corpus), B - if the proposed visualization requires metadata, $\mathrm{C}$ - if the solution counted on text classification in order to enhance the IR system and D - If the solution handled the vocabulary-mismatch issue. . . . . . . . . . . . . . . . . . . . 64 



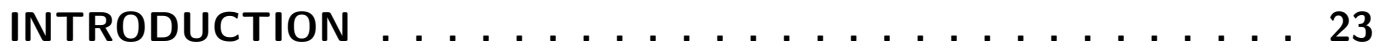

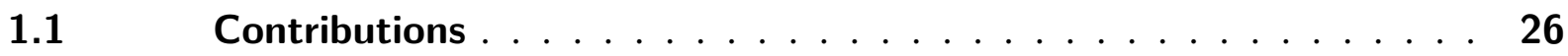

$1.2 \quad$ Structure of the document $\ldots \ldots \ldots \ldots$

2 DOCUMENT RETRIEVAL . . . . . . . . . . . . . . 27

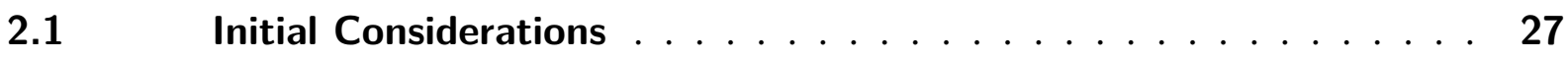

$2.2 \quad$ Document Retrieval . . . . . . . . . . . . . . . . . . . 27

2.2.1 Retrieval Models . . . . . . . . . . . . . . . . . . . 28

2.2.2 Active learning in information retrieval . . . . . . . . . . . . . 28

2.2.3 Challenges in Document Retrieval . . . . . . . . . . . . . . . . . 29

$2.3 \quad$ Document Representation . . . . . . . . . . . . . . . . . 30

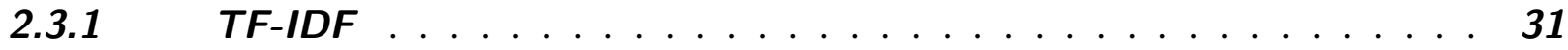

2.3.2 Word Embeddings . . . . . . . . . . . . . . . . 33

$2.4 \quad$ Text Classification . . . . . . . . . . . . . . 36

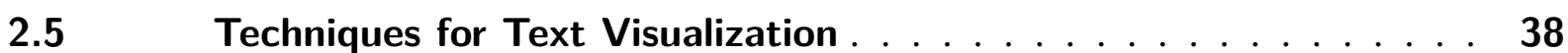

3 INFORMATION VISUALIZATION FOR INTERACTIVE TEXT RETRIEVAL . . . . . . . . . . . . . . . . . 43

$3.1 \quad$ Understanding the search $\ldots \ldots \ldots \ldots$

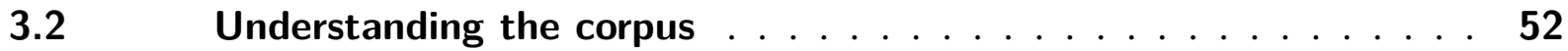

$3.3 \quad$ Understanding the search and the corpus . . . . . . . . 58

3.4 Discussion and final considerations . . . . . . . . . . . 63

4 TRIVIR: A VISUALIZATION SYSTEM TO SUPPORT DOCUMENT RETRIEVAL WITH HIGH RECALL . . . . . . . . . . . . . . . 67

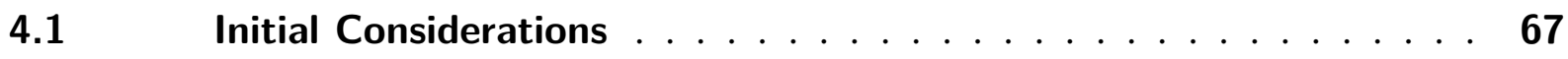

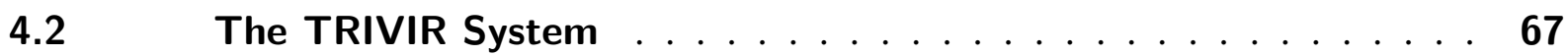

4.2.1 Tasks supported . . . . . . . . . . . . . . . . . . . 69

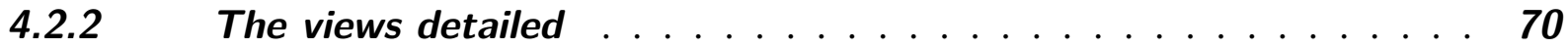

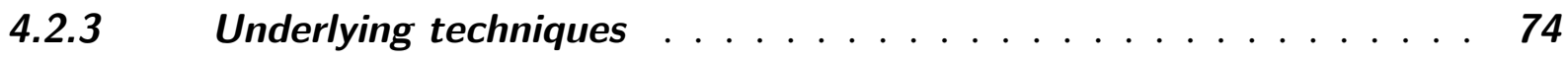

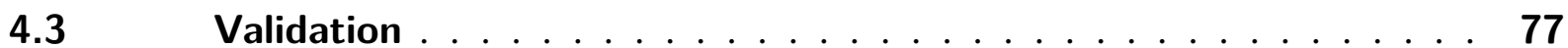

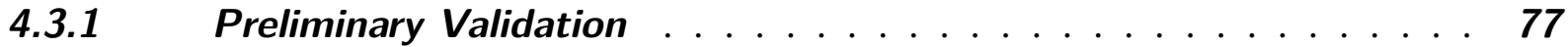

4.3.1.1 Data and system setup . . . . . . . . . . . . . . . . . . 77

4.3.1.2 Results ...................... 78 
4.3.2 A literature review problem . . . . . . . . . . . . . . 79

4.3.2.1 Data and system setup . . . . . . . . . . . . . . . . 79

4.3.2.2 Results . . . . . . . . . . . . . . . . . . . 79

4.3.3 A Systematic Mapping Study (SMS) scenario . . . . . . . . . . 81

4.3.3.1 Data and system setup . . . . . . . . . . . . . . . . . . 81

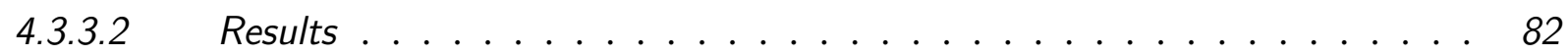

$4.4 \quad$ Final considerations $\ldots \ldots \ldots \ldots \ldots \ldots$

$5 \quad$ CONCLUSIONS $\ldots \ldots \ldots \ldots \ldots$

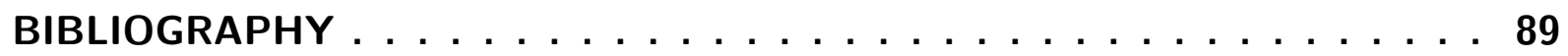


CHAPTER

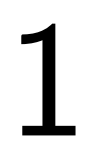

INTRODUCTION

The wide availability of resources for the bulk digitalization of documents and other media produces vast collections of digital material of interest to professionals and researchers in the human and social sciences. This scenario increases the demand for the development of computing technologies to support investigations on such collections in general, and collections of textual documents in particular. There are potential applications in many areas, e.g., medical, social and legal, encompassing a variety of problems, tasks and goals that require analyzing large document corpora.

Research on Visual Analytics is central to addressing the current challenges associated with data analysis and data-centered discovery. Visual Analytics (VA) (OLIVEIRA; LEVKOWITZ, 2003; WONG; THOMAS, 2004; KEIM et al., 2008) refers to the integration of Machine Learning and Data Visualization techniques to assist humans in tasks that require the interpretation of complex data. VA advocates a strong coupling of techniques from both areas to advance data analysis capability, by giving human and computer complementary roles in handling the many issues introduced by the volume and complexity of current data sets. VA is particularly relevant in handling complex multidimensional data, including collections of textual documents.

Given a set of documents, users may be interested in finding within this corpus documents related with certain topics (BLAIR; MARON, 1985). The documents of interest are called "relevant", i.e., they satisfy the user's information need, whereas the others are named "not relevant". If the user is searching for a specific piece of information, the information need can be expressed clearly and unambiguously as a query. In this scenario, the classic ranked list of hits returned by search engines usually suffices. However, a different situation arises when a user can not concisely and objectively express a query, but it is still crucial that s/he finds the relevant documents to her information need. A typical example is when users are surveying the literature on a certain topic. An initial keyword-based search may return many documents, but not all of them will be actually relevant to the survey. User effort is necessary to identify 
the relevant ones. Recall and Precision are often employed as measures of the success of an Information Retrieval (IR) task. Recall is the proportion of relevant documents in the corpus that were actually retrieved, whereas precision is the proportion of retrieved documents that are actually relevant.

Our contribution focuses on information retrieval tasks that demand high recall. Such tasks occur frequently in diverse situations and application domains. Possible scenarios include, for example, lawyers searching for previous cases that may be in some way related to a current one; researchers engaged in a literature survey who do not want to miss relevant studies to their topic; medical doctors searching for patient records that may include medical conditions relevant to a case under study; or people performing patent search where missing a single relevant patent can result in invalidation of an otherwise sound patent. Pursuing high recall, however, often results in loss of precision, meaning the user will end up retrieving many not relevant documents. This is a crucial issue, as we shall observe in the results section (Section 4.3), where we discuss our solution applied on a real-word scenario.

An illustrative example of the potential of text analytics in practical applications in humanities comes from the legal domain. In countries that follow the Common Law legal system, e.g., Canada and the United States, once a lawsuit gets underway, the parties or their lawyers start an investigative process named "discovery". This is a formal and strongly regulated process, in which the goal is to gather information related to the lawsuit, finding out facts and documents previously unknown (to one or both parties) that may be relevant to the dispute. Currently, Electronic Discovery (or E-Discovery, for short) refers to the discovery process conducted on Electronically Stored Information (ESI), which may include emails, documents, presentations, databases, voice mail, audio and video files, social media, and web sites. Typically, parties need to review documents in order to identify responsiveness (relevance to a specific information need), or privileges, i.e., documents that should be withheld due to privacy regulations.

Analytic software to support E-discovery often rely on Technology Assisted Review, referred to as TAR Cormack and Grossman (2013). Using TAR for E-discovery can reduce the number of documents required for review by attorneys, with an impact on labor effort and costs. It has been shown (GROSSMAN; CORMACK, 2011) that a supervised machine learning TAR method is more effective than human review, as measured by recall and precision. Nonetheless, a major concern in this scenario is minimizing the risk of overlooking important documents. This example illustrates a typical scenario of total recall in information retrieval, i.e., given a particular information need (expressed as a query, or as one or multiple representative documents), a system should be able to ensure that nearly all relevant documents to the query will be retrieved.

The legal domain is just one example. A similar scenario has been investigated by Makki et al. (2017) in another domain: their goal is to retrieve Twitter posts related to a predefined set of topics, and label them accordingly. The topics, in this case, describe relevant political debates held at the Canadian Parliament. The retrieval system is potentially useful, e.g., to 
investigative journalists or social researchers, and similarly to the previous case a major concern is that relevant posts are not missed - otherwise analysts might be misled or biased in their studies. Again, there is a growing demand for tools to support analytics of social media posts. Nonetheless, ensuring that relevant posts are not missed is not a trivial task, due to the sheer volume of posts, the specificity of the debates, and the noisy and inconsistent vocabulary adopted in Twitter.

Both examples illustrate situations that lead to the formulation of a high recall problem in the context of textual document retrieval, and several other scenarios could be listed: document retrieval with high recall is a problem that has received considerable attention from the collaborators at the MALNIS research group from University of Dalhousie, Canada. The problem can be expressed in different manners, but our interest lies in scenarios in which it is expressed as a document similarity search: user picks an example document (or multiple ones), and wants an automatic system to recover similar ones in the collection, based on the informative keywords, or terms, identified in the query document.

A strategy often employed in scenarios similar to the ones just described is referred to as Continuous Active Learning (CORMACK; GROSSMAN, 2014): once presented to an initial query, a learning method returns the most-likely relevant documents (e.g., the most similar) to the reviewer in batches, the reviewer labels each document (as relevant/not relevant) and the labels are fed back to the learning algorithm which uses this information to refine its predictions. This iterative process goes on until some condition is satisfied - e.g., no more improvements observed in a target quality measure.

The interactive process is a great tool in scenarios where high recall is required because users are often more willing to provide feedback and interact with the information retrieval system, as opposed to users that are interested in very high precision and usually count on web search engines.

In a general setting, one of the difficulties faced stems from the vocabulary mismatch issue, i.e., the use of different terminologies to describe semantically related or equivalent concepts. The challenge is to identify the semantically related terms when providing relevance feedback to a learning algorithm, so that the corresponding related documents are also identified.

This scenario provides the motivation for this research proposal, in which the aim is to investigate the following research problem: "Can an user interacting with appropriate visual representations of a document collection improve total recall in a (general) document similarity search?" 


\subsection{Contributions}

Within the presented context, our studies resulted in TRIVIR, an interactive visualization tool for information retrieval with high recall, i.e., we want to ensure that very few relevant documents will be missed by the retrieval task. As described in Chapter 4, it integrates a front-end visualization interface to a back-end information retrieval engine associated with a machine learning (ML) algorithm, which learns how to identify relevant documents based on user input. The ML algorithm suggests potentially relevant documents that are shown as a ranked list and also mapped in a Scatterplot view where one can see how suggested documents are related with other documents in the collection. The user can interact with multiple graphical views to explore the collection in order to identify and label documents that meet her information needs. TRIVIR offers two major advantages over existing visualization systems for information retrieval. First, it merges the ML algorithm into the visualization while allowing several user interactions in order to enhance and speed up its convergence. Second, it tackles the problem of vocabulary mismatch by providing synonyms of terms and a view that shows the context where a term occur. Besides, TRIVIR is a flexible interface over different text representations and multidimensional projection techniques.

\subsection{Structure of the document}

This document is organized as follows. Chapter 2 describes a short background on document retrieval. A brief survey on related work on text visualization is presented in Chapter 3. The TRIVIR system as well as our validation exercises are described in Chapter 4. Finally we present our conclusions in Chapter 5. 
CHAPTER

2

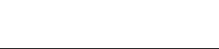

\section{DOCUMENT RETRIEVAL}

\subsection{Initial Considerations}

In this work, we deal with concepts commonly used in the information retrieval field. The following sections provide an overview of the techniques and nomenclature used throughout this dissertation. More specifically, this chapter covers studies in the document retrieval area, including usage scenarios and challenges. Additionally, it covers aspects such as document representation, dimensional reduction and text classification techniques.

\subsection{Document Retrieval}

The historical need of human beings to gather information became even more pressing after the introduction of computers. According to Blair and Maron (1985), given a set of documents by different authors and collected at different moments, users might want to find documents related with certain topics of interest within this set. The documents of interest are called "relevant", whereas the others are named "not relevant". Document retrieval was for many years a manual process. However, the availability of huge amounts of digital document sets made it unfeasible to search for relevant documents without computer assistance. Computerized full-text document retrieval emerged in this context, introducing new approaches, challenges, and many novel applications going from simple web search to governmental and institutional systems. New methods and techniques have been created in order to enhance the search experience moving from simple lists of ranked documents to more complex visualizations and algorithms.

A document can be considered relevant to a query based on two major aspects, namely (1) topical and user constraints and (2) whether binary or multivalued relevance is considered. The topical relevance could be measured based on the match between a document and the query's terms. On the other hand, relevance also takes into consideration the user judgment, which could include aspects such as the document's date or language, for example. The second 
aspect concerns binary relevance, meaning that a document is either relevant or not relevant. However, if multivalued relevance is considered, a document may have more than two levels of relevance. Retrieval models arose to provide a framework for defining new tasks and explaining assumptions. Classic retrieval models are the Boolean model and the Vector Space model (CROFT; METZLER; STROHMAN, 2009).

\subsubsection{Retrieval Models}

The Boolean Retrieval model uses Boolean expressions to formulate a query. When searching for documents, the Boolean model will consider a document relevant only if it matches the query entirely. Documents are not ranked by relevance, since all relevant documents are treated as equals. This model is easy to interpret, which brings transparency to the retrieved set, and it is more efficient than ranked retrieval models. However, it requires a certain level of user expertise, since the lack of a ranking algorithm makes simple queries less effective (CROFT; METZLER; STROHMAN, 2009).

The Vector Space model applies term weighting, ranking and possibly relevance feedback in order to enhance search effectiveness. It represents queries and documents as $t$-dimensional vectors defined in a multidimensional space, where $t$ is the number of index terms. A common method to determine whether a document is relevant to a query and its degree of relevance, is to compute the cosine correlation on the query and documents normalized vectors. This model is not as transparent as the Boolean model, as it may be difficult to understand why documents have been considered relevant and how the rankings have been applied (CROFT; METZLER; STROHMAN, 2009).

The facet query can be seen as a combination of both previous models. This technique allows users to perform multiple Boolean queries simultaneously and documents are ranked based on the number of matched queries (SINGHAL, 2001). By providing multiple searches at once, users can analyze different perspectives of a search.

\subsubsection{Active learning in information retrieval}

Active Learning (AL) appears in contexts where a ML algorithm is allowed to choose interesting documents to be labeled, e.g., to create a training set for classification when labeled data is scarce. AL aims at achieving high accuracy while minimizing the cost of obtaining labeled data (SETTLES, 2009). It is particularly relevant in certain IR scenarios in which there are many unlabeled items and a user interested in a small portion of the collection. The user, in this context called an 'oracle', is presented unlabeled items (also known as training points, or queries) to label. Beyond recommending items likely of interest, as in Recommender Systems, the rationale is to let the user understand her preferences while feeding an algorithm that considers this information in providing subsequent recommendations, thus personalizing the recommendation 
process (RUBENS; KAPLAN; SUGIYAMA, 2011).

The legal field makes intensive usage of document retrieval, having introduced the term "electronic discovery" or "e-discovery", defined as the process of identifying, preserving, collecting, processing, searching, reviewing, and producing Electronically Stored Information that may be relevant to people from different scenarios and backgrounds. (CORMACK; GROSSMAN, 2013). In this context, technology-assisted review ("TAR") has been introduced to support ediscovery. According to Cormack and Grossman (2014), its purpose is to find as much relevant documents in a collection as possible, with reasonable effort. The most frequent approach for TAR is to use a training set to select certain terms or phrases that characterize a document as relevant/not relevant. Then, the classifier algorithm predicts whether documents outside the training set should or not be useful (CORMACK; GROSSMAN, 2016b). Algorithms considered effective include Support Vector Machines, Logistic regression, Nearest Neighbor and Naive Bayes. These can be associated with multiple TAR protocols (GROSSMAN; CORMACK, 2016), such as the Continuous Active Learning (CAL) protocol adopted in TRIVIR. The CAL protocol comprises four steps, namely:

1. Create an initial set of possible relevant documents.

2. Train a ML algorithm with the labeled documents. This step should return a ranked list with documents that might be relevant to the user. It is common for the ML algorithm to use binary relevance.

3. The user can provide relevance feedback to the system by labeling documents as "relevant" or "not relevant".

4. Repeat steps (2) and (3) and, if necessary, also step (1), until no more (or very few) relevant documents are suggested by the classifier (GROSSMAN; CORMACK, 2016).

Unlike other protocols such as Simple Active Learning (SAL) and Simple Passive Learning (SPL), CAL does not face the problem of determining when to stop training and how many documents should be included in the review set. However, the question of when to stop the search still holds (CORMACK; GROSSMAN, 2016a).

\subsubsection{Challenges in Document Retrieval}

When evaluating the outcome of a search, the most usual measures are recall and precision. Recall is defined as "the fraction of relevant documents that are identified as relevant by a search or review effort"; Precision is "the fraction of documents identified as relevant by a search or review effort, that are in fact relevant." (CORMACK; GROSSMAN, 2013). It is expected that the best search results would maximize recall and precision, however, what usually happens is that high recall results in low precision and vice-versa. According to Manning, 
Raghavan and Schütze (2008), a Boolean search tends to yield low precision and high recall where OR operators are used, and low recall and high precision where AND operators are used. This situation makes finding a satisfactory middle ground very difficult or impossible.

An influential study by Blair and Maron (1985) has shown that the system STAIRS, a state-of-art software in full text retrieval at the time, was capable of retrieving only 20 percent of the relevant documents, while its users (lawyers) believed it had achieved over 75 percent. A reason for the low recall is that words or phrases could be written in different ways but still own the same meaning. As an example, the word "accident" might be reported in a legal or news document using the keywords "event", "incident", or "situation". Besides, misspelling also proved to be an obstacle when retrieving similar documents. That study proved that many early techniques for document retrieval were unsuitable for large document sets. The concern for providing scalable techniques motivated the creation of the Text Retrieval Conference (TREC) in 1992, that expanded the studies on information retrieval (IR) for large text collections (SINGHAL, 2001).

Several challenging issues remain in document retrieval. One of them is determining relevance, since it is not straightforward to assess document relevance based solely on the user's query. Several document retrieval models have been created in order to address this issue which are a formal representation of the process of matching a query and a document (CROFT; METZLER; STROHMAN, 2009). Retrieval evaluation is an additional challenge. Whilst recall and precision are popular measures, they are difficult to measure on a web search environment and are normally evaluated on small text collections. Moreover, as stated by Baeza-Yates and Ribeiro-Neto (1999) even though recall and precision are widely employed to compare the result set of non-interactive systems, they are less appropriate for assessing interactive systems. Novel metrics are necessary, such as the time required to learn the model, the time required to achieve goals on benchmark tasks, error rates, and retention of the interface usage over time.

Finally, another core issue is how to best determine a user's information needs, which is not always well defined. For this, several techniques, including query suggestion, query expansion and relevance feedback have been introduced in order to help users express their needs properly and improve the document retrieval process (CROFT; METZLER; STROHMAN, 2009).

\subsection{Document Representation}

Since computers cannot make sense of actual text, some processing is needed. A common approach is to transform a text document into a vector representation. This vector might simply be the count of the terms within a document (commonly referred to as bag-of-words or the Vector Space Model), or some additional processing can be applied in the vector in order to give more importance to representative terms. Representing documents in a vector space, is known 
as the vector space model, and it is really useful in other information processing tasks, such as document classification, document clustering and document ranking (MANNING; RAGHAVAN; SCHüTZE, 2008). Documents are represented as points in the vector space and the distances among points (i.e. documents) depict their semantic similarity (TURNEY; PANTEL, 2010). Additionally, some pre-processing is usually necessary to avoid including in the vector common words such as "of" and "the". This process is called stop-word removal and consists of removing certain words devoid of meaning from the document before representing it as a vector. A stopword list might contain general common words or domain specific terms such as "conference" or "abstract" if one is handling scientific documents, for instance. Also common is to remove special characters and numbers when appropriate.

Additionally, some normalization such as transforming all the words into lower case characters and uniformly handling different forms of a word can be applied in the pre processing step. For example, the words "analyse", "analyses" and "analysed" should be interpreted as having the same meaning and appear as a single term in the vector representation. In order to achieve this goal, stemming or lemmatization can be applied, where the former is a simpler solution where common suffixes are removed. In this case, all the three mentioned words would become the word "analys". However, dealing with the words "see", "sees" and "saw", the simplest stemming algorithm would reduce all forms to "s". More advanced stemming algorithms would have a minimum number of letters to keep, which would properly change "sees" to "see", however, they would not modify the word "saw". Lemmatization is a more elaborate process where the words are transformed into their root or dictionary form. Thus, the first group of words would be transformed into the single word "analyse" and the second group would be represented by the word "see".

Other techniques can be used in order to retrieve the most important aspects of a document, such as tokenization, which consists of retrieving the most important terms (called tokens) of a document, where each token might consist of multiple words. For example, the words Air Canada usually appear together, because they represent the name of a company, thus they represent a single token. Additionally, metadata from specific domains that do not bring representative value can be removed, for example author's names, university names, and dates in a corpus of scientific papers.

The following sections describe some techniques used to represent documents. More specifically, how the vector representation can be enhanced in order to better represent the singularities of each text in a document corpora.

\subsubsection{TF-IDF}

A problem with the bag-of-words approach is that the raw frequency of terms does not represent the real importance of a term to a document when assessing its relevancy to a query. For example, the word "computer" would have a high frequency in a computer science collection and 
thus, does not help an algorithm to assess the relevance of a specific document to a query. High frequent terms can be removed from the collection, however, even though these terms might be considered noise, they may be also required for achieving reasonably high recall (JONES, 1988). Similarly, we also want to assign proper importance to the low frequent terms that are helpful in discriminating a document's relevancy, which implies that some sort of weighting is necessary. This is the motivation for the TF-IDF weighting scheme.

TF-IDF is split into two components: the term's frequency (TF) and the inverse document frequency (IDF). The first component is straightforward and is the raw frequency of the term within the document. For the second part, first we compute the document frequency $\left(D F_{t}\right)$ which is the number of documents from the collection that contain the term $t$. Then, the IDF of a term $t$ is as follows:

$$
I D F_{t}=\log \frac{N}{D F_{t}}
$$

Where $\mathrm{N}$ is the total number of documents in the collection. Equation 2.1 suggests that the IDF of a rare term is high whereas the IDF of a frequent term is low (MANNING; RAGHAVAN; SCHüTZE, 2008). Finally, the TF-IDF weighting scheme can be applied to each term $t$ in each document $d$, as in Equation 2.2:

$$
T F I D F_{t, d}=T F_{t, d} \times I D F_{t}
$$

According to (MANNING; RAGHAVAN; SCHüTZE, 2008), the TF-IDF weight assigns high weights to terms that are popular in a small number of documents, whereas low weights are assigned to terms that have very low frequencies within documents, and the lowest weights are assigned to a term that appear in most documents from the collection. Figure 1 illustrates the TF-IDF values for a particular document from a collection of 675 papers in four computer science subjects namely: Case-Based Reasoning (CBR), Inductive Logic Programming (ILP), Information Retrieval (IR) e Sonification (SON). In this example, the document describes a software that presents ethical dilemmas to a user about whether to tell the truth. Thus, the terms with the highest TF-IDF values are "moral" and "ethics" whether the terms with the lowest TF-IDF are "in" and "of". Notice that sometimes the terms with the highest TF-IDF are not really meaningful. For example, an author's name might have a high weight but it is not necessarily a representative term for characterizing the document.

Even though there are other approaches to assign weights to terms in a bag-of-words representation, TF-IDF is the most popular (TURNEY; PANTEL, 2010). Some extensions were proposed, such as including synonyms information into the IDF calculation in order to retrieve more unique keywords from documents (KUMARI; JAIN; BHATIA, 2016). 
Figure 1 - TF-IDF terms' distribution in a particular document from a collection of 675 documents in computer science

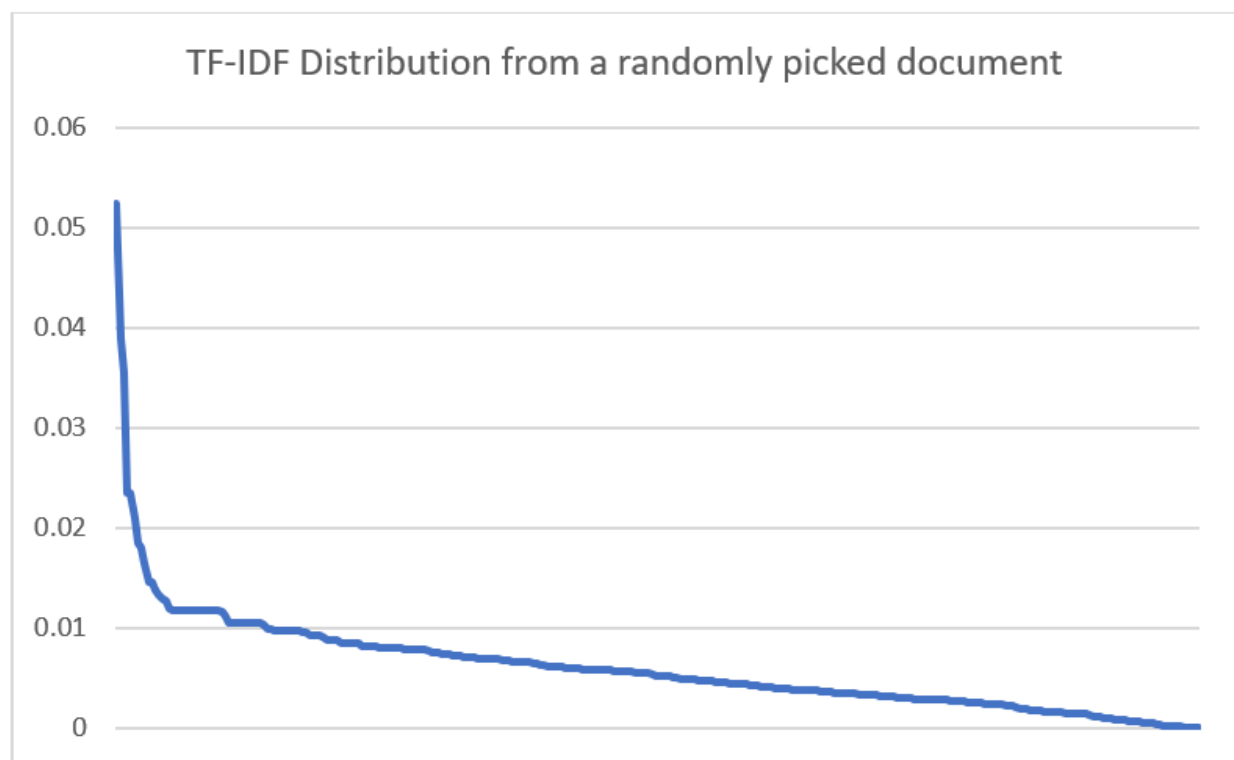

Source: Developed by author

\subsubsection{Word Embeddings}

Even though the bag-of-words representation and its variations are highly popular, they are not suitable in some information retrieval scenarios, since it does not consider the inherent relationships among terms in the text. Besides, the bag-of-words representation ignores word order, which means that completely different phrases formed by the same words would be equally represented, with a possible negative effect.

In order to mitigate the above problems, methods that use neural networks to create vector representation of words have been introduced. The study by Mikolov et al. (2013) presented the Skip-gram model, which became the base model for many following techniques including word2vec (MIKOLOV et al., 2013), a now highly popular technique to create word representations.

Advances in data availability and computing power made possible to train machine learning algorithms on much larger data sets which typically outperform simple models such as the bag-of-words. Mikolov et al. (2013) presented two new architectures to build vector representations of words illustrated in Figure 2. The first one is the Continuous Bag-of-Words Model (CBOW), which given the words surrounding a target, predicts the target word. The second model is the continuous Skip-gram model, which is also a neural network where given an input word, the model predicts the most likely surrounding words. The models are considered simple because, unlike common neural network models, the hidden layer has a linear function. This feature might not represent the data too precisely, but it allows for efficient training on much larger data sets. 
Figure 2 - While CBOW finds a target word $(\mathrm{w}(\mathrm{t}))$ based on its context words, Skip-gram finds the context words surround a target word $(\mathrm{w}(\mathrm{t}))$.

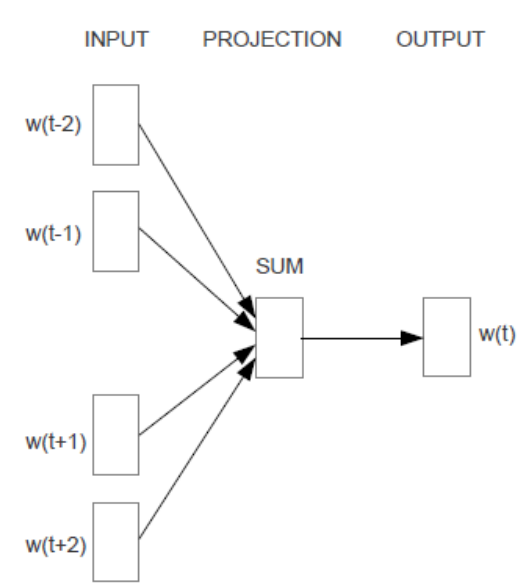

своW

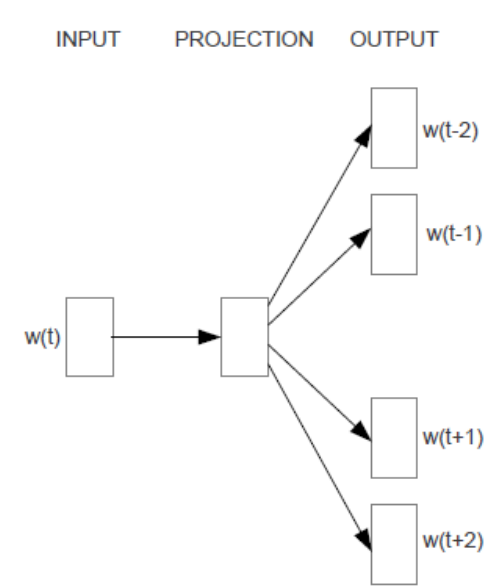

Skip-gram

Source: Mikolov et al. (2013)

This kind of representation allows executing simple algebraic operations in the vectors and thus, finding inherent relationship among words. For example, it was found that w("Paris") $w(" F r a n c e ")+w(" I t a l y ")$ is very close to the vector w("Rome") where w(word) means the vector representation of a specific word. Additionally, beyond obtaining the model, one also has a word representation that can be employed in different tasks (MIKOLOV; YIH; ZWEIG, 2013).

In order to enhance the skip-gram model, Mikolov et al. (2013) introduced several extensions that resulted in word $2 \mathrm{vec}$, an open source project ${ }^{1}$ that incorporates both the CBOW and the Skip-gram models. The original softmax function in the hidden layer was replaced by two new functions: the hierarchical softmax and the negative sampling. The main advantage of the hierarchical softmax is that instead of evaluating all words in the vocabulary in the output nodes, it evaluates around $\log _{2}(W)$ nodes, where $\mathrm{W}$ is the total number of words in the collection. On the other hand, the underlying rationale of negative sampling is that given an input word and given the positive output words returned by the model, the algorithm chooses some negative samples according to a certain probability and updates the weights of the negative samples and the positive words, instead of the entire vocabulary. Negative sampling has proved to be accurate in learning representations, specially for frequent words. Moreover, in order to counter the imbalance between rare and frequent words, a sub sampling approach was used where each word in the training set is discarded with a certain probability. Using the skipgram model as base model, Bojanowski et al. (2017) proposed a model that represents each word as a bag of character $n$-grams. The underlying rationale is that word representations can be improved if words are represented by the sum of their character n-gram vector representations, specially for morphologically rich languages. For example, taking $n=3$ and the word "where", the algorithm

1 code.google.com/p/word2vec 
would set as input: <wh, whe, her, ere, re $>$ and the full word "where". The model performed better on languages such as Arabic, German and Russian than English, French or Spanish. However, it also proved effective when dealing with the English Rare Words dataset. This model is available in the "fastText" website ${ }^{2}$, which also provides a model for text classification.

After the development of word embeddings, some studies were conducted in order to create similar representations of sentences, paragraphs and even full documents. One of the most popular solutions is the doc2vec method, introduced by Le and Mikolov (2014) under the name of Paragraph Vectors. The method embeds two models that can represent texts varying from sentences to full documents. The first one is called the Distributed Memory Model of Paragraph Vectors (PV-DM) and consists in concatenating a paragraph with word vectors and predicting the following word in the given context. In this method, the paragraphs are mapped to a unique vector and are represented as a column in a matrix $\mathrm{D}$, whereas words are mapped in a column in matrix W (see Figure 3). Then, paragraph and word vectors are averaged or concatenated in order to predict the next word in a context. It has been found that concatenating the vectors results in better predictions. The paragraph can be seen as a word that acts as a memory to remember which words are missing from the current context or topic.

Figure 3 - The paragraph node serves as a memory that helps to find the missing information. Along with the three context words, the system's goal, in this case, is to predict the fourth word.

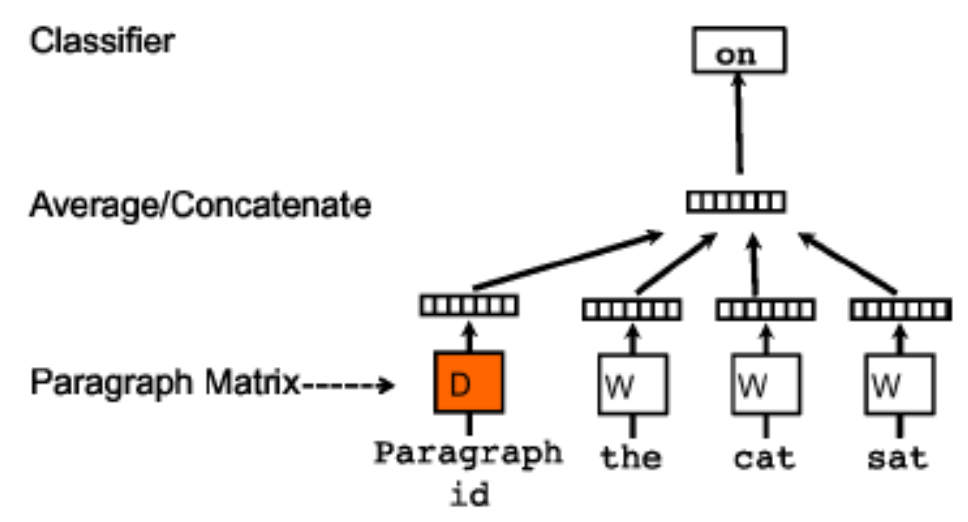

Source: Le and Mikolov (2014)

The second model is called Distributed Bag of Words version of Paragraph Vector (PVDBOW) and its goal is to predict words from the paragraph (Figure 4). The idea is to randomly select a word from a text window in the paragraph and use the algorithm to predict its surrounding words given the paragraph context (the selected window). This model requires less memory than the previous one and is similar to the skip-gram model.

The paper reports experiments conducted using a combination of the two models, however, it has been found that using only the PV-DM model generates satisfying results for most 
Figure 4 -Similar to the skip-gram model, the paragraph is trained to predict the words in different windows.

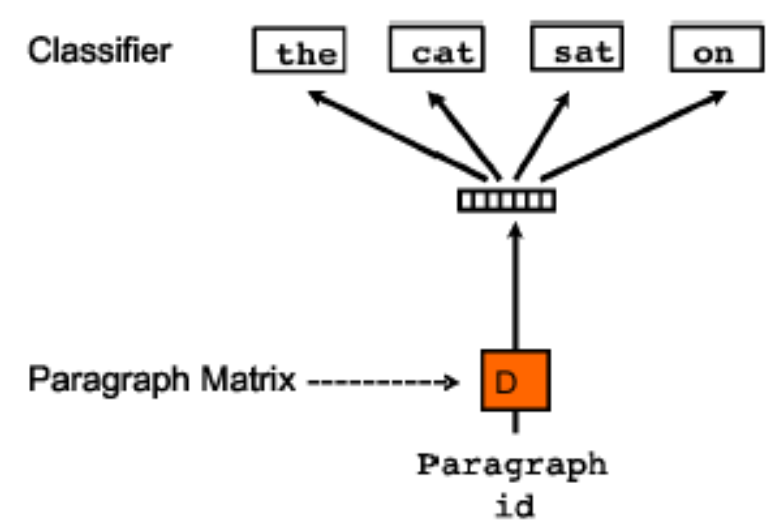

Source: Le and Mikolov (2014)

tasks. Experiments in text classification using the proposed method were conducted in two data sets, namely the Stanford Sentiment Treebank dataset that contains 11,855 sentences from movie reviews, and the IMDB dataset, which consists of 100,000 movie reviews including several sentences. The method has also been tested on an information retrieval task. The results confirmed that PV-DM achieved lower error rates than existing ones.

The goal of the information retrieval experiment consisted in finding two out of three snippets retrieved from a web engine that were related to a given query. The rationale is that given the distance among the paragraphs, the two that were actually related to a query would be closer, whereas the unrelated one would be far apart. Results have shown that the new method achieved the lowest error rate compared to others, including the bag-of-words model using the TF-IDF weighting scheme (3.82\% and $8.10 \%$, respectively).

Even though word embeddings learn the context of a given word, problems remain due to polysemy, that implies that one word may have different meanings depending on the context, since the model learns a single representation for each word. Further studies (ZAMANI; CROFT, 2017) and (KIELA; HILL; CLARK, 2015) try to remedy this issue by adding semantic information to the model according to the user needs.

\subsection{Text Classification}

In Section 2.2 we briefly described the CAL protocol and its importance to information retrieval tasks, specially when the goal is to achieve high recall. The rationale is to present to the user the top K most similar documents to a query document so s/he can label the results as "relevant" or "not relevant". Then, these labeled documents are used to update a classifier model that will come up with new documents for the user to review. The process can stop according to several rules, such as when the user considers appropriate, when no new documents are 
considered relevant by the algorithm, or when all the documents have been labeled. This later option will achieve the highest recall, at the cost of greater human effort.

Text classification algorithms use a vector representation of text as input, and return a class or category of the text. The output can be binary, such as "relevant" or "not relevant", 1 or 0 , or handle multiple categories such as "not relevant", "neutral", "relevant" or lie between a range, e.g., 1-5 stars in a movie review. In order for the algorithm to know the class of a text, it needs to be trained with labeled data. This is the motivation for employing supervised machine learning algorithms and neural networks for automatic text classifications tasks (KADHIM, 2019).

Even though neural and deep networks usually provide better results than standard machine learning algorithms (KAMATH; BUKHARI; DENGEL, 2018), they are computationally expensive and usually require a large training set to produce good results. For these reasons standard text classification algorithms remain very popular since they require less resources and usually yield satisfying results. Popular algorithms include Support Vector Machine (SVM), Logistic Regression and Naive Bayes classifiers.

A problem with classification algorithms is that they do not share parameters among features and classes, which limits their generalization power when dealing with a large output space and few training samples (JOULIN et al., 2017). In order to tackle this problem, Joulin et al. (2017) presented a text classifier, embedded in the "fastText" library, which can train on a billion words in ten minutes and achieves performance comparable with state-of-the-art solutions. The underlying rationale of the "fastText" classifier is to find vector representations for text and labels so that labels associated with certain texts appear close together in the vector space. This is done using a similar architecture to CBOW. The hierarchical softmax function is used to compute the probability of a correct label, given an input text. The score returned by the softmax function is normalized with the scores returned for the same function using other labels. Additionally, instead of using the bag of words representation that ignores word order, the algorithm uses bag of n-grams to capture partial information regarding word order.

Experiments were conducted in two different tasks. The first one considered the problem of sentiment analysis to compare fastText with existing text classifiers, and the second one used a tag prediction dataset in order to evaluate its capacity to scale to large amounts of data. The results have shown that fastText achieved results on par with recent deep learning inspired methods, with the advantage of being much faster.

The goal of this study is not to find the best text classifier algorithm, but rather check its usefulness when applied in an interactive visualization and classification tool that supports information retrieval aiming for total recall. Previous studies have already shown that allowing user's interference in classification processes can improve the efficiency of the classifier's convergence (PAIVA et al., 2015).

Similarly, Koch, Heimerl and Ertl (2013) presented a solution that lets a user visually 
and interactively build binary classifiers in order to improve a document retrieval task using multiple views. The classification algorithm used was the linear SVM, where the goal is to show users the most uncertain documents that the classifier found relevant and not relevant for labeling first. Documents are shown in a 2D scatterplot that displays the current state of the classifier. By labeling the documents, the user can see a preview of how the other documents would be classified.

In conclusion, the rationale is to have a default text classification algorithm and, ideally, the user would be able to choose among other algorithms since the classifier accuracy is highly related to the data and domain under analysis (KAMATH; BUKHARI; DENGEL, 2018).

\subsection{Techniques for Text Visualization}

In information retrieval tasks it can be very useful to have an overview of the data, a.k.a, how the corpus is organized. At this point, we know that the documents can be represented as vectors where similar documents should have representations that are close to each other in the vector space. However, these vectors are high-dimensional, which makes impossible for us to visualize them. Dimensional reduction techniques can be helpful in this context, to obtain 2D or $3 \mathrm{D}$ vectors that can be represented in a $2 \mathrm{D}$ visualization, while preserving as much as possible the relationships defined in the original space. Besides visualization tasks, dimensionality reduction also facilitates classification and compression of high-dimensional data.

Since it is beyond our scope to detail such techniques, we rather focus on a few techniques found most relevant to our studies. The first technique is the Principal Component Analysis (PCA) which is a convex and full spectral technique. A convex technique means that the objective function does not contain any local optima and by full spectral it implies that PCA performs an eigendecomposition of a full matrix which, at the end, provides the similarities among the input vectors. Similar to PCA, multidimensional scaling (MDS) is also convex and full spectral (MAATEN; POSTMA; HERIK, 2009).

PCA dates back to 1901 (PEARSON, 1901). It consists in preserving the inner product of data points by applying an orthogonal transformation in order to achieve the best fit when placing the data on a line (or plane). This is done by retrieving the dimensions that maximize the variability in the data, which are called the "Principal Components". Thus, the first principal component will be the one with the largest possible variance and the others principal components will have the next highest variance, while being orthogonal to the other components. In order to find the dimensions that offer maximum variability, a common approach is to compute the covariance matrix which shows the linear tendency between each pair of data points. The principal components will be given by the eigenvectors associated with the largest eigenvalues of the covariance matrix.

The MDS technique (TORGERSON, 1952) consists in three steps: (1) Find the com- 
parative pairwise distances among data points (distance - a unknown constant), (2) find an approximation for the unknown constant and (3) place the data in the space according to the absolute distances found in the previous step. This unknown constant is estimated as the value which added up to the distance will allow the data to be fitted by a real Euclidean space with the smallest dimensionality possible. The classical multidimensional scalling is one way of implementing MDS. A popular MDS approach is the Force-Directed Placement (FDP) (FRUCHTERMAN; REINGOLD, 1991) which creates forces for every pair of data points and the system reaches the equilibrium by pushing and pulling them out. This technique is considered equivalent to MDS when the spring forces are proportional to the dissimilarity of points in both the real and the projected space.

As stated by Maaten, Postma and Herik (2009), PCA and MDS face two major drawbacks. First, PCA does not scale to very high-dimensional data, since the covariance matrix is proportional to the dimensionality of the data points. However, this issue might be solved by MDS if the number of data points is lower than the their dimensionality since classical scaling scales with the number of data points. Another drawback is that both techniques focus on maximizing the global pairwise distance among the data points and do not prioritize the similarity of neighboring points, which may be more important.

The results from Maaten, Postma and Herik (2009) suggested at the time that most nonlinear techniques do not outperform PCA on natural datasets, even though they are capable of learning the structure of complex, nonlinear data. Besides, when the number of data points is greater than the number of dimensions, nonlinear techniques have a higher computational cost than PCA. Even though convex techniques such as PCA are popular, the author suggests that finding a sub optimal objective function is more viable than optimizing a convex objective function due to its complexity to optimization. This is supported by the results obtained from the t-SNE technique which is a non-convex MDS variant that was not yet available when the comparative study was performed.

The (t-Distributed Stochastic Neighbor Embedding) t-SNE technique was proposed by Maaten and Hinton (2008) for data visualization. This means that the goal is to give each data element a position in a $2 \mathrm{D}$ or $3 \mathrm{D}$ space. As opposed to linear techniques such as PCA and MDS, that focus on keeping dissimilar data points far apart, non-linear methods such as t-SNE usually focus on keeping similar data points close together, which is typically impossible when using linear techniques. As the name suggests, t-SNE was derived from the Stochastic Neighbor Embedding (HINTON; ROWEIS, 2002), a technique to embed high-dimensional data into a low-dimensional space in a way that reduces the agglomeration of points in the center of the map, thus improving the quality of visualizations.

Maaten and Hinton (2008) modified the SNE's cost function, which is difficult to optimize and generates the agglomeration problem. Thus, t-SNE applies a symetrized version of the SNE cost function and uses the Student-t distribution, rather than a Gaussian, for computing similarity 
between two points in the low-dimensional space, which speeds up calculations. Also, t-SNE applies a heavy-tailed distribution of the points in order to alleviate the two problems mentioned above.

For the tests, the author first applied PCA to reduce data dimensionality, which speeded up the computations and reduced the noise. After comparing t-SNE with other seven dimentionality reduction techniques, including Samon mapping, Isomap and Hessian Locally-Linear Embedding (Hessian LLE) on five data sets from different domains, results have shown the superiority of t-SNE projections compared to other methods (Figure 5).

Figure 5 - Projections using different dimensionality reduction techniques of 6000 digits from the MNIST dataset

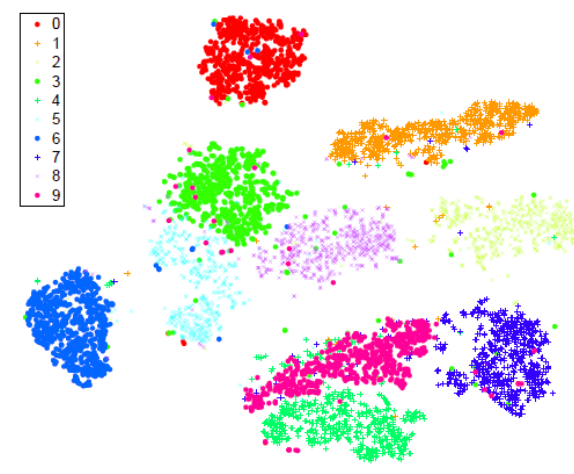

(a) t-SNE

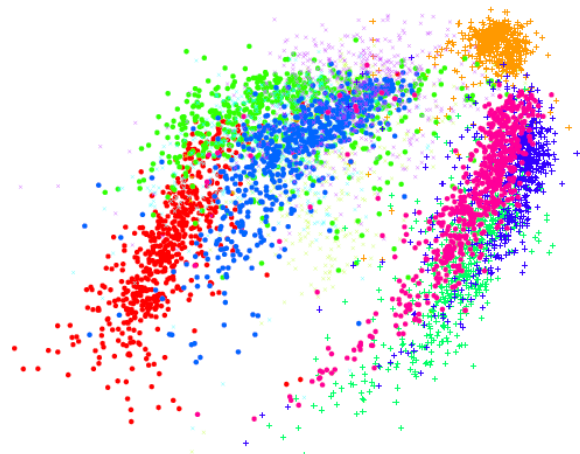

(c) Isomap

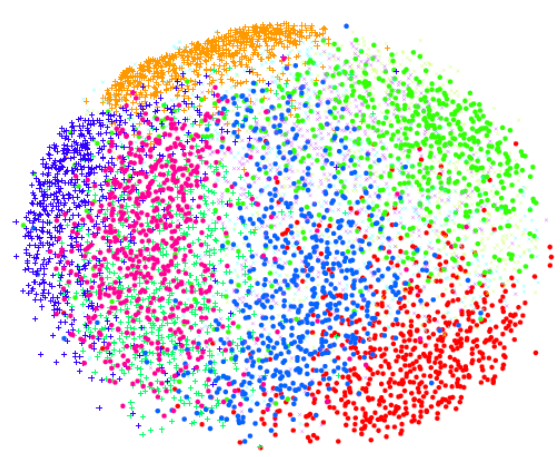

(b) Sammon Mapping

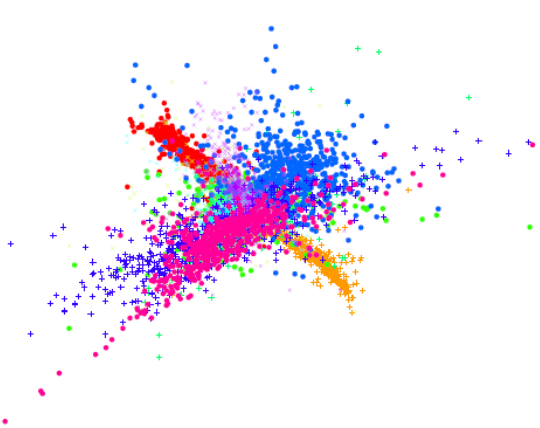

(d) LLE

Source: Maaten and Hinton (2008)

Since t-SNE has quadratic complexity, it fails to scale to many data points $(10,000$ or more). However, showing so many points at once in a visualization can be overwhelming and usually is not desirable. Other ways to mitigate this problem include modifying t-SNE to display a random subset of data points (named landmark points), using modified versions of t-SNE such (MAATEN, 2014b; INGRAM; MUNZNER, 2014; PEZZOTTI et al., 2016), or using other approaches that allow adding new points in the space as needed, such as the Least Square Projection (LSP) (PAULOVICH et al., 2008) or that have a hierarchical structure, such as the 
Hierarchical Point Placement Projection (HiPP) (PAULOVICH; MINGHIM, 2008).

The LSP technique relies on selecting control points representative of the data distribution. These points are mapped in the 2D space using MDS. Then, the algorithm creates a linear system in order to map the other points using their similarity information and the Cartesian coordinates of the control points. That way, the system makes it possible the simple addition of new points, since it only interpolates the new points while aiming at preserving neighborhood relations. LSP yields high precision and fast computation specially in nonlinear sparse spaces such as high-dimensional document representations. The projection can be generated from the distance relationship matrix or from attribute relationships of a vector representation.

HiPP is a variation that focuses on allowing exploration through different levels of detail. The projection first shows clusters of the data and details are provided on demand by expanding the clusters. Thus, instead of dealing with approximations, it splits the data into clusters and places them in the display according to the cluster's similarity, which is way faster than calculating the similarities among all the datapoints. By optimizing the use of space, the technique solves the problem of point overlapping typical of t-SNE and LSP. After constructing the tree, where the leaves represent individual data instances, the projection is done in two main steps: first, the LSP (or other point placement strategy) is used to project the nodes. Second, the distance among points is improved in order to resolve overlaps. Views of data mapped by both HiPP and LSP are shown in Figure 6.

Figure 6 - Projection of LSP and HiPP of 30000 data points from a news articles dataset

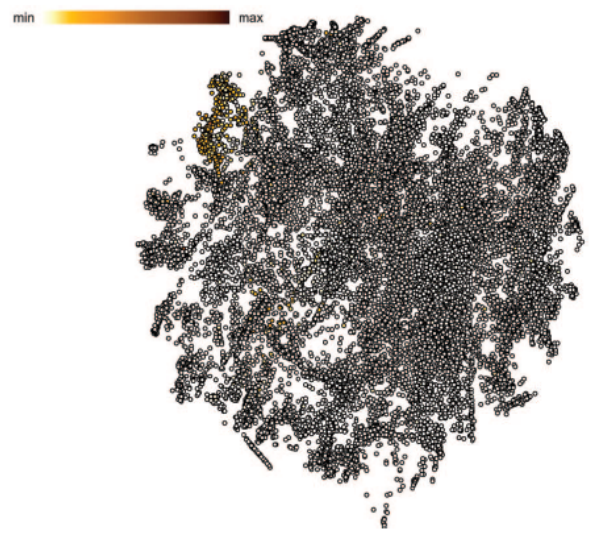

(a) Least-Square Projection (LSP)

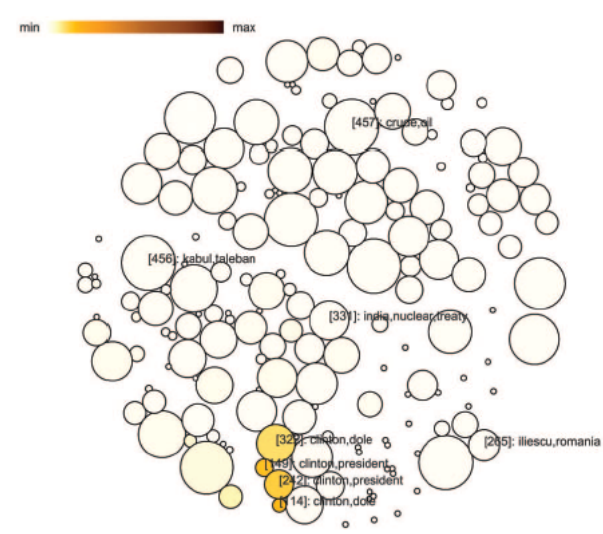

(b) Hierarchical Point-Placement (HiPP)

Source: Paulovich and Minghim (2008)

A drawback of non-linear techniques is that their behavior depends on the chosen parameters, which may depend on the data. For example changing the perplexity and step parameters in t-SNE can yield different results ${ }^{3}$. Similarly, the choice of the control points in LSP interferes in the final result. Nevertheless, according to Sedlmair, Munzner and Tory (2013), 
a good approach is to vary the dimensionality reduction technique to investigate the best option, since there is not a single technique that is best than the others in all scenarios. 
CHAPTER

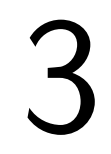

\section{INFORMATION VISUALIZATION FOR INTERACTIVE TEXT RETRIEVAL}

This chapter presents a literature review on text visualizations proposed for use in connection with document retrieval tasks. The studies were organized into three groups: (1) Those that help a user to understand the search result and, thus formulate an ideal query, (2) Those that help a user to understand the corpus and, (3) Those that merge both previous goals. The later is more related to this work, however, both groups 1 and 2 present solutions for their respective goals that were considered relevant to our research.

\subsection{Understanding the search}

Systems that relate text documents to queries, usually represented as keywords of interest, are very relevant to the task of information retrieval specially because they render the search process more transparent than classic search engines, where the user inputs some keywords and the result is a ranked list of documents. When the user understands why a document was ranked high or low, s/he can improve the initial query and thus, increase recall. This is specially important when the user is conducting exploratory search or the ideal query is very complex to formulate.

The VIBE system by Olsen et al. (1993) is one of the first to support document retrieval by displaying the relationship between the documents and the query (Figure 7). It is a prototype system for visualizing a document collection that relies on facet queries. The authors introduced the term "point of interest" (POI), where each point (i.e. document) is located at a unique position in the display as an icon and each POI represents a set of user-defined keywords serving as a query. Therefore, the system allows a user to perform multiple queries simultaneously, each one represented by a POI which enables analyzing a document from different perspectives.

The investigation is possible because documents are placed on the display according 
Figure 7 - The VIBE system. Documents represented as rectangles are placed according to the keywords or POIs they contain.

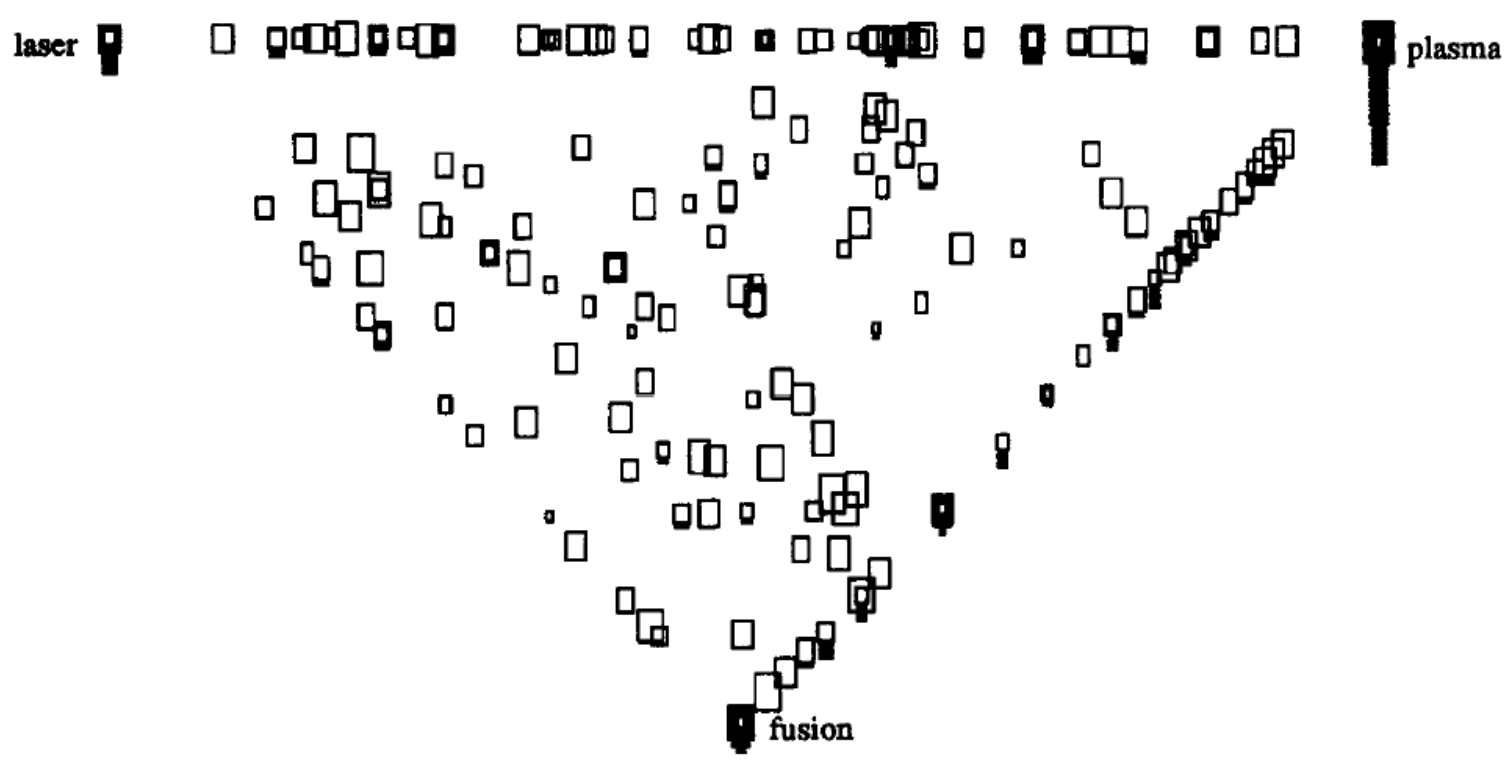

Source: Olsen et al. (1993)

to their similarity to each POI. For example, a document that refers to a single POI will be placed at the same location as the POI's icon. On the other hand, if a document refers to multiple POIs it will be placed between them (the exact position will depend on the similarity among the POIs). The scoring algorithm which determines the relationship between a document and a POI is defined by the user and could be the count of terms from each POI.

The user can click on a document to view its content and, if multiple documents are placed at the same position on the display, the visualization shows lines under the document icon, where each line represents another document that can be accessed by multiple clicks on the icon. Moreover, users can reposition the POIs, change their definition by modifying weights of POIs keywords and ignore a certain POI. The visualization does not allow many interactions and it does not scale to many points of interest since it would be difficult to identify the topics from documents in the center. However, the idea of points of interest is used in many following works.

Similar to the VIBE system, the Visual re-ranking system (KLOUCHE et al., 2017) allows a user to input keywords and a 2D visualization with both the keywords and their related documents are spatially placed according to their similarity (Figure 8). The user can activate or deactivate a keyword in order to see how the documents are rearranged. A major difference relative to the VIBE system, however, is that it also shows a ranked list of documents and the user can re-rank this list by selecting a position in the visualization. For example, if the user selects a position close to a specific keyword, the ranked list will be updated to show documents more related to the selected keyword.

Another solution to understand the result of a search while prioritizing retrieval of long 
Figure 8 - The Visual re-ranking visualization. Red rectangles represent the keywords of interest, gray rectangles are deactivated keywords, the blue icon is the exploration cursor that will define the location to which the algorithm should use as reference to re-rank the list in (2). The circles in red in (1) represent the documents that are currently being displayed in the ranked list in (2). The circle sizes encode the overall relevance of the document to all the queries phrases.

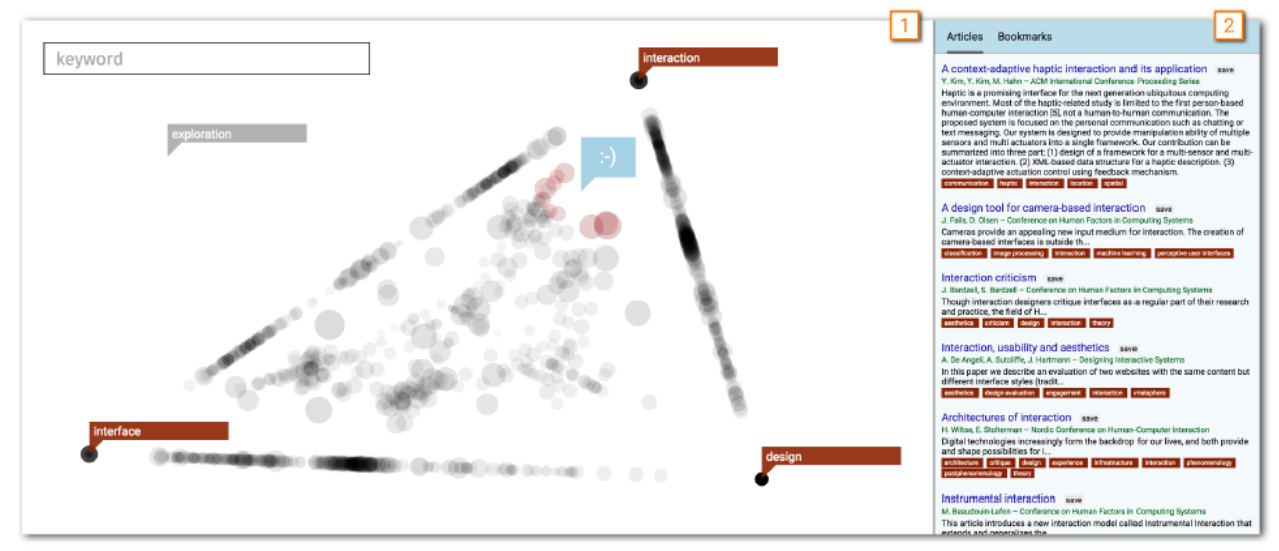

Source: Klouche et al. (2017)

texts is the Tile Bars system (Figure 9) (HEARST, 1995). It shows not only document relevance but also why a specific document received a high ranking. To achieve this goal, the system presents additional information such as term frequency, term distribution in the text and term overlapping within the document.

TileBars relies on facet queries as the VIBE system, in which users can input topics or queries. It is possible to explore parts of interest in a document by means of a visualization that shows a list of large rectangles representing a document. Within each rectangle there are small squares (or tiles) color coded from white to black, where the darker the tile, the more frequent is the corresponding term in a specific part of the document. The difference between VIBE and TileBars is that the former does not address the issues of term distribution, frequency and overlap. Moreover, TileBars would be helpful to determine whether a search was useful and which additional information could yield better results. An user can perform multiple queries and explore the results in order to improve the original query.

A problem noticed on the TileBars system is that once a user opens a document on the list, s/he loses the terms' locations. The study by Byrd (1999) uses the scrollbar feature to code term information in each document. This is accomplished by positioning small colored icons overlapping the scrollbar, where each color represents a term and its variants. The system helps users to quickly find parts of interest in a document and decide on its relevance. A limitation is that it does not scale well with the number of terms, since distinguishing more than roughly five colors is difficult.

Similarly, the SmartSkim (HARPER; COULTHARD; YIXING, 2002) system presents a histogram visualization representing a target document where each bar corresponds to a fixed length section of the text. The height of each bar represents the importance of that section to the 
Figure 9 - The TileBars system. The user can type in multiple queries and a list of documents that contain the queries' terms is presented. Each item of the list contains a visualization that shows the portions of the text that are relevant to the queries where higher the saturation, more relevant that part of the text is to the search.

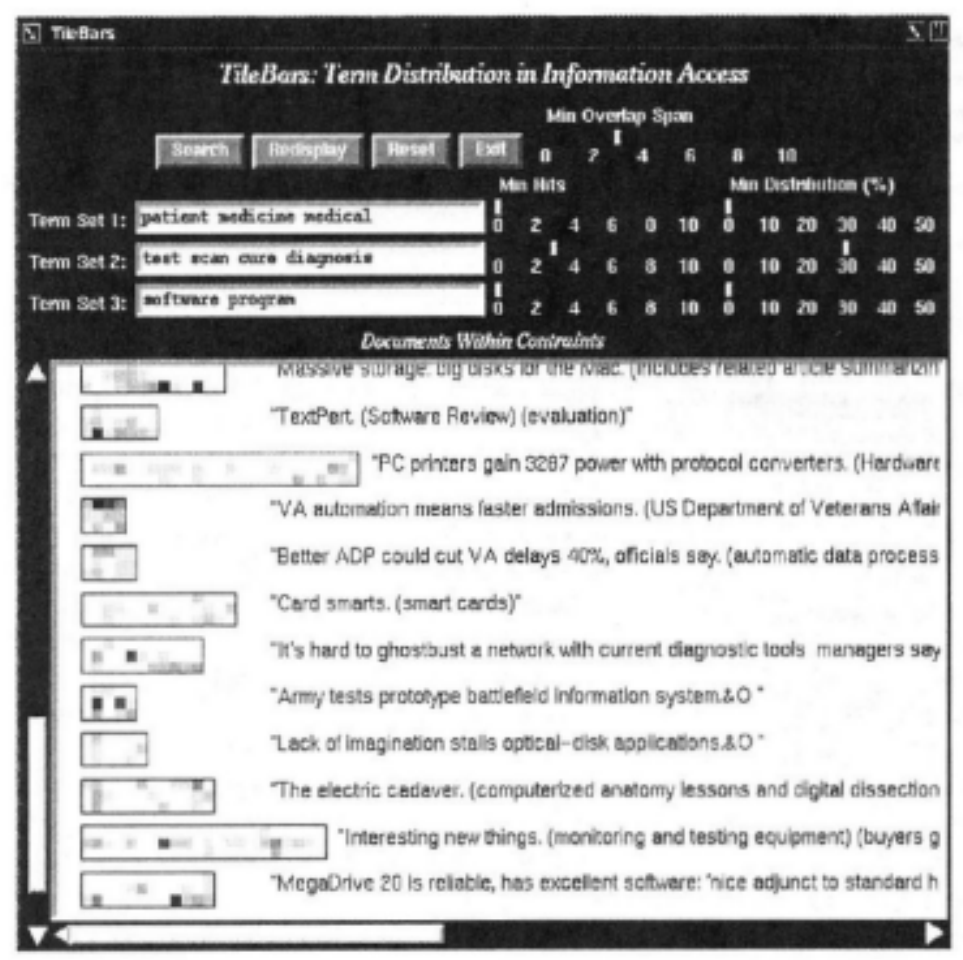

Source: Hearst (1995)

query. By clicking on a bar, a document view is shown with the section selected centred and the query terms highlighted.

Sphere or circular visualizations are also used to depict the relationship of the query to the search results. A solar system metaphor is applied in the WebSearchViz visualization system, where the query is the central point (as the sun) and the subjects or topics of interest retrieved from the query along with the web search results are scattered around it as the planets and asteroids (see Figure 10). Also when the user moves a subject around the circle, the web pages related to the subject also rotate and their rotation speed is defined based on the semantic strength to the subject. In order to give more or less importance to specific parts of a subject, the user can set weights to keywords that were extracted from the search results. The subjects are dynamic and the user can add, delete or modify them at any time (NGUYEN; ZHANG, 2006).

Showing how the search result relates to the query helps a user to understand the search process, however, it is not always enough. There are situations a user needs more assistance to create an initial query or to refine it, specially when formulating an ideal query is complex or when the user does not know exactly what s/he is looking for. For this reason, some studies offer more tools for query creation and refinement. Certain systems assist users in refining an initial query by letting them explore the corpus, offering resources to reduce the human effort 
Figure 10 - The WebSearchViz system. In (a) the user can see three subjects of interest (data visualization, software visualization and website visualization). The query is located at the center of the circle and the resulted web pages are the pink squares around it. In (b) the user sees how the results are related to a subject when s/he moves the "website visualization" subject around. It is clear that the documents in (1) are more related to "web visualization" than the documents in (2) since they barely moved.

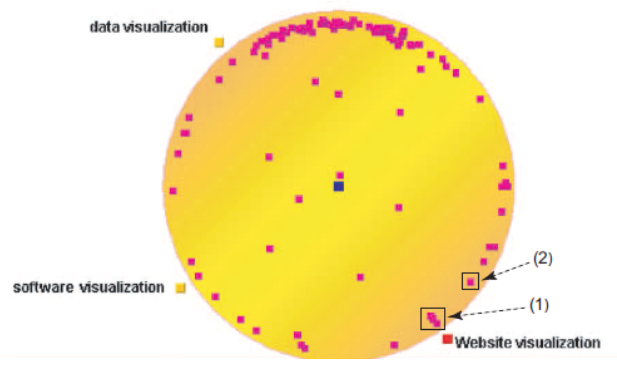

(a)

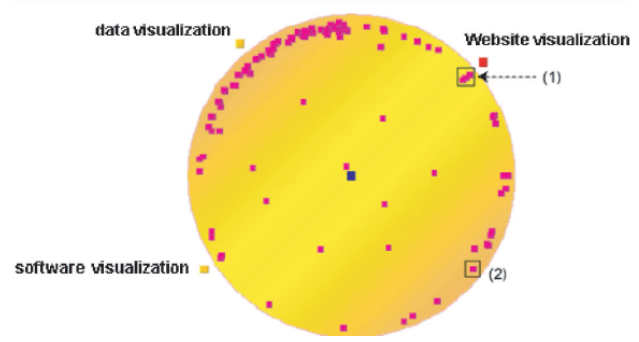

(b)

Source: Nguyen and Zhang (2006)

and expertise required. They typically resort to the metadata associated with specific document categories, such as news articles or scientific papers. The documents and/or the terms are usually organized into groups to facilitate user navigation. Clustering algorithms can be employed to organize the documents according to topics they have in common.

One of the early attempts to allow users to refine a query is the Scatter Gather visualization (CUTTING et al., 1992), illustrated in Figure 11. The authors use clusters to help users defining a query, rather than exploring the corpus. In this way, it is not necessary to provide initial terms or query. By selecting the initial cluster, the algorithm creates new clusters on the fly. The visualization does not provide many interactions besides clicking on each cluster to reveal new clusters underneath.

The WebRat visualization (GRANITZER et al., 2003) is another solution that allows a user to refine an initial query by clustering the results. The user first enters the initial query then the system returns the results from different search engines grouped into clusters labeled by the important topics of each cluster. The user can refine the query by adding a topic to the search (Figure 12). Results have shown that the system can be very useful to users with limited knowledge on the search domain.

Similarly, VisElabor (KORHONEN; LITOLA; TARHIO, 2007) consists of a visualization with four main views in order to let the user refine an initial query applied to a search engine. The views include a list view returned from the search engine, a category view which is the result of a cluster algorithm to categorize the results, a graph view showing the relationship among documents and a full text view that presents the documents to the user. By manipulating these views, a user can submit new queries through the system and it increments the current visualization with the new results, thus improving the initial query. 
Figure 11 - The Scatter/Gather visualization. The user is presented with an overall view of the important terms in the collection where s/he can choose some terms in order to filter the visualization to show new terms related to the selected ones.

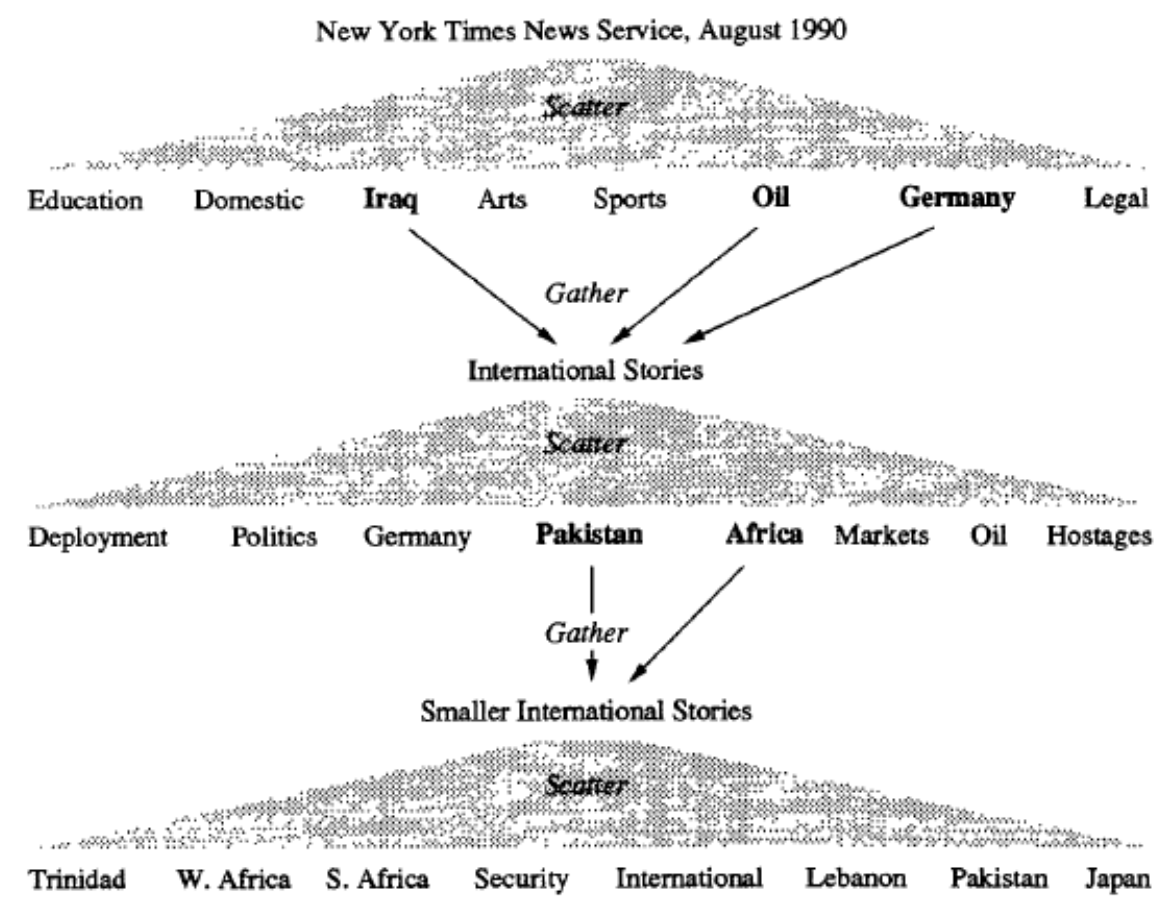

Source: Cutting et al. (1992)

The system STORIES in time (BERENDT; SUBASIC, 2009), illustrated in Figure 13, downloads news articles from the web and presents a graphical visualization to support story understanding and search. For this goal, it extracts topics from a time-indexed textual document and displays a sequence of story graphs in order to convey the story evolution. As users search on nodes of a subgraph, the retrieval is restricted to documents relevant to those subgraphs, expanding the original query. Users can specify a date, select a story to track, zoom in or out of a story stage, get the text overview and more.

Cite2vec (BERGER; MCDONOUGH; SEVERSKY, 2017) is a visualization tool for exploring and browsing documents based on how they are referenced by others. Words that represent the content of the document collection are projected and the closeness of a document to a keyword depicts its manner of usage via citation contexts, while maintaining the relationship among other documents. The documents are represented in a scatterplot and the approach is capable of handling 20,000 to 30,000 documents. A word2vec model extension was employed in order to project both words and documents in the same space.

The user can search for one or more keywords or phrases in order to explore the visualization, as illustrated in Figure 14, where the user searched for "random forests" and "depth", which results in papers containing a composition of these phrases. However, a limitation is that recently published papers might not have sufficient citations to be plotted into the visualization, 
Figure 12 - The WebRat visualization. On the left is presented the results for the query "Knowledge Management". On the top right, the result after the user zoomed in into the keyword "certification" is shown and on the bottom right the user is refining the query with the word "information".

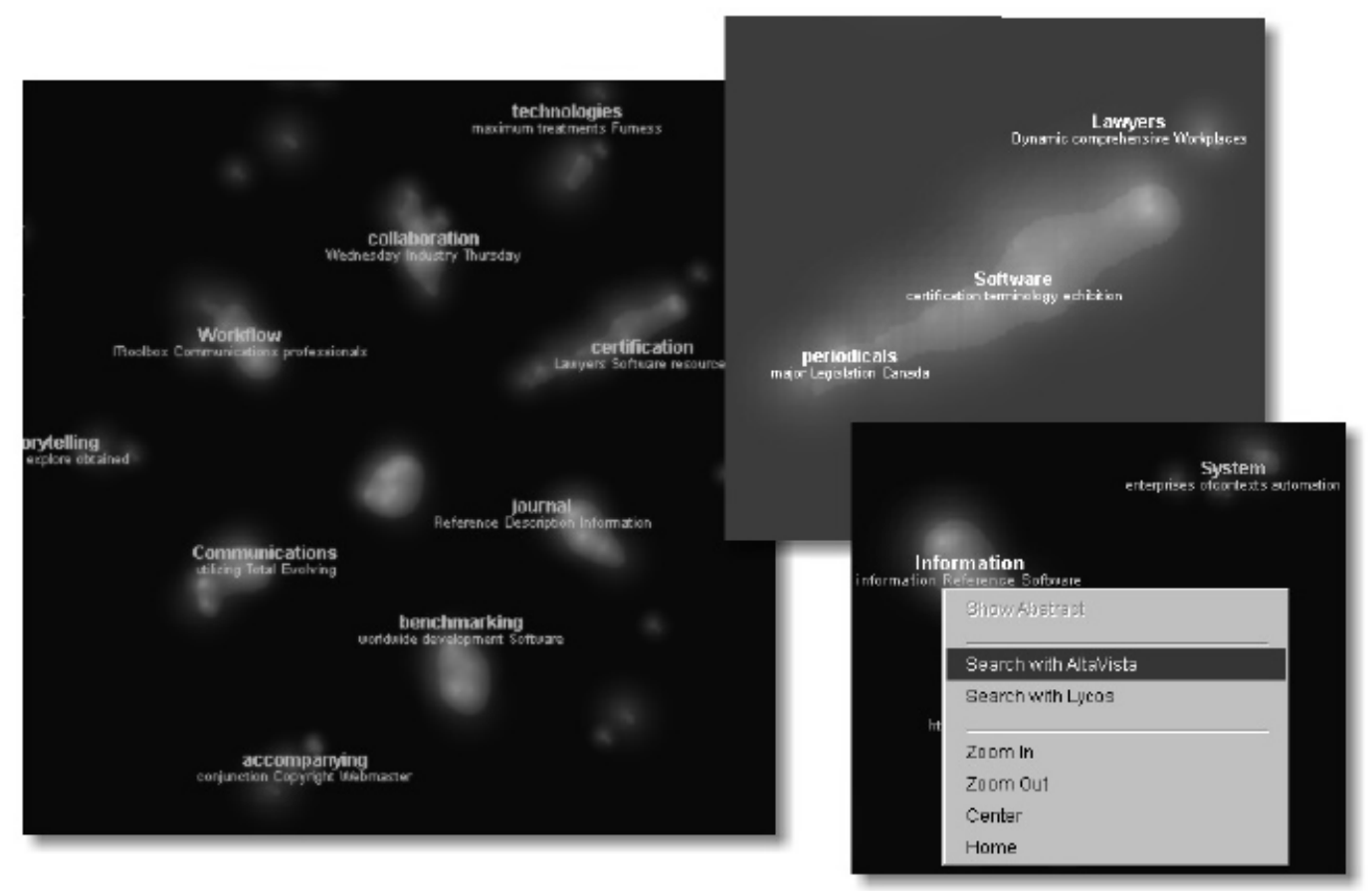

Source: Granitzer et al. (2003)

even though they could be relevant to the user. Since the visualization focuses on how an article is being used, if a user wants to search for an author, only the papers that explicitly cited that author are shown.

As discussed in Chapter 2, vocabulary mismatch is an additional issue in document retrieval, when different terms refer to the same concept, or occurrences of a single term indicate different meanings. It can be handled, e.g., by assigning a similar distributional vector to words that appear in the same context in a corpus, i.e., surrounded by the same words. Zimmer et al. (ZIMMER; SAHLGREN; KERREN, 2017) introduce a system that tackles vocabulary mismatch in the context of scientific papers, reinforcing that articles may address related topics even if they use different terms to describe similar concepts (ZIMMER; SAHLGREN; KERREN, 2017). Authors use a corpus of scientific papers to create a word similarity matrix. The visualization uses a collection of 2,752 documents as example and consists of three graph views. One network represents the papers as nodes connected considering their citations, the second network maps the author and co-author relationships and the third network consists of all the author's affiliations and edges are added if authors from different affiliations publish a paper together.

The authors also used both bag-of-words and TF-IDF techniques in order to find papers that include a certain keyword and its importance in the text. To find synonymous terms, a word similarity matrix was created. The rationale is that words that were used in the same context 
Figure 13 - The STORIES system. The main view shows a story graph of news regarding Britney Spears in 2007. The main window on the center-left represents the first story graphs resulted from the search and, as a user narrows the time window (center-right), it is possible to gain new insights about the documents since new nodes are added. If the user selects edges between the nodes, a list of pertinent documents to those nodes is presented in the center-bottom where the text can be inspected in the left.

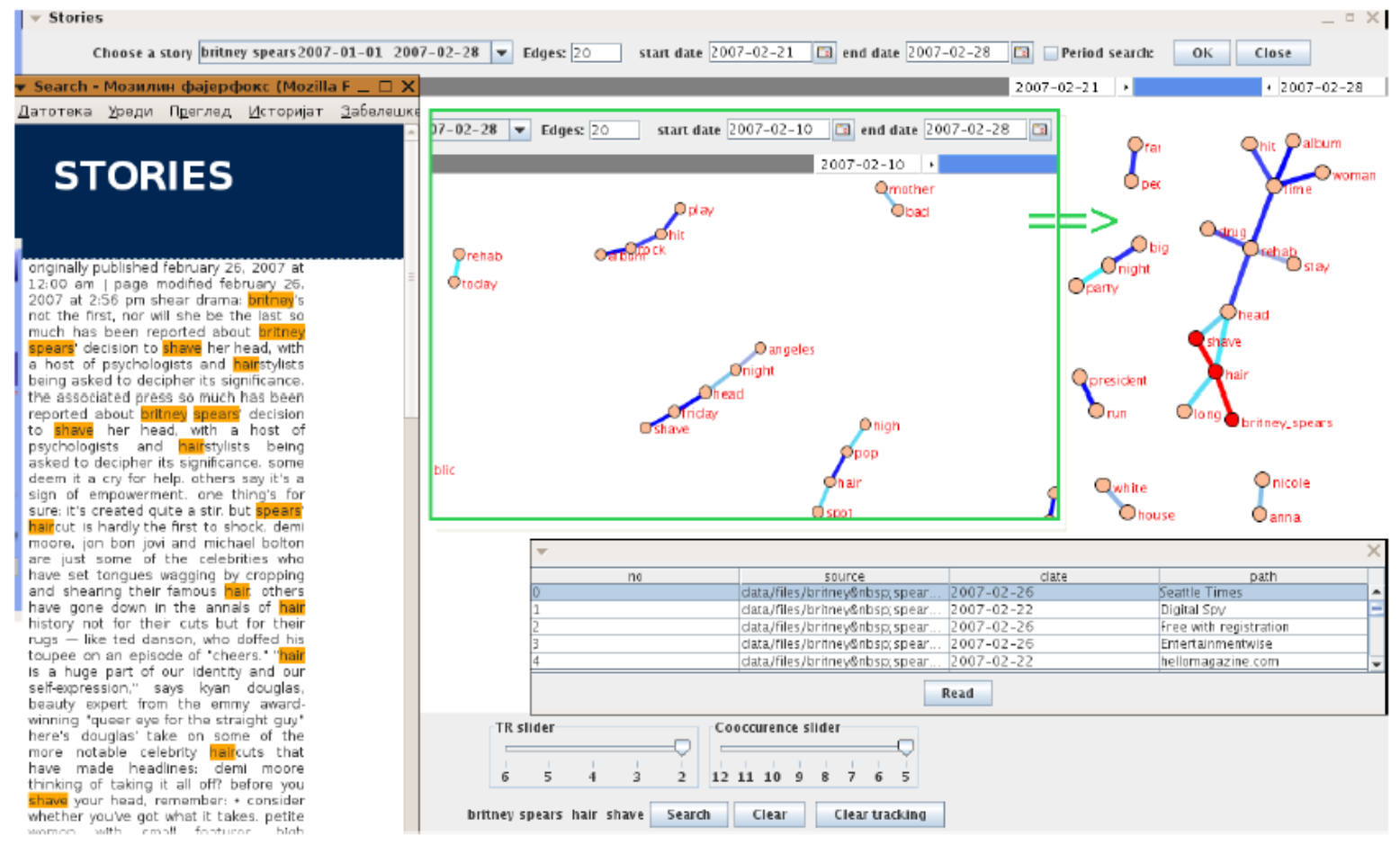

Source: Berendt and Subasic (2009)

(surrounded by the same words) receive a similar distributional vector which is calculated using the random indexing (RI) that is based on an incremental random projection of a count-based model. The cosine similarity metric was used to generate the word similarity matrix.

The initial visualization does not show any graphs but the bag-of-words and TF-IDF frequency of the entire collection. Thus the user can assess how to start a search and add or remove nodes on demand. The user can search on a network and the visualization creates links with the two other graphs on mouse hover. By right-clicking a node, it is possible to see the bag-of-words and TF-IDF frequencies of that document. By using three different graphs in the same view, one might need some training to deal with the amount of information. The system scales for up to 10.000 nodes and edges since it uses WebGL instead of SVG-based node link visualization approaches.

The aforementioned systems rely mostly on the user knowledge, whereas classic IR systems usually suggest new documents that might be relevant. There are visualization systems aimed at assisting users to perform IR tasks with high recall, while offering personalized recommendations, such as the Visual Information Retrieval and Recommendation system (VisIRR) (CHOO et al., 2018). VisIRR has been created to support large-scale document discov- 
Figure 14 - The cit2vec system. A search for depth-based random forests techniques shows techniques and data used by documents in this domain.

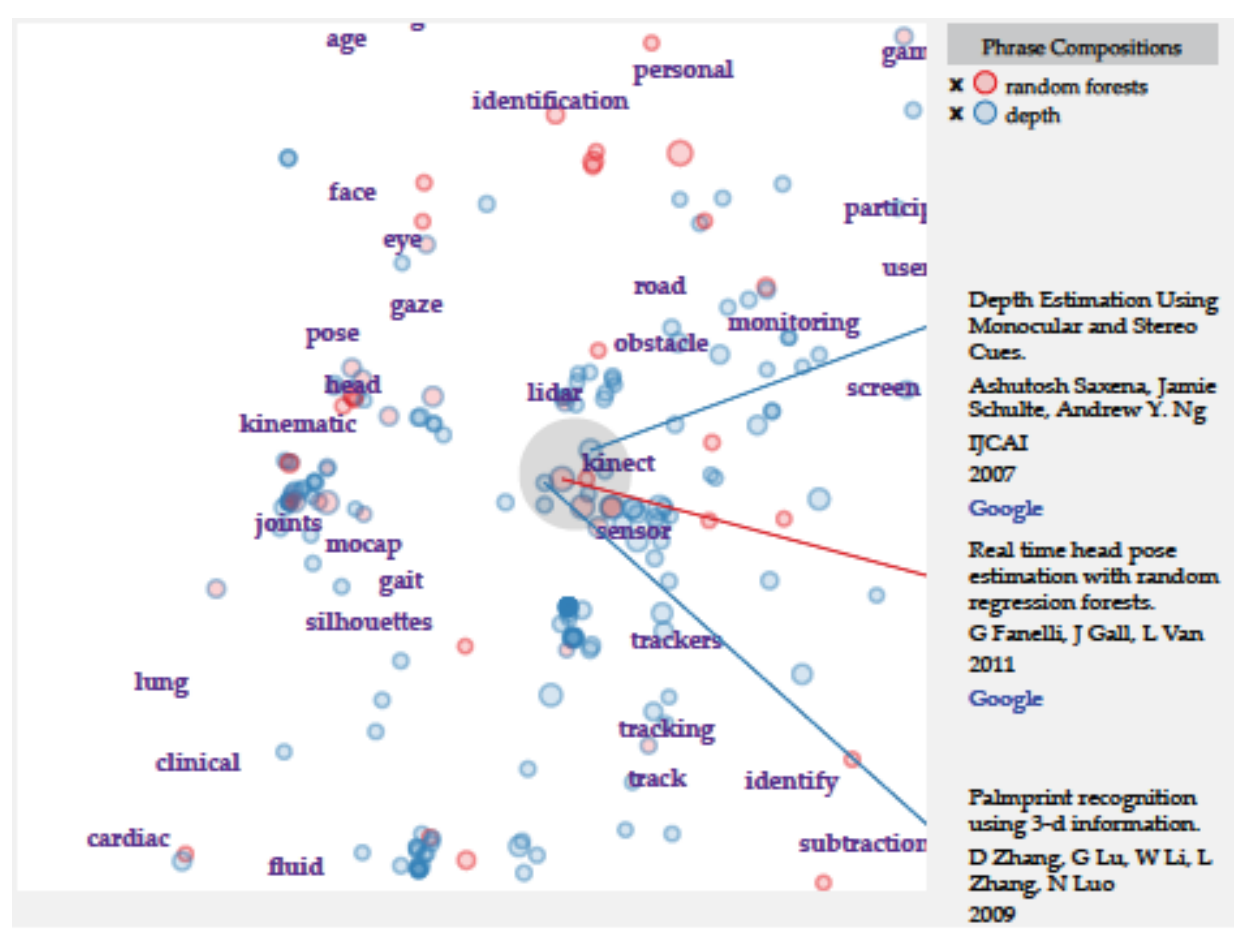

Source: Berger, McDonough and Seversky (2017)

ery and utilizes a publication database with nearly 430,000 academic research articles. It relies on document metadata to offer recommendations based on the documents' ratings or number of citations. The system uses topic modeling in order to retrieve the most important topics from a document corpus and then groups similar topics together. Afterwards, the documents are projected into a 2D scatterplot. The starting point of the system (see Figure 15) is an overview visualization of part of documents retrieved by the user query.

By hovering a circle in the scatterplot the user can explore the document's details. Besides, there is a list on the bottom of all the documents sorted by the number of citations. By zooming in, the user enlarges a particular topic clusters revealing more specific topics to that area. The user can also apply additional queries and compare between the new and the previous visualization results. In order to help the system to recommend relevant papers, the user can assign ratings to documents. Another way is to change the recommendation type to citation-based which shows the highly cited documents that are similar to the rated document. The system shows a lot of possibilities and efficiently helps the user to find new documents and improve the initial query. However, it currently relies heavily on citation and co-authorship information.

In order to help the user to refine a query, some systems rely on visualizations that show how the documents in the search result set are related, where similar documents are spatially placed close to each other. The reason is that in order to give the user the ability to improve or create a query, a system needs to guide users with insights and possibly recommendations. Based 
Figure 15 - The VisIRR system. The user can type a query in A, see the results in both the scatterplot and in the tabular view in B, see the list of recommended documents in C, zoom-in to get more details (D) and visualize the topic cluster summary in E.

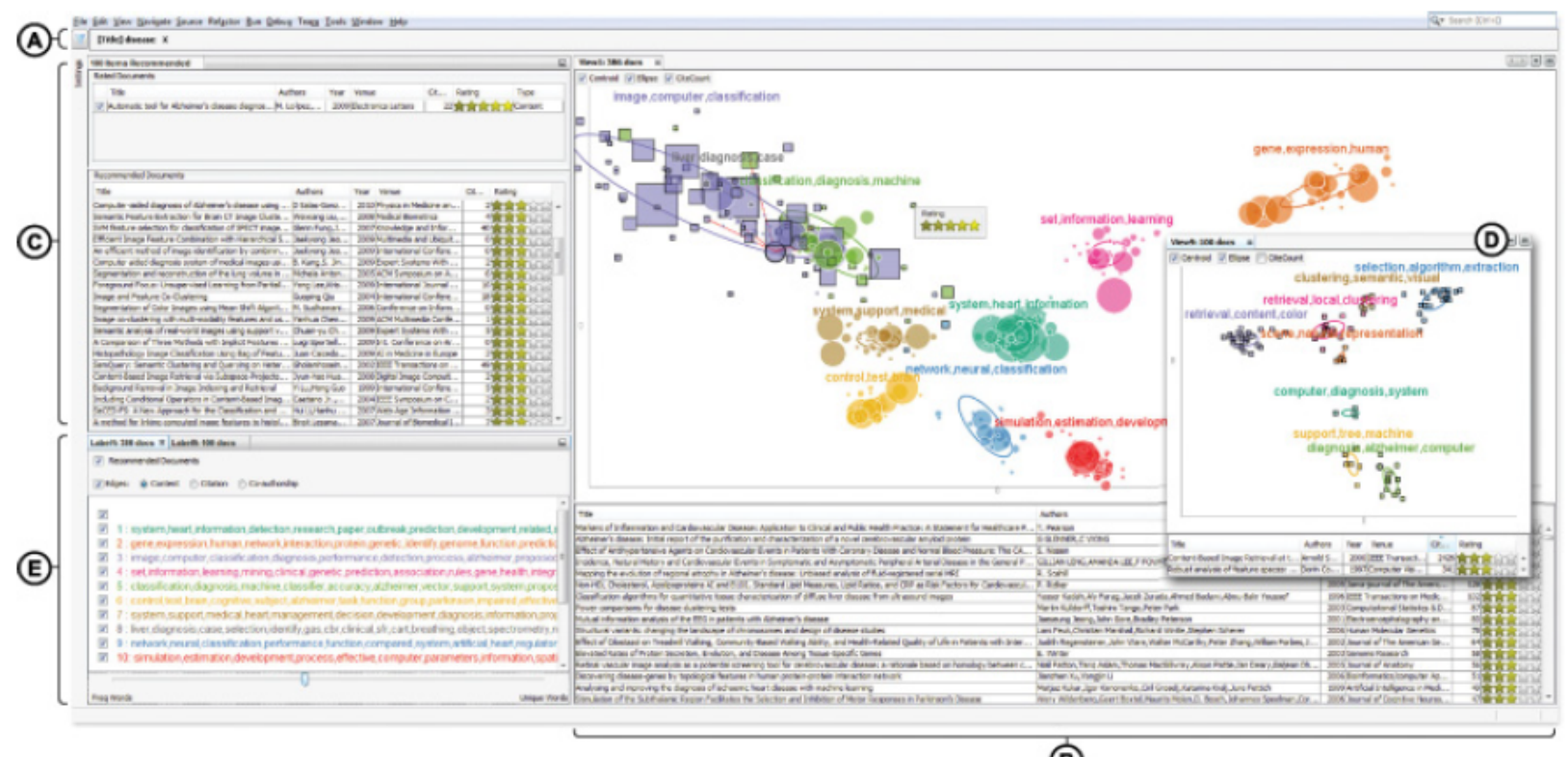

(B)

Source: Choo et al. (2018)

on the studied solutions, a technique that is usually adopted is to cluster documents by similarity, usually based on relevant keywords, where the k-means clustering is very popular for this task.

\subsection{Understanding the corpus}

One of the goals of this project is to show how the documents retrieved from a query are related. The following solutions also address this problem, however, their main focus is on the exploratory search of a document corpus in order to give users new insights, and not specifically on improving an initial query nor retrieving a set of relevant documents. Besides, most contributions consider specific types of documents, such as news articles and scientific papers with specific metadata associated that can enhance the visualizations. This is not the case of the visualization solution described in this dissertation, since it should handle different domains.

A classic set of visualizations derived from the IN-SPIRE project includes contributions such as the Galaxy of News visualization (RENNISON, 1994) where the main view is shown in Figure 16, in which the goal is to give users a broad understanding of a news database introducing the visualization of themes. The software relies on an Associative Relation Network (ARN) to establish relationships among news articles, where the affinity between symbols contained in an ARN defines weighted relationships between documents. The process consists of extracting key 
information, such as keywords, from a news corpus and insert the key symbols into the ARN. Then, the system searches the network to extract hierarchical relationships between the articles and render the nodes as words in the display, in agreement with their similarity. Since time complexity is a concern when dealing with relational graph representations, the relationships among articles are computed offline and outside the front-end application. Users can zoom in into a specific area and the articles will be reorganized according to the new point of interest. It is also possible to read an entire document.

Figure 16 - The Galaxy of News visualization. Documents are shown as stars in the sky. Symbols such as keywords, locations, time event(s) occurred, subjects, and actions are extracted from news articles and fed into the ARN.

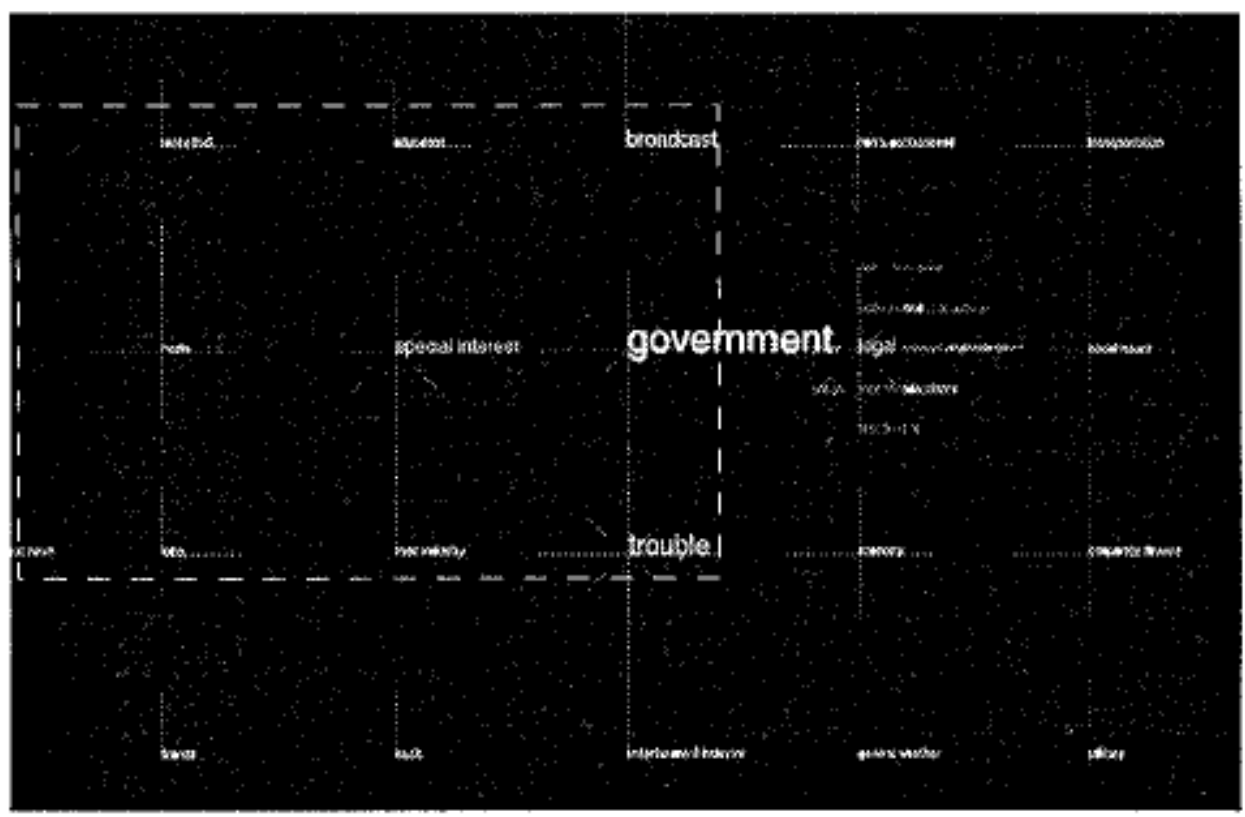

Source: Rennison (1994)

Retrieving the corpus in a different process speeds up the calculations thus providing a smoother user experience within the visualization. Nevertheless, there is the concern of keeping the corpus up to date. The Galaxy of News system served as inspiration to other projects such as the ThemeScape visualization developed by Wise et al. (1995) which complements the Galaxy system by showing landscapes rather than points in the space. This gives users a better idea of the semantics behind a group of documents. Also, the Typograph (ENDERT et al., 2013) visualization shows terms, phrases, snippets and full documents according to their similarity allowing a user to identify clusters and investigate the space by zooming into specific regions. Additionally, an interesting feature of this visualization is that a user can see different contexts in which the query is relevant (see Figure 17).

While the previous solutions handle corpora of news articles, the following solutions are concerned with showing relationships among scientific papers. This category usually deals with typical metadata of this type of document, such as references, citation, author or venues. In the 
Figure 17 - The Typograph system. The user entered the query "sun" and some phrases such as "microsoft windows" and "single released" show different contexts that the query was utilized. The user can interact further in order to visualize snippets showing the use of the query within the text.

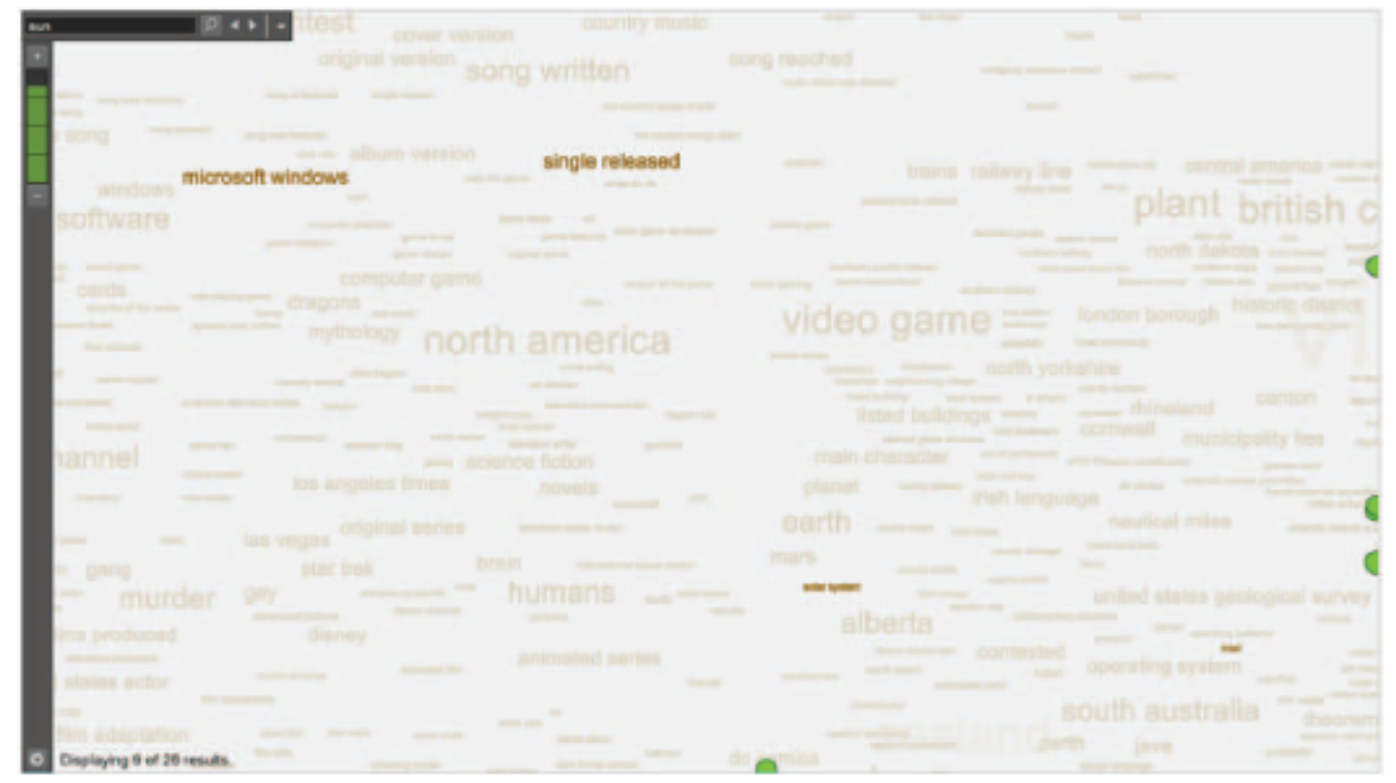

Source: Endert et al. (2013)

BEAD system presented by Chalmers and Chitson (1992) scientific articles are considered as particles and are placed close to each other based on physical forces that attract similar articles. This approach creates clusters (metaparticles) of documents in a way that a cluster is represented by a single term vector which accelerates the insertion of a new document into a specific cluster.

The BEAD visualization draws nodes as IDs (Figure 18), and the color intensity of nodes represents the document's distance from the query. It is possible to zoom in a chosen particle to see more details and adjust the radius which defines the sphere of interest. The system does not allow many interactions such as filtering or selection of articles and provides a cluttered view of numbers instead of titles, which makes the exploration difficult.

The CiteSpace II system (CHEN, 2006) illustrated in Figure 19 considers the time attribute and provides a generic solution to support the identification and visualization of emerging trends and abrupt changes over time in a collection of scientific papers. It uses a heterogeneous network where clusters are defined based on identifiers of groups of articles. Besides, the visualization uses a time-zone view to depict events over time. A user can choose the domain and alter parameters within the visualization. By grouping nodes in a network, the user can interpret the collection and thus perform a more efficient search.

Even though the contribution by Felizardo et al. (2012), illustrated in Figure 20, focuses on analyzing scientific papers, it differs from the others in several aspects. Users can add and remove documents from the visualization. It relies on Visual Text Mining (VTM) to enhance systematic review procedures, in which the task is to review a list of retrieved papers in order 
Figure 18 - The BEAD visualization. Documents are represented by their IDs and the distance from the query is mapped as the color intensity of nodes.

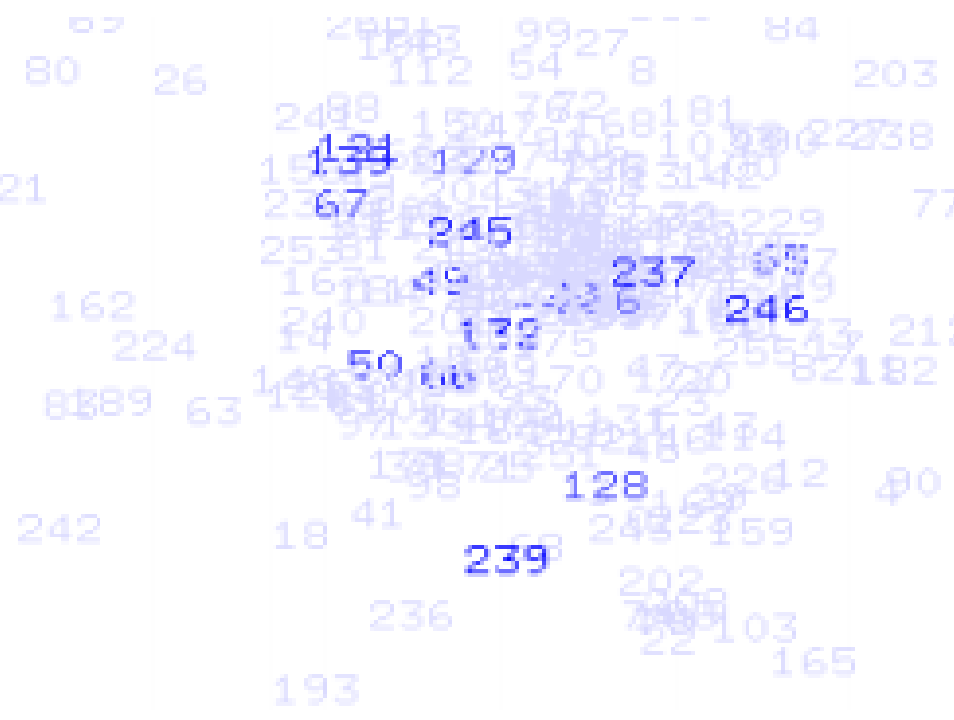

Source:Chalmers and Chitson (1992)

to prevent the exclusion of relevant studies from a survey. Their visualization is split into two views, namely a content map and a citation map. The first shows document content similarities and dissimilarities and the second shows how documents are related based on direct citations or cross-citations.

Both views rely on a graph visualization where nodes that represent documents are placed with a force-based strategy. Nodes can have three different categories (added, removed or referenced) and the user can explore the network in order to improve his/her literature review. Results indicate that the tool yields a higher performance than manual selection, with similar accuracy of a traditional selection review task. Similarly, the solution from Chou and Yang (2011) called PaperVis, also focuses on scientific papers in order to facilitate the literature review tasks.

The Action Science Explorer (ASE) (DUNNE et al., 2012) is a prototype system aimed at allowing users to select key papers, explore citation patterns and identify clusters. The main visualization is a node-link diagram that uses a force-directed layout and shows papers as nodes connected by their citations. An interesting aspect of this visualization is that it shows the context in which a paper was cited by another when the user selects a node. The text containing all the citation context in the selected node is shown in another view.

Unlike the previous contributions, the following systems are not restricted to a particular document type, nor consider specific attributes. In this aspect they are similar to the system developed in this dissertation project, which does not rely on specific attributes. The FaceAtlas (CAO et al., 2010) visualization aims at showing the relationship among documents according to different facets of information. It consists of a graph visualization that shows the local relations and a density map to portray a global context. 
Figure 19 - The CiteSpace II system. A network of 541 co-cited authors. The thickness of each ring represent the amount of citations given a time slice and the color saturation the time of the corresponding citations. The prominent cluster (i.e. on the right) is the most recent one.

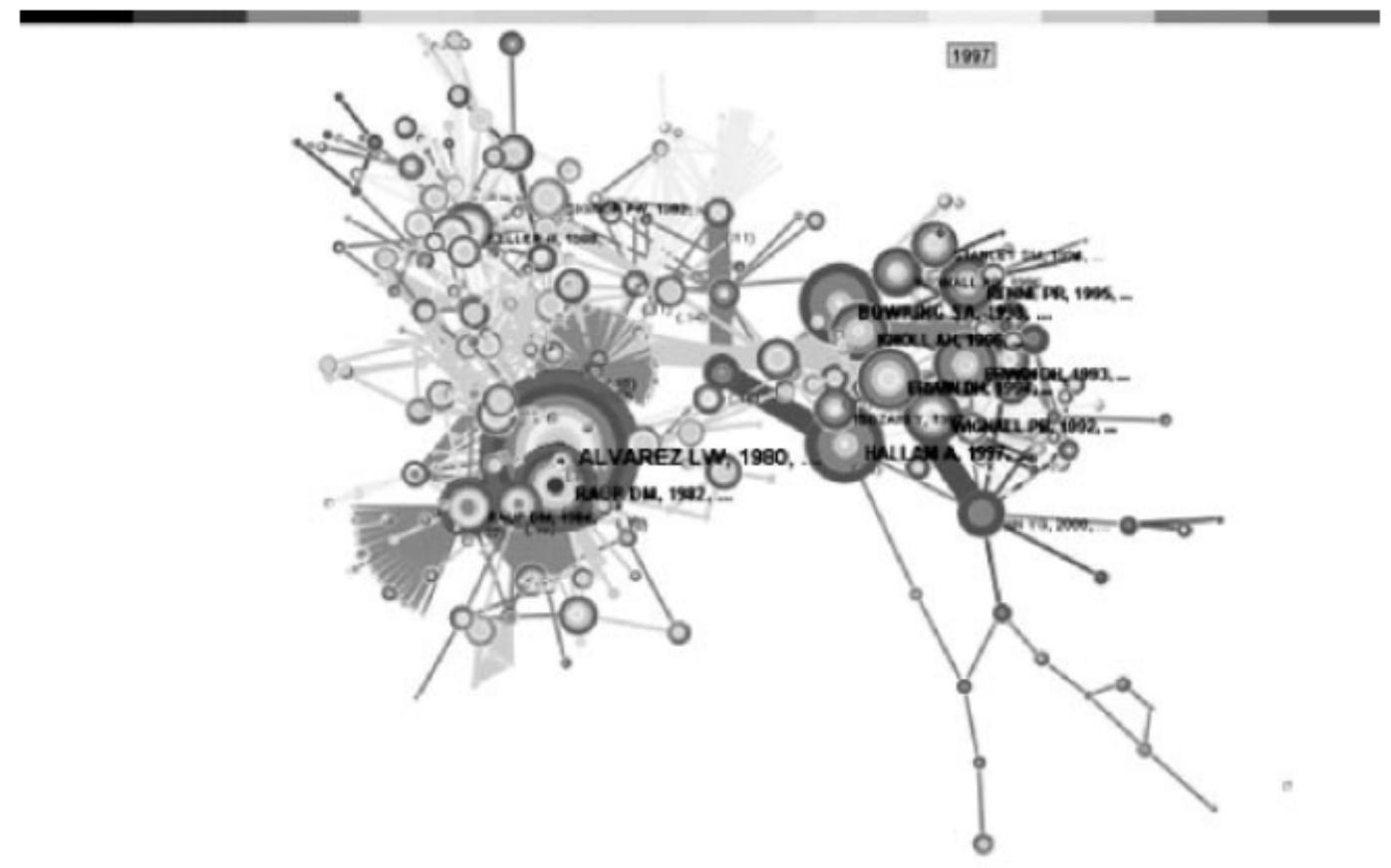

Source: Chen (2006)

Besides the common interactions such as zooming, filtering and highlighting, FaceAtlas also allows dynamic context switching for the user to change the primary visualization according to different facets. Showing relationships according to different facets is very interesting, since documents might be connected in different ways. To illustrate this idea, in Figure 21 it is possible to see the results for the query "diabetes". The color of the links and nodes represent the different facets where red represents symptoms, yellow diseases and green treatments. As the Figure shows, type-1 Diabetes and type-2 Diabetes (nodes in yellow) also share a lot of common symptoms (red link) but few common treatments (green link). It is possible to notice that diseases in the type-2 Diabetes's cluster share a lot of common treatments.

Another relevant system is Jigsaw (STASKO et al., 2007), which was developed in the context of investigative analysis and aims at helping analysts to explore reports collections in order to search, review and understand them better. The system connects entities such as people, places, dates and organizations from different reports, in order to help analysts to decide which reports they should read. The system offers four views: a tabular connections view to show the connection between the entities, a semantic graph view that shows the relation between the entities and the reports, a scatter plot view showing the relationship of any two entity categories and the text view that displays the original reports.

Even though Jigsaw was designed to help investigative analysts, it has been used in 
Figure 20 - The extended Revis visualization. A system to help users with the task of Systematic Literature Review. The red circles represent articles not included in the SLR whereas blue ones are included and gray ones are cited references.

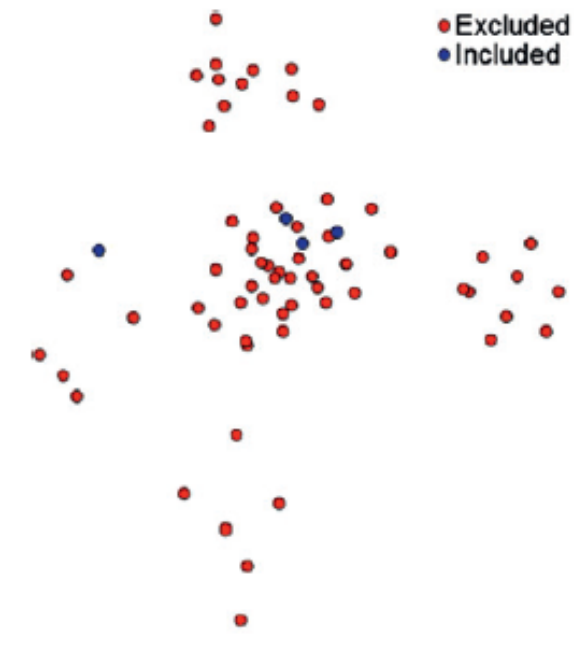

(a) Content map.

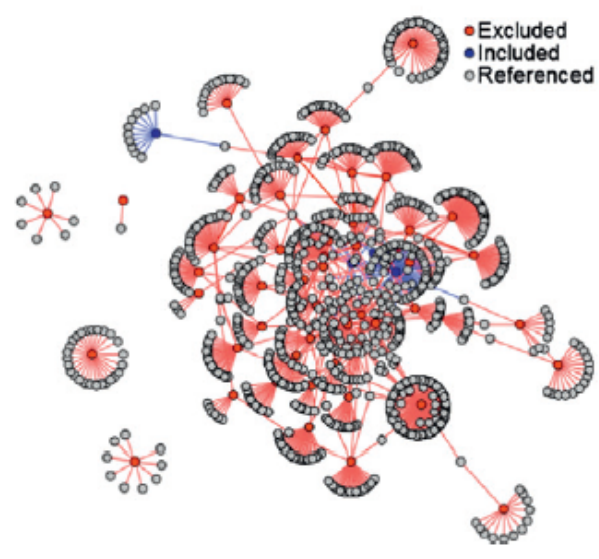

(b) Citation Map.

Source: Felizardo et al. (2012)

different domains because of its great generality on handling diverse types of documents. Improvements have been done in the system, as in (GöRG et al., 2013) which enhances it by including several automated algorithms such as analysis of similarities among document, document clusters, document sentiment and document summarization

DocuCompass (HEIMERL et al., 2016) presents a focus+context visualization by using a lens metaphor and the scatterplot visualization, where each circle represents a document. This study focuses on the exploration of a general text corpora and, by using lenses, the visualization helps users to analyze documents without loosing the overall context. An interesting feature in this visualization is the labeled bar charts (Figure 22). The circles in the scatterplot are clustered and mapped by color and each bar in a bar chart represents the frequency of a specific label in each group. For example, a user interested in the word 'texture' should explore the group colored in red. This can give users hints on where to search.

Users can define parameters such as the choices of dimensionality reduction technique and term selection method. A limitation is that it is not possible to open a document of interest. Another problem is that lenses allow users to focus in one area at a time, which makes it harder to compare groups of documents placed in different regions. Since the bar charts are positioned in a small space along with the term on the side, they are small to analyze and eventual cluttering happens with the text.

For simple queries that require one or few documents as result, the classic ranked lists provided by most search engines are usually sufficient. However, if a complex query is required or if the user doesn't have a clear idea on what s/he is looking for, new methods of exploration 
Figure 21 - The Face Atlas visualization. After the search for the keyword "diabetes", the system generated two clusters representing type- 1 and type- 2 diabetes. Red links represent similar complications whereas green links represent common symptoms.

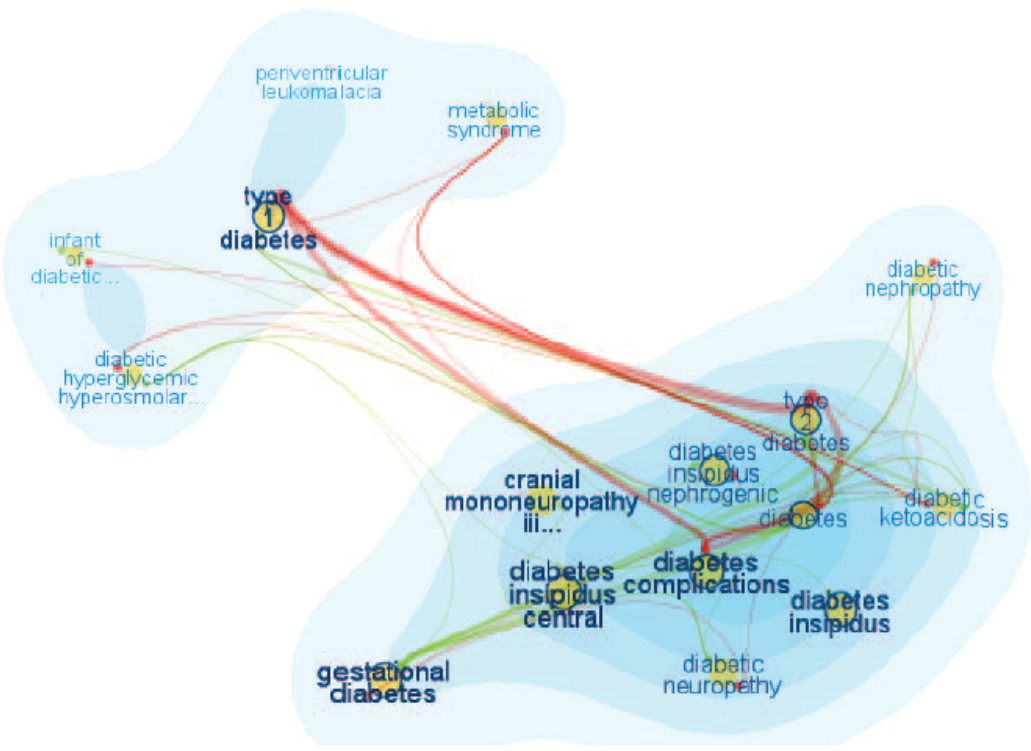

Source: Cao et al. (2010)

are necessary. Solutions that show relationship among documents by similarity are interesting in the sense that users are able to explore a document collection from different perspectives, which is important to acquire new insights and a clearer idea on how to formulate a good query. Studies comparing the CiteSpace system (CS) (CHEN, 2006) with the Web of Science (WoS) search engine, thus comparing an information visualization system to a textual based IR system have showed that users found the CS system more usable, easier to start searching and easier to search than the WoS. Moreover, the subjects of the experiment were more satisfied with the results when using the CS system than those using the WoS system.

\subsection{Understanding the search and the corpus}

Finally, the final category of contributions is considered more similar to the solution proposed in this dissertation, in that they provide three functionalities of interest to us, namely: to convey the relationship between the retrieved documents and the query and to convey the relationships among the documents, while focusing on improving document retrieval and supporting query refinement. Besides, we are also interested in handling the problem of vocabulary-mismatch by showing the different contexts a keyword of interest appears.

The solution by Tatemura (2000) (Figure 23) uses the concept of points of interest and allows a user to provide relevance feedback in order to refine an initial query. The Latent Semantic Indexing (LSI) technique is used to perform automatic indexing of documents, which uses multiple keywords to describe a document by implementing the VSM technique. This is 
Figure 22 - The DocuCompass system. The lens puts documents in focus and groups them by color. The bar charts represent the terms' frequencies in each group.

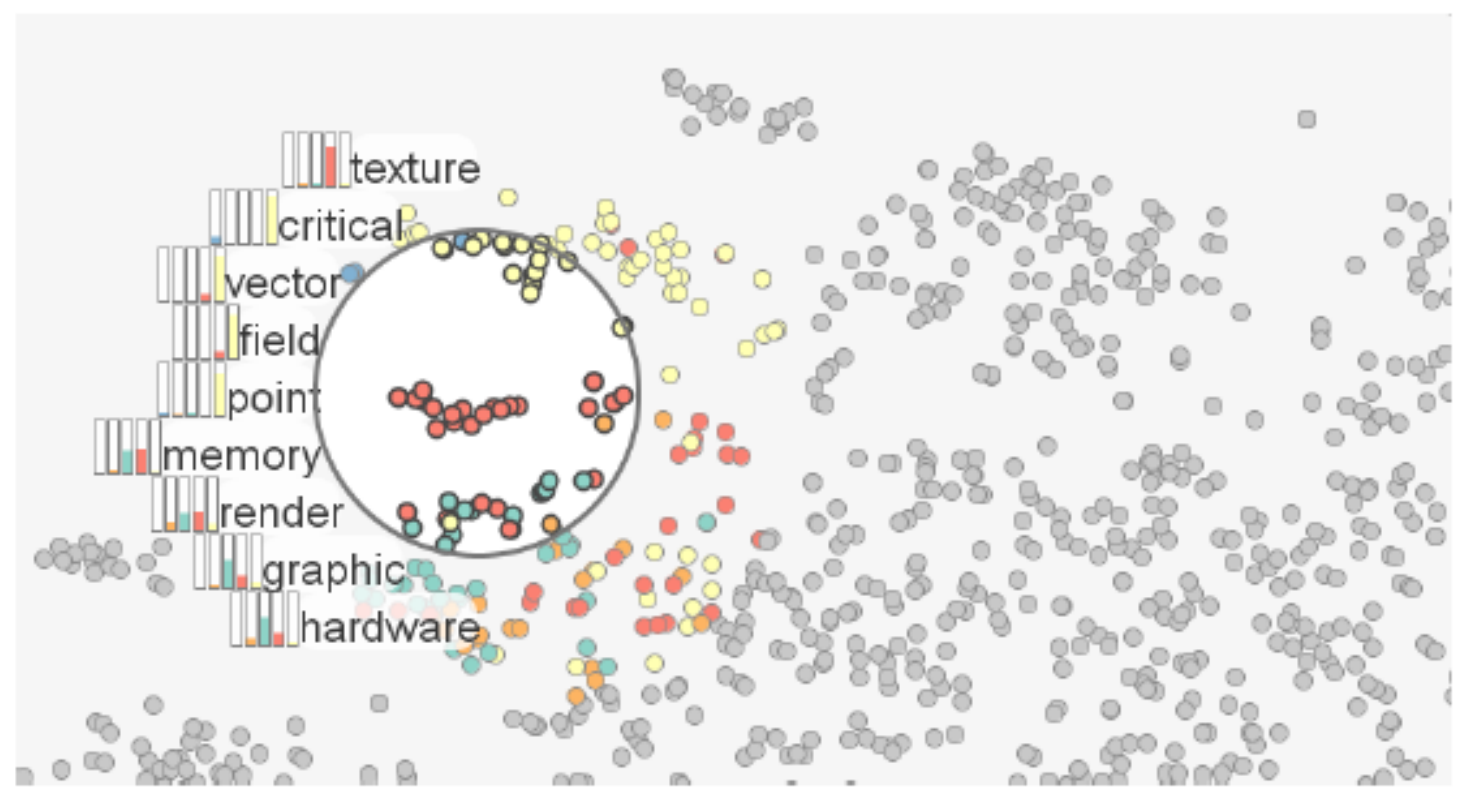

Source: Heimerl et al. (2016)

important because keywords might have multiple concepts and one concept can be described by multiple keywords. Documents and keywords are visualized in the 2D space and the user can choose to see documents and keywords separately.

Users can add multiple points of interest by choosing documents or keywords as anchors. By dragging a keyword or document, the objects' positions in the display are updated. It is also possible to select a document and read its full text. In order to see any relationship among documents and between documents and the query, the user must interact with individual documents, which could be difficult in a large text corpora.

The Citiviz tool (KAMPANYA et al., 2004) is a visual interface for the Computing and Information Technology Interactive Digital Educational Library (CITIDEL). The system shows the results of a keyword search into two main views. The first one is the hyperbolic tree which shows all documents relevant to the query into clusters according to how they fit into the ACM classification system. The user can click on a cluster to see the documents from that cluster in a 2D scatterplot. The circles in the scatterplot represents documents colored by the cluster they belong to. If a document belongs to multiple clusters, there would be a stack of circles colored according to the different clusters forming a tower. This allows the user to understand why a document was picked and its relation to the query. Moreover, if the user clicks on a tower, the system displays links connecting the document's citation.

The PEx-WEB system by Paulovich et al. (2008) (see Figure 24), uses the snippets from a web search engine or from a RSS source in order to build a graph-based visualization that shows how documents are related and possible patterns within the result. If documents are retrieved 
Figure 23 - The visualization with two anchors selected: the keyword "www" and the document "Finding and Visualizing Inter-Site Clan Graphs".

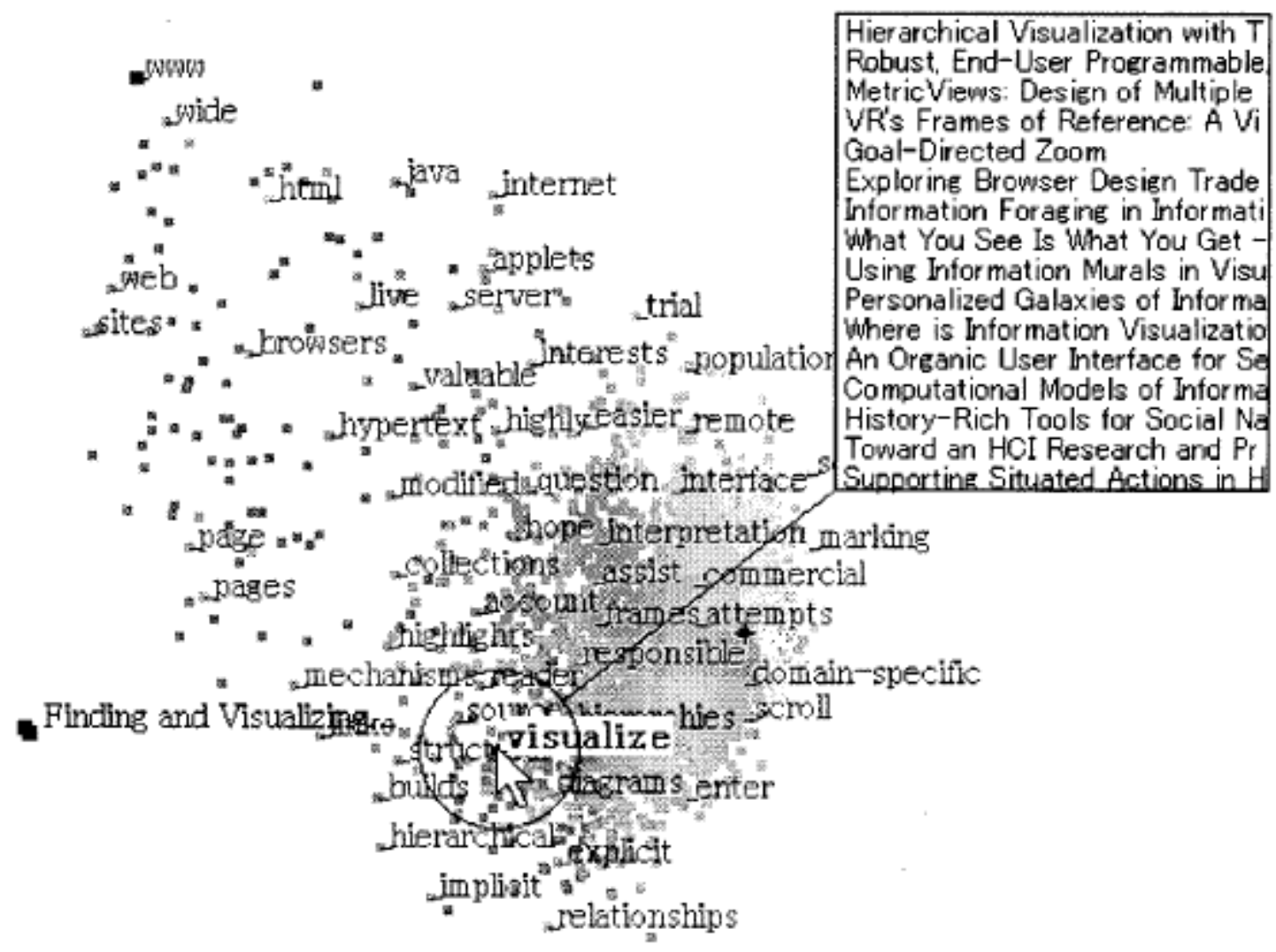

Source: Tatemura (2000)

with the Yahoo API, the visualization also shows the relationship between documents and the query by coloring or resizing nodes based on their ranking. The process is done in three main steps: obtain the VSM representation of the retrieved documents; calculate the distance among documents using the cosine-based distance and use the distance as input to the Least Square Projection (LSP) technique in order to retrieve the $\mathrm{X}$ and $\mathrm{Y}$ coordinates that the documents will be placed in the visualization.

Users can explore the results by clicking on a node to see a list of its nearest neighbors, selecting an area on the map in order to open the documents within that area and even train a classifier that uses the Support Vector Machine (SVM) model in order to color the circles in the visualization according to specific labels. Studies have shown that naive users could successfully use the system and it was possible to infer topics for a given region.

Similarly, the solution by Gomez-Nieto et al. (2014) aims to preserve the snippet structure provided by general search engines while showing relationship among the retrieved documents and the query. It uses the Least Square Projection (LSP) as the dimensionality reduction technique, since it preserves the neighborhood structure of documents and has low computational cost. Only 
Figure 24 - The Pex-Web visualization. The document are placed based on a similarity map and colors are assigned based on the frequency of the query on each document.

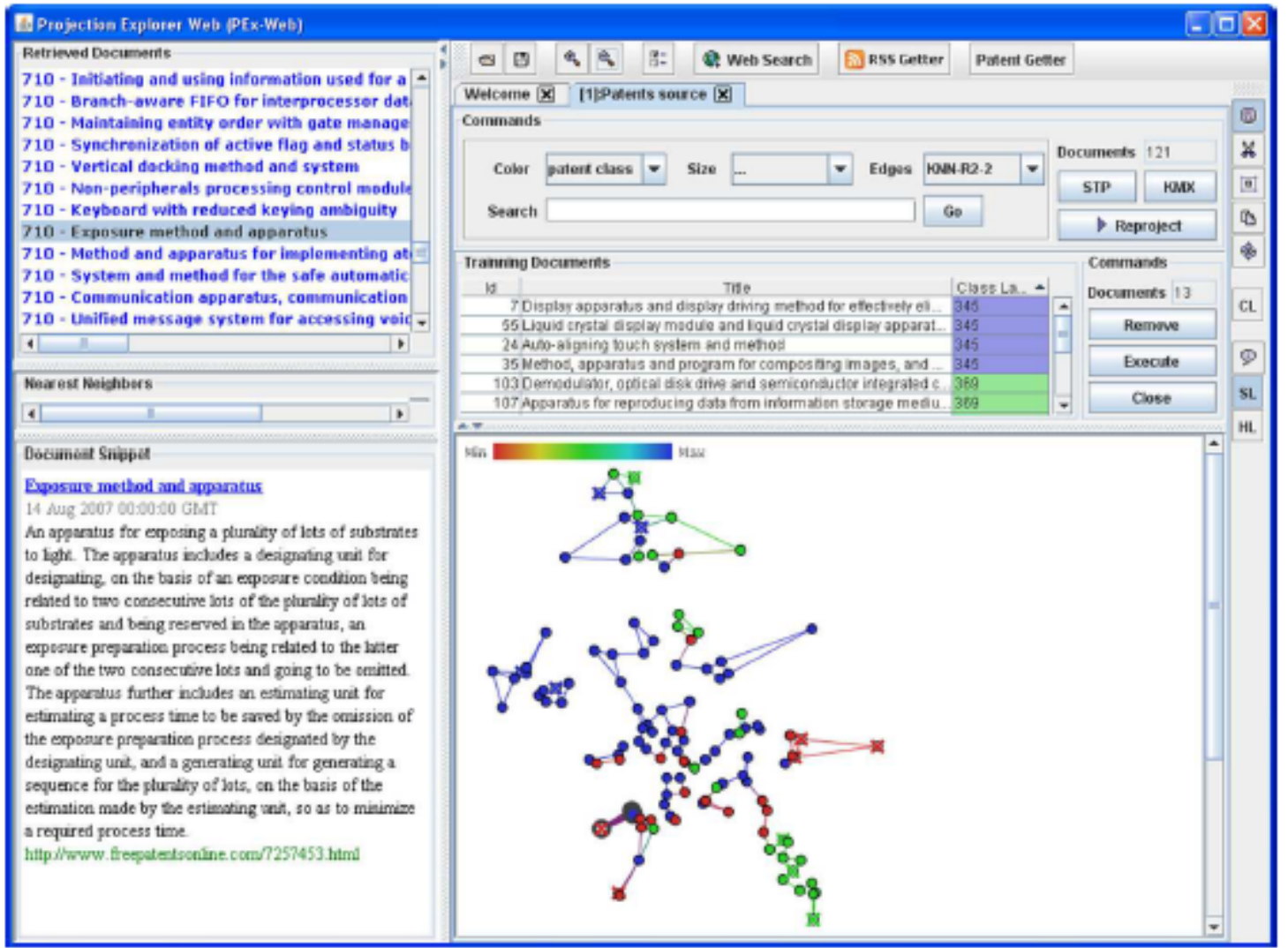

Source: Paulovich et al. (2008)

the summary of each document is considered in calculating the term frequency vector, which could result in cluster quality loss.

The two following systems differ from the previous ones since the user does not need to manually improve the query. The ForceSPIRE system (ENDERT; FIAUX; NORTH, 2012) was designed for high-resolution displays and its visualization pipeline is depicted in Figure 25 . The figure highlights the difference between a conventional visualization pipeline and the proposed one, where the soft data in the bottom is the resulting data after interpretation of the user's interactions. The work focuses on analyzing documents presented in a single view and positioned based on their similarity. The interactions within the visualization change the model because they update the importance value of entities (i.e., keywords), and the mass of each document (the number of entities associated to the document) thus, refining the original query. The system allows interactions such as highlighting (which adds importance to the text being highlighted), change document's position, search and annotation.

Bradel et al. (2014) enhanced ForceSPIRE and created the StarSPIRE system (Figure 26), also with the goal of shielding the user from the statistical model. This solution focuses on filtering out irrelevant data on a document retrieval process. The visualization consists of a graph 
Figure 25 - (top) The basic version of the "visualization pipeline". Interaction can be performed directly on the Algorithm (blue arrow) or the data (red arrow). (bottom) Modified version of the pipeline for semantic interaction, where the user interacts within the spatial metaphor (purple arrow).
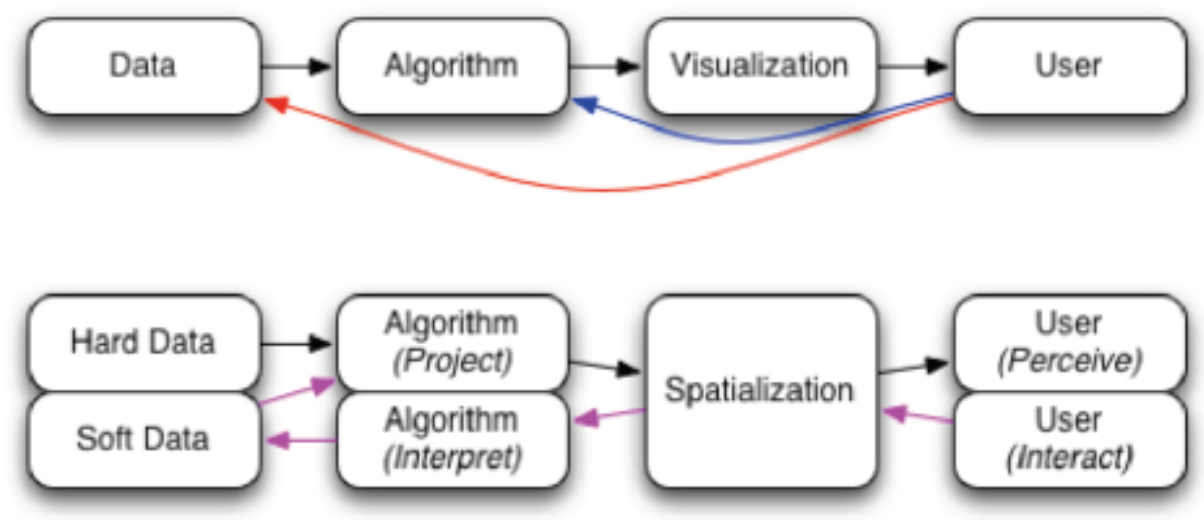

Source: Endert, Fiaux and North (2012)

using a modified force-directed layout where nodes are small boxes containing a summary of the document and their size and saturation represents its relevance to the query.

Figure 26 - StarSPIRE spatial workspace showing clusters of open documents and numerous iconified documents selected and arranged through semantic interaction.

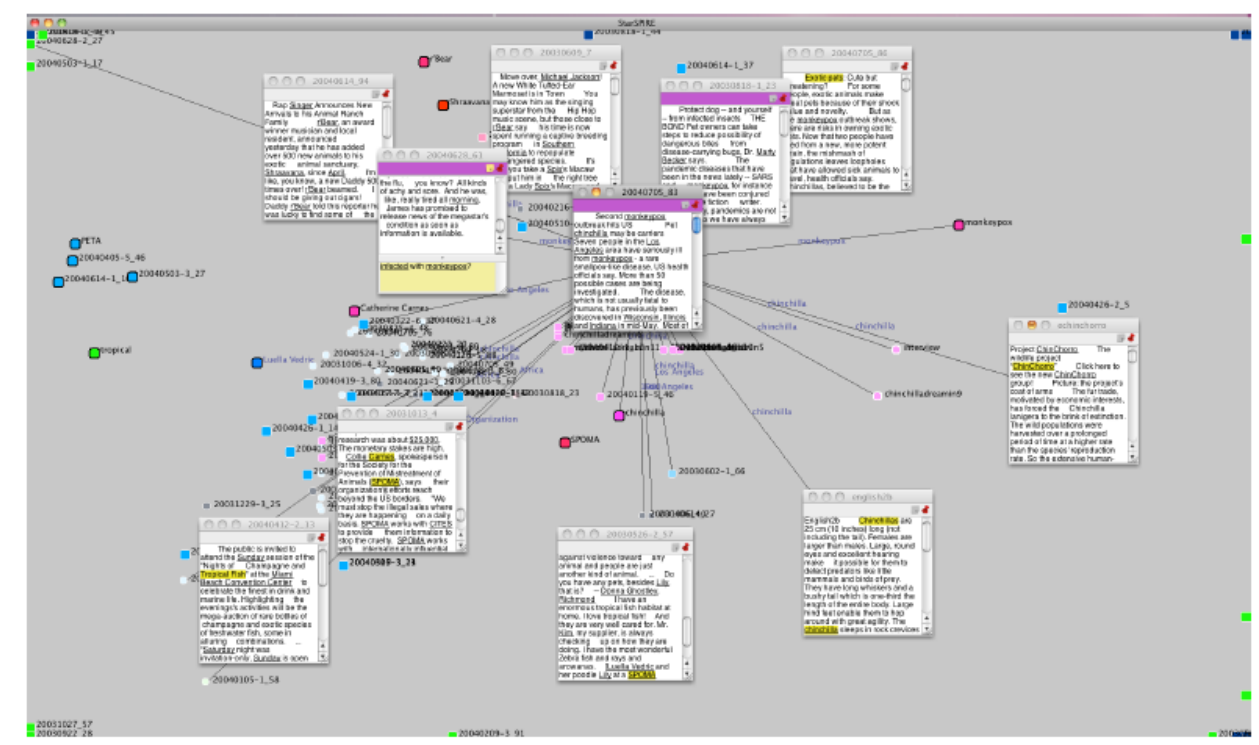

Source: Bradel et al. (2014)

The user can perform interactions such as searching, moving nodes, resizing a document, minimizing a document, annotations, searching, highlighting and overlapping documents. Besides, user can perform implicit or explicit queries where the former are resulted from the user interaction with the visualization and the latest the action of typing new keywords. By querying new documents, the system allows new information to be added to the display. Computational 
efficiency was improved, as compared to ForceSPIRE, because the system does not store most of the data and only displays a small set of documents. However, it is not recommended for an extremely large data set. Besides, user needs to interact with individual documents in order to add or remove relevance to it which can be overwhelming when dealing with a huge document corpus.

The contributions under this category tackle some of the problems we want to solve, but they do not consider the vocabulary-mismatch issue. It is also possible to note that graphs and scatterplot visualizations are the preferred techniques for solutions to show relationship among documents. The relationship to the query is usually conveyed by mapping the projection, such as increasing the size attribute of the nodes or circles according to the relevance of the document to the query.

\subsection{Discussion and final considerations}

The study by Liu et al. (2018) surveys interactive visualizations and text mining techniques aimed at supporting exploration and analysis of large-scale textual data. A visualization that links visualizations, tasks and mining techniques is available ${ }^{1}$.

The research showed that "typographic visualizations", "chart visualizations" , and "graph visualizations" are the most popular visualization techniques where the first includes text highlighting, word cloud and hybrid visualizations. The second consists of scatterplots, line charts, tables, and other charts such as bar charts. Finally, representatives of the third (graph visualizations) are node-link, tree and matrix visualizations. An interesting result was that even thought the largest number of papers retrieved was on "information retrieval", the survey identified 33 visualization papers and 1851 mining papers. Similarly, "classification" was addressed in few text visualization papers, even though it has been extensively studied in the text mining field. On the other hand, "exploratory analysis" and "natural language processing" were the largest and second largest clusters in terms of number of visualization papers.

By comparing the analysis tasks, some opportunities were identified in the study considering the gaps in text visualization and mining fields, such as creating solutions that solve "information retrieval" tasks with support from visualizations. For example, "query ambiguity", "federated search", and "distributed information retrieval" are challenges yet to be studied in the visualization field. Another opportunity is to include human knowledge to support text mining tasks. For example, "binary classification" and "recommendation" are well studied in text mining. However, the authors suggest that interactive visualizations could allow experts to provide informative supervision to the classifier and thus, improve the performance of text classification. Finally, another opportunity identified by Liu et al. (2018), related to our work,

1 http://visgroup.thss.tsinghua.edu.cn/textvis/ 
is that the majority of text mining techniques are not supported by existing studies on text visualization.

Table 1 shows how some of the solutions presented in this Chapter differ from each other. Three solutions from each category have been chosen based on the year of publication and perceived relevance. More specifically we analyzed the category of each system, if it uses metadata from a specific domain, if the solution uses some sort of text classification in order to enhance the visualization by suggesting new possible relevant documents and if the vocabulary-mismatch issue was handled. Additionally, Figure 27 shows the statistics of all the 30 solutions regarding the metadata usage, the text classification usage and if it tackled the vocabulary-mismatch issue.

Table 1 - Some of the solutions presented in the related work section regarding the A - Category (1: Understanding the search; 2: Understanding the corpus; and 3: Understanding the search and the corpus), $\mathrm{B}$ - if the proposed visualization requires metadata, $\mathrm{C}$ - if the solution counted on text classification in order to enhance the IR system and D - If the solution handled the vocabulary-mismatch issue.

\begin{tabular}{l|l|l|l|l} 
References & A & B & C & D \\
\hline Nguyen and Zhang (2006) & 1 & & & \\
Zimmer, Sahlgren and Kerren (2017) & 1 & & & \\
Choo et al. (2018) & 1 & & & \\
Rennison (1994) & 2 & & & \\
Cao et al. (2010) & 2 & & & \\
Heimerl et al. (2016) & 2 & & & \\
Paulovich et al. (2008) & 3 & & & \\
Bradel et al. (2014) & 3 & & & \\
Gomez-Nieto et al. (2014) & 3 & & &
\end{tabular}

Source: Developed by author.

Several solutions reviewed try to remedy the problem of retrieving relevant documents given a query where multiple documents might be relevant. However, many of them have been designed with a focus on specific types of documents, such as news articles or scientific papers. The contributions not targeted at a particular type of document do not support all the tasks relevant to this project, namely: to show relationship of documents to the query, show relationships among documents, allow query refinement, application of text classification algorithms and allow users to depict documents considering word semantics.

Even though the solutions presented in this chapter enlarge the state-of-art of visualizations that support information retrieval tasks, there is still space to evolve. Our goal is to create a visualization tool that helps the user to retrieve nearly all the relevant documents in a collection, thus supporting high recall tasks, in a more pleasant way than classic ranked lists. Moreover, we want to speed up the process by showing the similarities among documents and how the results relate to the query, providing recommendations of new documents using a text classification algorithm, allowing query refinement and handle the problem of vocabulary-mismatch. 
Figure 27 - Barchart showing the amount of Yes or No incidents for the following features: Does the solution use metadata information?, Does the solution deal with the issue of vocabulary mismatch? and Does the solution implement text classification algorithms in order to enhance the IR system?

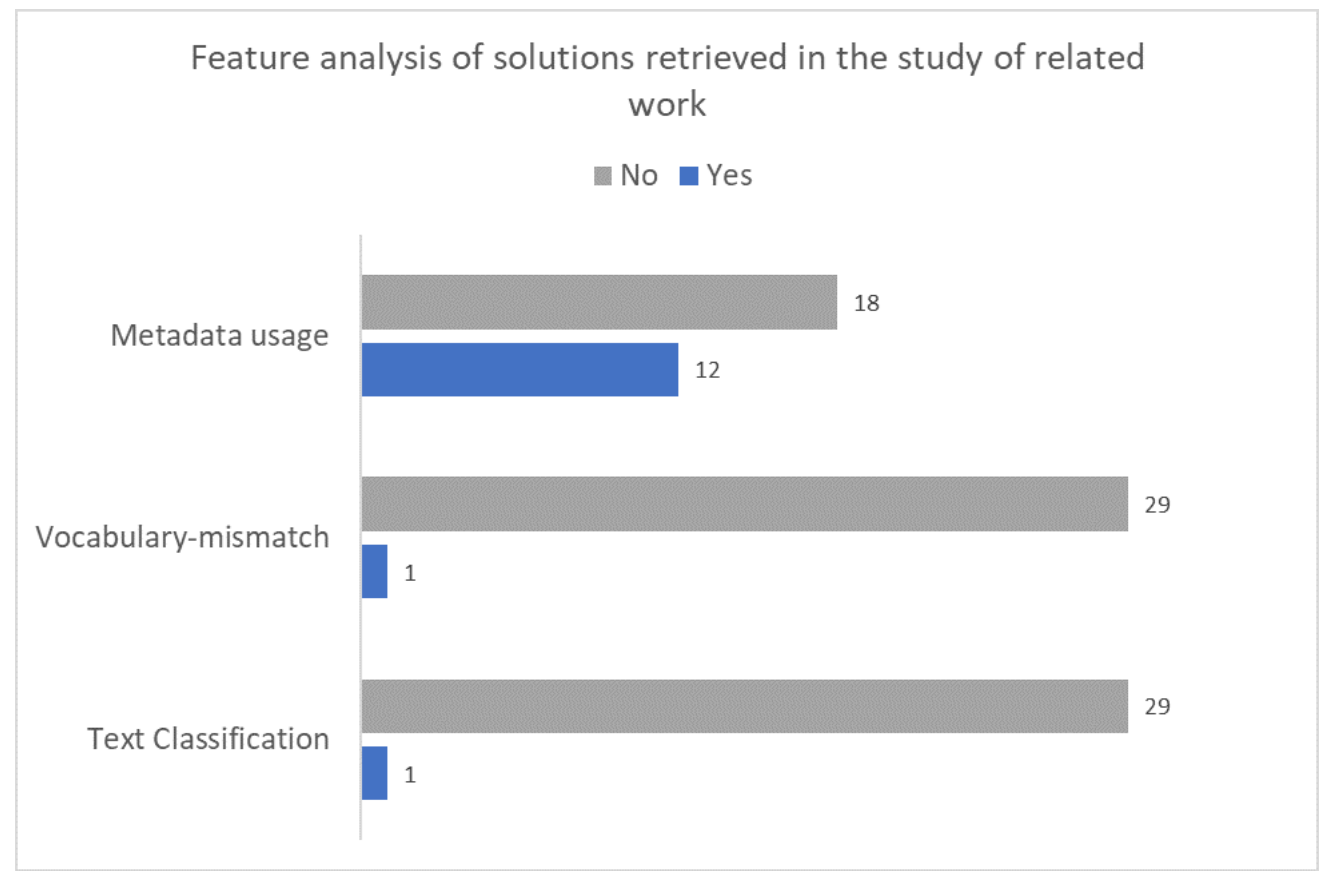

Source: Developed by author. 

CHAPTER

\section{4}

(1)

\section{TRIVIR: A VISUALIZATION SYSTEM TO SUPPORT DOCUMENT RETRIEVAL WITH HIGH RECALL}

\subsection{Initial Considerations}

In this Chapter we present TRIVIR, a visualization system to support document retrieval with high recall. We developed TRIVIR considering that current visualizations that support IR does not handle the vocabulary mismatch issue while integrating machine learning algorithms to suggest new documents. Besides, it provides ways for the user explore the collection by analysing how documents are related to each other and how the query is related among the result set. Additionally, we aim at connecting text mining techniques into a visualization system in scenarios where high recall is required.

This chapter is organized as follows: In section 4.2 we present an overview of the system, tasks supported, details on each TRIVIR's views, and underlying techniques used to develop the system. In section 4.3 we present our validation exercises using three different scenarios (a prelimiary validation, a literature review problem, and a systematic mapping study (SMS) scenario). Finally, section 4.4 concludes this Chapter with our final considerations.

\subsection{The TRIVIR System}

TRIVIR has been conceived as an interface to a general document retrieval system that implements a Continuous Active Learning (CAL) protocol. As such, it integrates machine learning and interactive text visualization for user-assisted document retrieval, in order to support high recall. Different alternatives could be considered as the underlying techniques, e.g., text representation, machine learning algorithm and multidimensional projection. Ideally, multiple 
choices could be offered for users to experiment and find out the best configuration for their particular retrieval task and document collection.

Users may choose the dimensionality reduction technique and the document representation since there is no technique that is better than all the others. Specially for the dimensionality reduction techniques, it is recommended that for each collection we would have a different approach that best represent its content (SEDLMAIR; MUNZNER; TORY, 2013).

The system interface is illustrated in Figure 28. It presents six views, namely: the Terms view (1), the Scatterplot view (2), the Document view (3), the Signature List view (4) which shares the same area as the Focus List and Suggestion List views. These three list views (Signature, Focus, and Suggestion) can be switched by clicking on the top buttons identified in (4) as "Signature", "Focus", and "Suggestion". The Signature List view is the default at system start-up, and the two other views are illustrated side by side in Figure 29.

Figure 28 - TRIVIR interface. The Scatterplot View in area (2) shows a similarity map depicting a collection of 675 papers in Computer Science. The circle colors indicate the current query document (green), and then: the relevant (blue), not relevant (red), suggested as relevant (yellow), and yet unlabeled documents (gray). The menu on top allows filtering the scatterplot in three different ways ( $2 a, 2 b$ and $2 c$ ). The Terms View (1) shows the important terms from the query document, where the user can remove (1a) or add (1b) terms. Area (4) shows the Signature List view, which shows the relevant 3-grams in the corpus. It can be switched with the Focus List or the Suggestion List views by clicking on the corresponding buttons. The user can visualize important 3-grams from the collection, select 3-grams with specific terms (4a), or select all 3-grams that include terms from the terms view (4b).

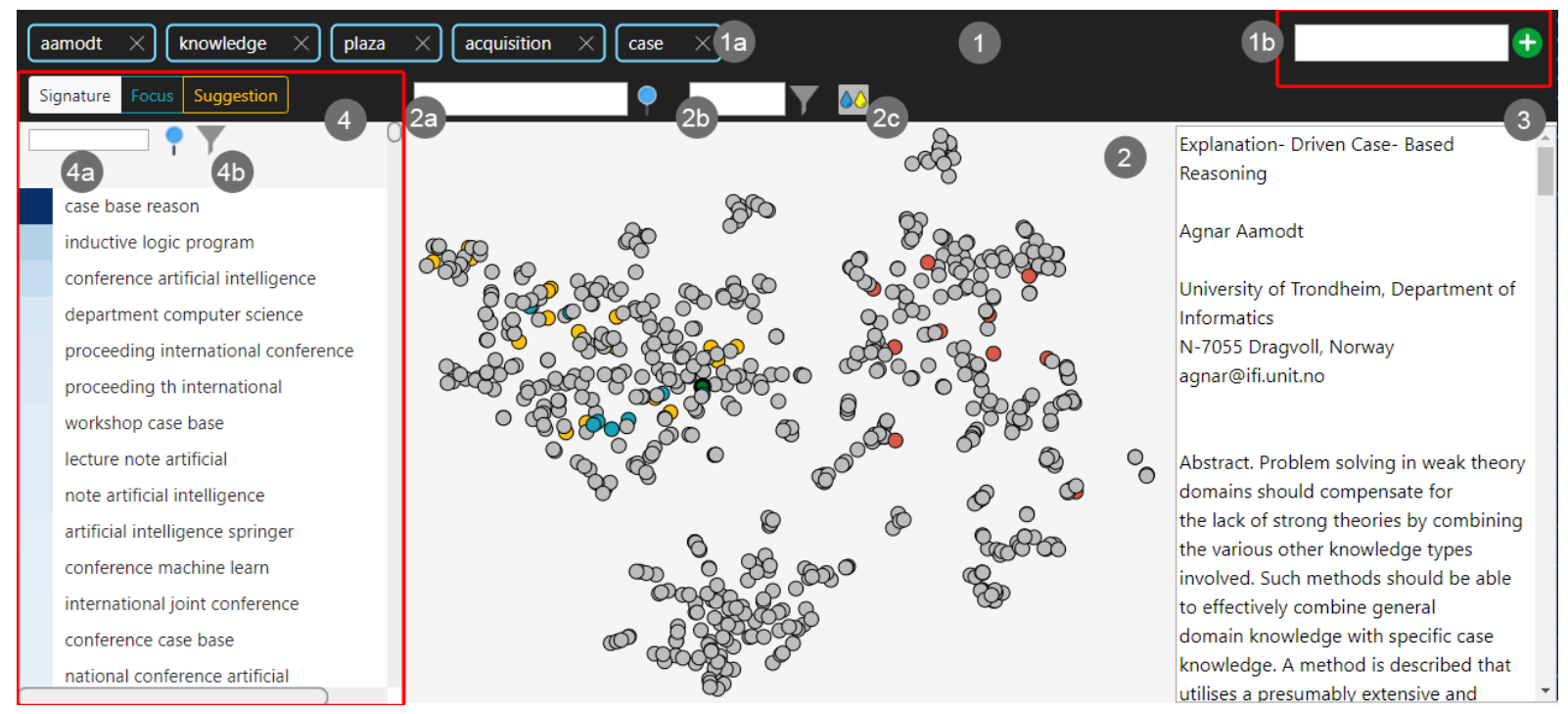

Source:Developed by author

In this example, we use a collection of 675 papers from four different Computer Science areas (Case-Based Reasoning (CBR), Inductive Logic Programming (ILP), Information Retrieval $(I R)$ and Sonification (SON)) containing title, authors, affiliation, abstract and references. The circle colors in the Scatterplot view indicate the current query document (green), relevant 
documents (blue), not relevant documents (red), suggested documents (yellow), and not yet labeled documents (gray). A user can select documents including a particular term (2a); filter the $\mathrm{K}$ documents most similar to the initial query (2b); and reduce point clutter by displaying only the relevant and suggested documents (2c). Clicking on a circle displays its content in the Document View (3) (query document shown). The terms view (1) shows the important terms from the query document, where the user can remove (1a) or add (1b) terms as needed. The Signature List view shows the relevant 3-grams in the corpus. The user can select 3-grams with specific terms (4a), or select all 3-grams including terms from the terms view (4b). The focus list would display the titles of all documents currently labeled as relevant, the suggestion list would display the title of the documents labeled as relevant by the ML algorithm as well as a button to retrain the classifier.

Figure 29 - Both the Focus List view (1) and the Suggestion List view (2) share the same area in the interface, and the user switches between them (and the Signature List view) by selecting the corresponding buttons on the top (refer to Figure 28). The Focus List view shows all documents currently labeled as relevant; the Suggestion List view shows the documents suggested as relevant by the ML classifier. The user can press the "Train" button to retrain the classifier (2a).

\begin{tabular}{|c|c|c|c|c|c|}
\hline Signature & Focus & Suggestion & Signature & Focus & Suggestion \\
\hline \multicolumn{6}{|l|}{ total: 11} \\
\hline \multicolumn{3}{|c|}{ Explanation- Driven Case- Based Reasoning } & \multicolumn{3}{|c|}{ Reasoning with Reasons in Case-Based Comp } \\
\hline \multicolumn{3}{|c|}{ Explanation-based Similarity: A Unifying Appro } & \multicolumn{3}{|c|}{ Separating the Cases from the Data: Towards } \\
\hline \multicolumn{3}{|c|}{ Representing Knowledge for Case-Based Reasc } & \multicolumn{3}{|c|}{ On the Automatic Generation of Case Librarie } \\
\hline \multicolumn{3}{|c|}{ A Hybrid Knowledge-Based System for Technic, } & \multicolumn{3}{|c|}{ Learning to Refine Indexing by Introspective } \\
\hline \multicolumn{3}{|c|}{ Systems, Tasks and Adaptation Knowledge: Rev } & \multicolumn{3}{|c|}{ Route Planning by Analogy } \\
\hline \multicolumn{3}{|c|}{ Integrating Rules and Cases for the Classificatic } & \multicolumn{3}{|c|}{ Case Adaptation Using an Incomplete Causal } \\
\hline
\end{tabular}

Developed by author

The system allows the user to explore a document collection and label documents as relevant or not relevant through exploration of the similarity map among the result set and the query, through classic ranked lists and through exploration of how key terms are used in the text.

\subsubsection{Tasks supported}

A user should be able to explore the collection, read individual documents (Document view) and label documents as relevant or not relevant, assisted by the information displayed in the different views. It is possible to label one document at a time through classic ranked lists (Focus List and Suggestion List views) or through the Scatterplot view. The user might also 
want to label multiple documents simultaneously based on how key terms are used within the documents (Signature List view) and by letting the system automatically label all documents similar to a selected one as relevant or not relevant (Scatterplot view). Additionally, the user can retrieve synonyms and terms used in the same context as a selected one, thus expanding the set of important terms (Terms view). As users find out actual word synonyms and alternative words that occur in the same context as the selected, they may be able to identify additional relevant documents, e.g., that use different words to describe the same concept, thus handling the vocabulary mismatch issue.

An overview of the system's flow is presented in Figure 30. The user first selects a corpus and a query document. At this point, s/he has the option of changing the document representation, dissimilarity function and/or the multidimensional projection technique. As default, TRIVIR uses as document representation a Vector Space Model (bag-of-words) with a TF-IDF weighting scheme, the cosine similarity and the t-SNE projection technique to create the $2 \mathrm{D}$ similarity scatterplot of the entire collection. The system first computes and stores both the word embedding and the VSM document representations as well as the model for the collection using the fastText library for R (JOULIN et al., 2017) which consists in using the skipgram model to create word representations of the collection (further explained in Sub-section 4.2.3). The model is used to generate the synonyms based on the context of the terms and applied to the Terms view. Then, the 3-gram list is created and ranked by decreased frequency. Finally, given the selected document representation, it computes the pairwise cosine similarities from the query document to all the others. The system can then load the Terms View, Signature List view, Focus List view and Document view. The ML algorithm (currently, the system uses the classifier of the fastText library (BOJANOWSKI et al., 2017)) is trained considering the 10 most similar documents to the query document as relevant, and the 10 less similar as not relevant. The algorithm returns 20 possibly relevant documents, which are included in the Suggestion List view. Finally, the Scatterplot view is loaded based on the documents' labels.

The user can interact with the Scatterplot, Suggestion List, Focus List and Signature List views to label new documents. Along with the other views, the Terms view and the Document view support the exploration of the collection. The user can retrain the ML algorithm as much as s/he wants and stop the process at any time. At the end, the Focus List view with the relevant documents is saved. More details on each view is given next.

\subsubsection{The views detailed}

The Terms view shows the five most important terms from the query document according to the TF-IDF weighting scheme. For each term a list of synonyms is retrieved using two different $\mathrm{R}$ libraries. The first one is $q d a p^{1}$, which has a functionality to retrieve the synonyms from an

$\overline{1}$ https://CRAN.R-project.org/package=qdap 
Figure 30 - Information flow in TRIVIR. The user first selects a corpus and a query document, and possibly modifies the default settings. The system initially renders the Document view and the associated Terms, Signature List and Focus List views, which are computed considering the document representation, the creation of a model containing the word representation of the collection using the skipgram model, the ranked 3-gram list, and the distance function. The ML algorithm is trained; the documents it predicts as relevant define the Suggestion List view. Finally the 2D Scatterplot view is created and rendered, with the circles colored according to the documents' labels. Interacting with the multiple views the user has the necessary support to investigate the collection and decide about document relevance. The CAL protocol allows her to retrain the ML classifier and finish the process at any time, when the final Focus List view gives all the documents identified as relevant.

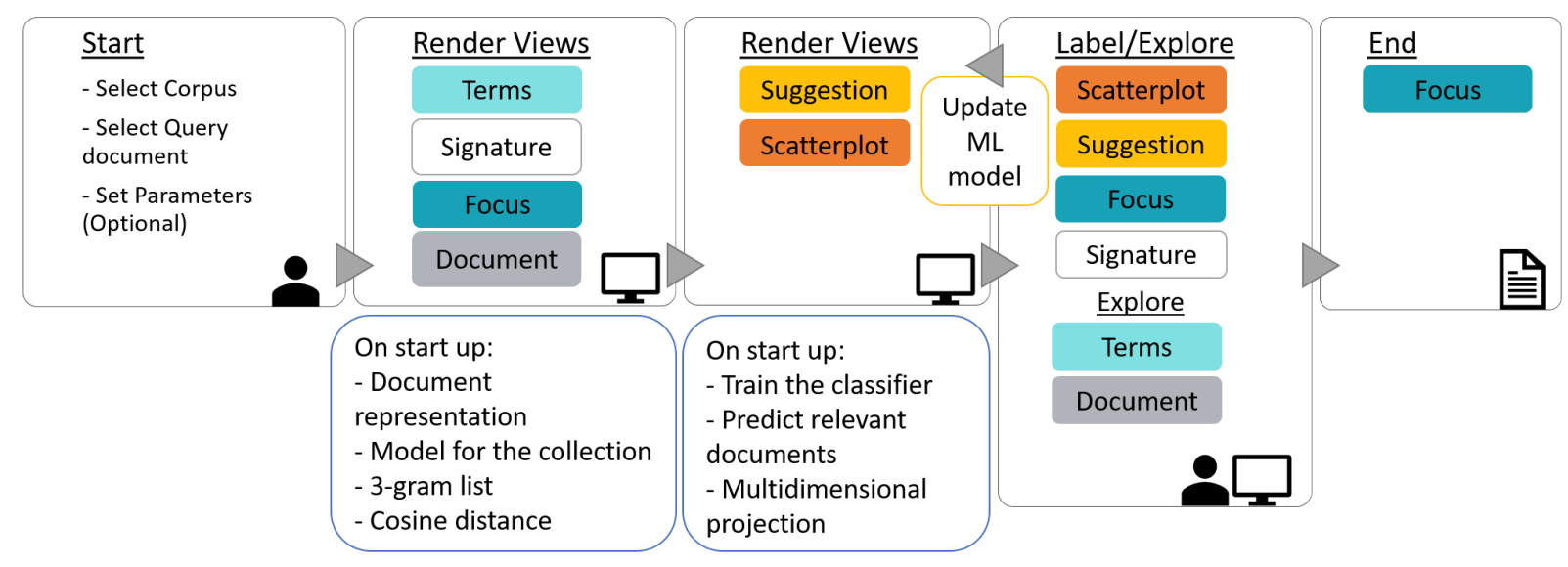

Developed by author

online dictionary (RINKER, 2019), and the second one (fastrtext ${ }^{2}$ ) has a method to retrieve the words used in the same context as a specific term (BOJANOWSKI et al., 2017). The results are merged and presented as a list when the user clicks on a term. Figure 31 presents a list of synonyms for the term "case" and, since one of the groups is of papers in the area of Case Base Reasoning, the terms "reason" and "base" usually appear in the same context as the word "case" and thus, appear in the list. The user can also add (Figure 28 (1b)) and remove (Figure 28 (1a)) terms as needed. However, adding or deleting new terms does not affect the model used by the classifier nor the visualizations. In fact, the manipulation of the term's view only occurs in connection with the Signature List view.

The Signature view was inspired by the solution by Jankowska, Keselj and Milios (2012), who used character n-grams for text classification based on the Common N-Gram (CNG) analysis method. They presented the Relative N-Gram Signatures visualization that allows the user to explore the interesting n-grams for a document or group of documents and to get insight into the inner workings of the CNG classifier. Our Signature List view is simpler than theirs, however, it helps the user to gain insight on common or interesting n-grams in the corpus. It consists of the n-grams (so far we adopt $n=3$ ) of the entire collection ordered by decreasing frequency. The 
Figure 31 - List of synonyms for the term "case". The list merges dictionary synonyms and terms that are used in the same context as the selected one in the collection.

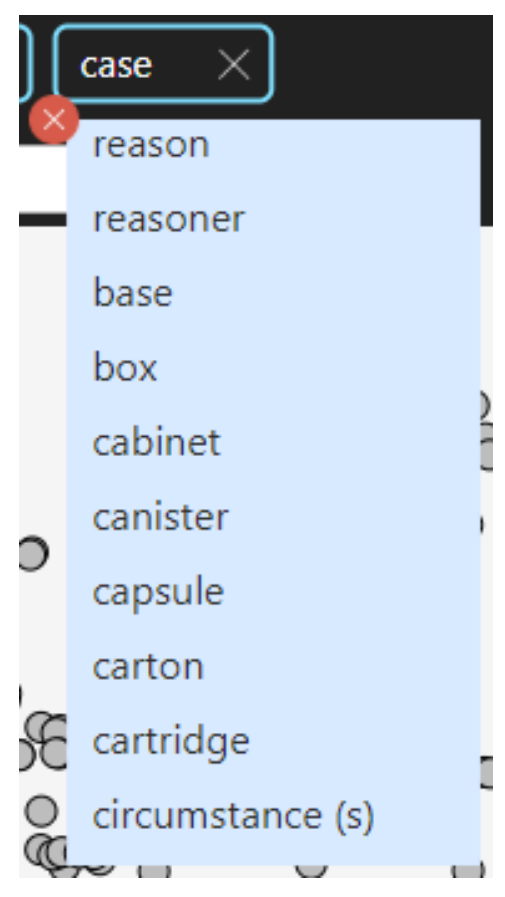

Developed by author

3-grams with frequency equal to 1 were removed, since keeping them significantly increased the list size. The blue bars on the left represent the frequency of the 3-gram where the highest is the saturation, the highest is the frequency. The goal in providing the Signature List view is to allow users to identify a term's context of usage and filter the Scatterplot view to to display only the documents with a specific 3-gram (the other documents are hidden until the user resets the Scatterplot view). The user can either type in terms in the Signature List view (Figure 28 (4a)) or filter the 3-grams that contain the terms in the Terms view (Figure 28 (4b)). In the last case, the terms in the synonyms lists are not considered since they are only suggestions to the user. Further, s/he can label as relevant all documents that contain a particular 3-gram (Figure 32 (a)) or likewise as not relevant (Figure 32 (b)). However, documents already labeled as relevant, not relevant or the query document are not affected. This functionality allows users to manually select the context of a term s/he is or is not interested. Both the Terms view and the Signature List view tackle the problem of vocabulary mismatch where the former deals with different words describing the same concept and the latest deals with the same word being used with different meanings, or in distinct contexts.

The Scatterplot view represents the entire collection, where each document is depicted as a circle, at the spatial location computed by a multidimensional projection technique. Our interest is on projection techniques, as described in Chapter 2, aimed at preserving the neighbourhood relationships between the data elements, in this case, the individual documents. Thus, it is expected that similar documents are placed close in the projection. In the Scatterplot view, 
Figure 32 - Using the Signature List view to filter the scatterplot to display the documents that include the 3-gram "case base reason". One observes four categories of documents in the Scatterplot view (all of them include this 3-gram): the query document (green), relevant documents (blue), suggested documents (yellow) and not yet labeled documents (gray). The user can set all not labeled documents as not relevant (a) or relevant (b) at once.

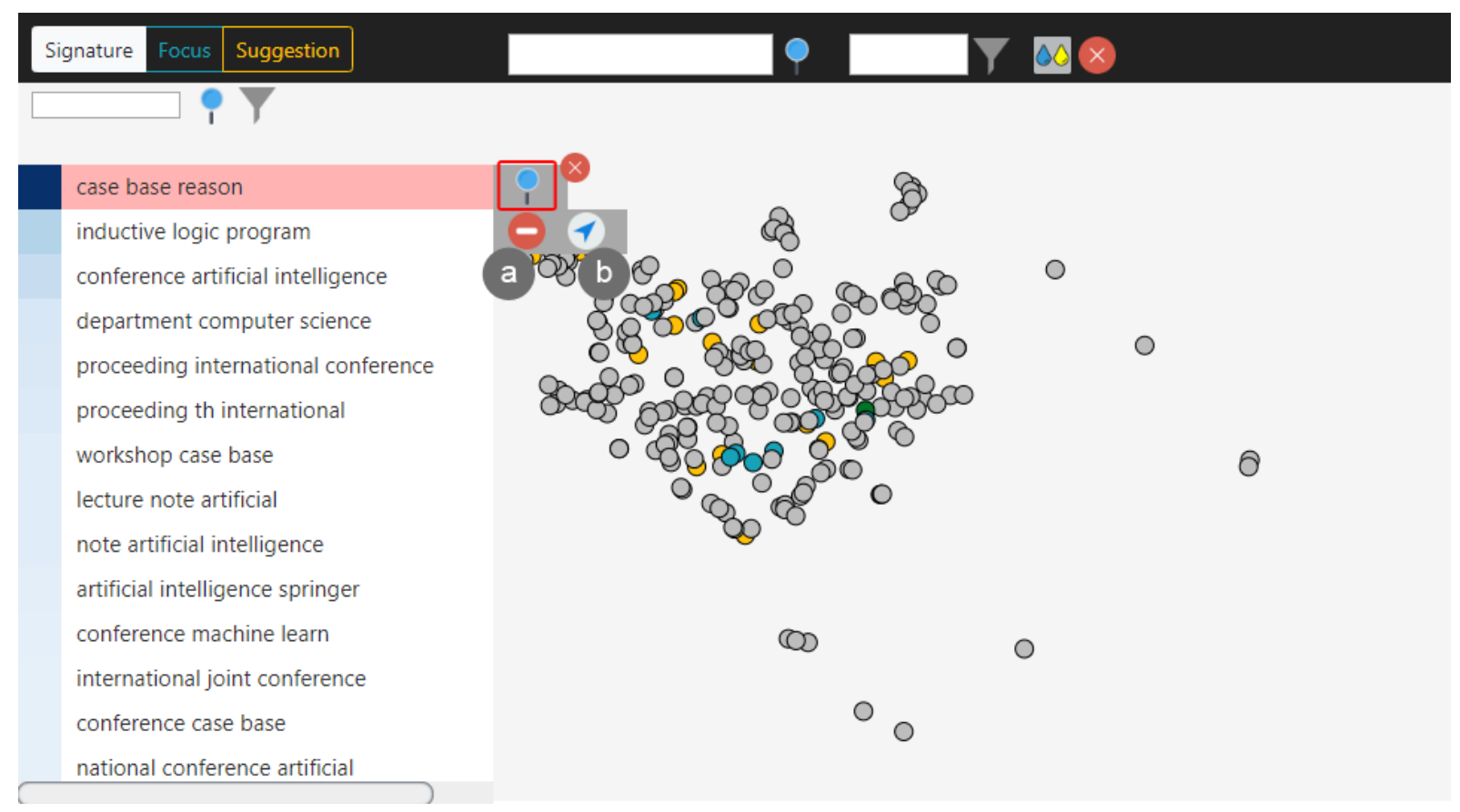

distinct colors indicate, respectively, the query document(s) (in green), the documents labeled as relevant (in blue), the documents labeled as not relevant (in red), the suggested documents (in yellow), and documents not yet labeled (in gray). Clicking on a circle displays its full text in the Document view and pops a menu right above it, which can be used to label the document as relevant/not relevant. The menu that is shown is different depending on the label of the selected document (Figure 33). For the suggested and not labeled documents, the user can use the menu to set a selected document as relevant or not relevant. However, for the relevant documents, the user can set a specific document as an additional query document, in which case the circle will be colored green, and its 10 most similar documents are also set as relevant (Figure 34 (a,b)). Similarly, if the same option is chosen for a not relevant document, its 10 most similar documents are set as not relevant (Figure $34(\mathrm{c}, \mathrm{d})$ ). The classifier will not be retrained at this point, unless the user clicks on the "Train" button in the Suggestion List view. The Scatterplot view also offers some searching functionalities, such as finding documents with a particular term or phrase (Figure $28(2 \mathrm{a})$ ), or filtering the map to display only the $k$ most similar documents to the initial query document (Figure $28(2 \mathrm{~b})$ ), or to show only the relevant and suggested documents (Figure 28 (2c)). This is particularly helpful when working with large collections, as the similarity map can become highly cluttered.

As mentioned, TRIVIR relies on a ML algorithm to recommend new documents. The list of recommended documents is shown in the Suggestion List view (Figure 29 (2)). The user 
Figure 33 - The menu on the Scatterplot view has different options depending on the label of the document. For not labeled (gray) and suggested documents (yellow) the user has the option of setting a document as relevant or not relevant (a). For relevant documents (blue) the user can set it as not relevant or query (b) and for not relevant documents (red) the user can add them to the Focus list (set as relevant) or set other documents similar to it as not relevant (c)

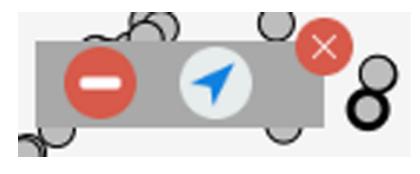

(a) labeled and suggested

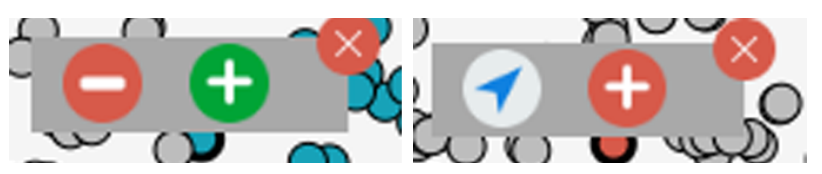

(b) relevant

(c) not relevant

Developed by author.

can click on a document from the list, and a menu is displayed that allows labeling the document (Figure 35). S/he can also read the selected document in the Document view and locate it in the Scatterplot view (the circle that represents the selected document is shown with a thicker border). A document set as relevant will be transferred to the Focus List view (Figure 29 (1)) and its circle on the Scatterplot view will be colored blue. If the user sets a document as not relevant its circle is colored red in the Scatterplot view. The user can choose to retrain the ML algorithm at any time by clicking on the "Train" button (Figure 29 (2a)) - upon which any remaining documents in the list are considered not relevant, i.e., the user is not required to label every document in the Suggestion List view. The classifier uses all documents in the Focus List and the documents labeled as not relevant to train the algorithm and suggest new documents.

Finally, the Focus List view (Figure 29 (1)) includes all documents currently deemed as relevant. It is possible to click on an item in the list to load its content in the Document view, and remove a specific document from it (Figure 36). In the end, it is expected that the Focus List view will include all the relevant documents for a given query.

\subsubsection{Underlying techniques}

Currently, two alternative approaches are supported for representing text documents: Word Embeddings using fastText (BOJANOWSKI et al., 2017), or the VSM using the TFIDF term weighting (JONES, 1988). The corpus is pre processed to remove stop-words and non letter characters. Moreover, all characters are lower cased and lemmatization is applied, which consists in transforming a word into its root or dictionary form. The system supports two multidimensional projection techniques to create the 2D similarity scatterplot: (1) The Barnes-Hut implementation of the t-SNE algorithm (MAATEN, 2014a), and (2) the LeastSquare Projection (LSP) (PAULOVICH et al., 2008). In case the LSP projection is chosen, the pairwise cosine similarity measure is applied on the documents' vector representation of the entire collection, query included.

The CAL protocol has been adopted based on previous evidence that it yields superior 
Figure 34 - Demonstration of how to expand the set of relevant (by creating additional query documents) and not relevant documents. The example illustrates the before and after setting a document as query ( $a$ and $b$ ) and the before and after expanding the set of not relevant documents ( $c$ and d)

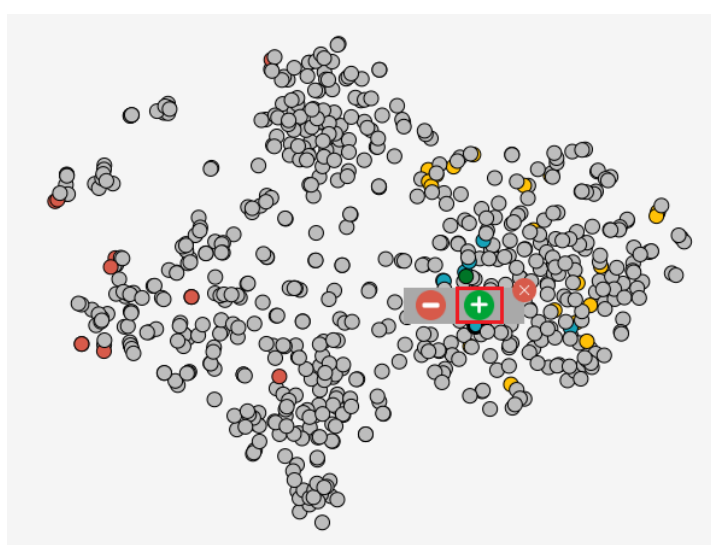

(a) Before setting a document as query.

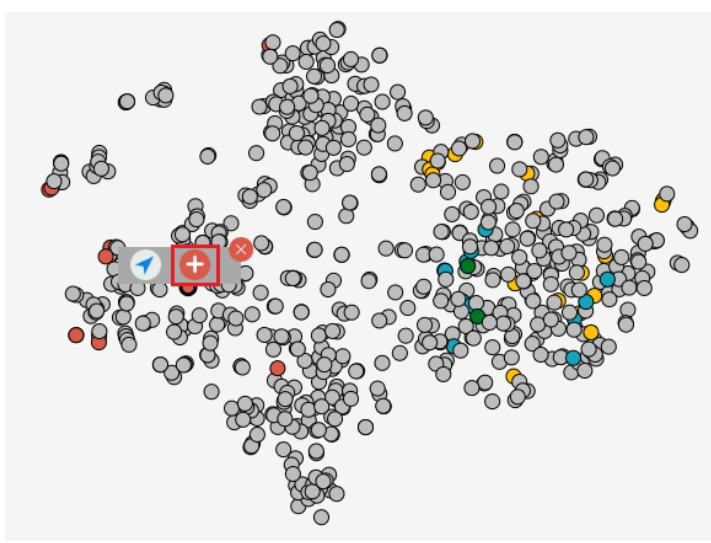

(c) Before increasing the set of not relevant.

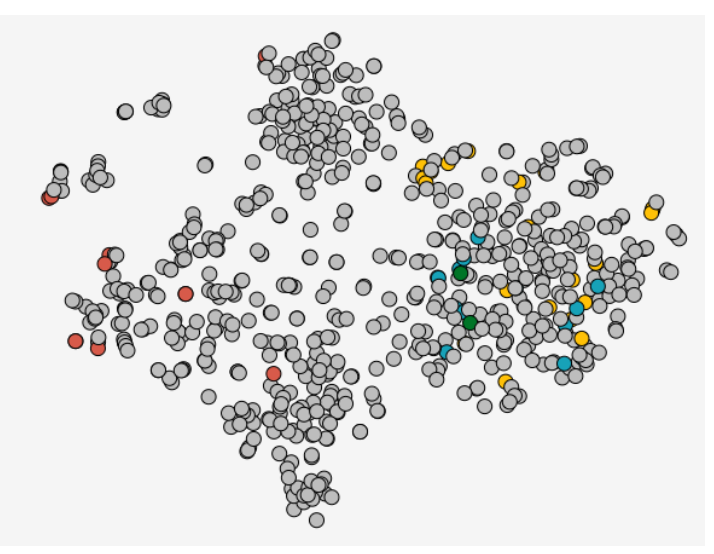

(b) After setting a document as query.

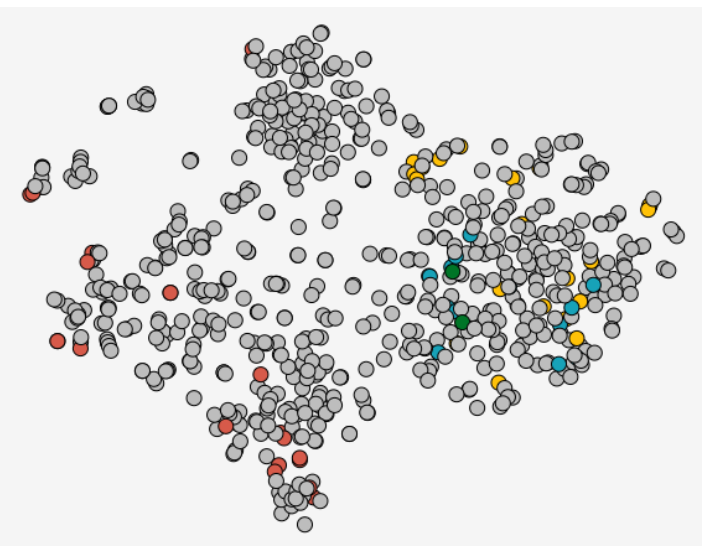

(d) After increasing the set of not relevant.

Developed by author.

Figure 35 - Suggestion View menu. The user has the options to set a document as not relevant or relevant.

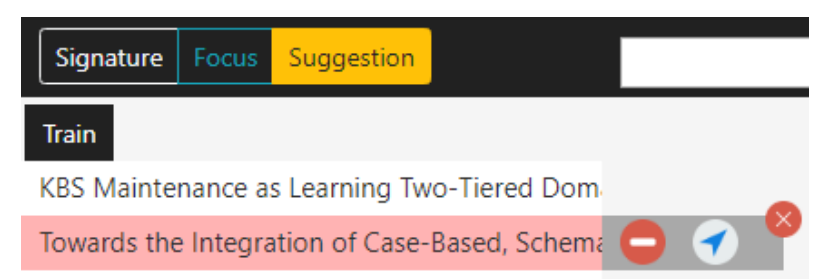

Developed by author

results while demanding less review effort (GROSSMAN; CORMACK, 2016). The choice of TAR protocol determines the choice of the underlying classification algorithm. Currently, the system uses the classifier from the fastText library (JOULIN et al., 2017), using multiple relevance rather than binary relevance. We found this necessary after including a functionality that allows the user to indicate additional query documents. By automatically setting as relevant 
Figure 36 - Focus List menu. The user has the option to remove the document from the list.

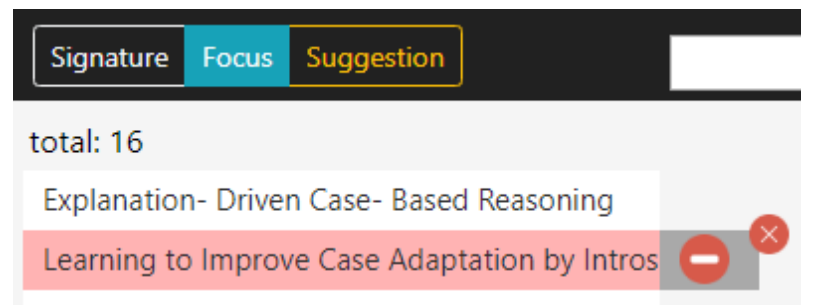

Developed by author

the 10 most similar documents to the new query, we observed a decrease in precision, as not relevant documents were added to the Focus List view, with negative impact on the classifier performance. Thus, we established four possible classes for each document: all documents considered not relevant are in the same class (1) Not Relevant, whether the ones considered relevant are split into classes (2) Relevant Query, (3) Relevant, (4) Relevant Automatic, and assigned different weights (2, 1 and 0.5 , respectively). Since the Suggestion List view only ranks 20 documents at a time, the documents are ordered based on their weights, which results in showing first the documents in the same class as the query documents, followed by the ones labeled as relevant and finally, the ones set automatically as relevant.

TRIVIR performs the following steps using fastText functions of the fastrtext package:

1. load_model: We loaded a model with pre-trained word vectors. The model was obtained from Wikipedia 2017, UMBC webbase corpus and statmt.org news dataset (MIKOLOV et al., 2017), which contains 1 million word vectors trained with sub-word information ${ }^{3}$.

2. get_sentence_representation: Then, we learned the vector representation of each document in our collection using the loaded model with pre-trained word vectors.

3. execute and predict: We created a trained model of the corpus for the classifier, where the following parameters were used: supervised, input $=$ file with labeled documents, $\operatorname{dim}=$ $100, l r=1$, wordNgrams $=2$, epoch $=20$, and output $=$ the trained model. From the model, it is possible to predict unlabeled documents.

4. execute and get_nn We also created an unsupervised model for our collection, with the following parameter settings: skipgram, input = union of documents from the collection, and output $=$ the unsupervised trained model. We applied the unsupervised model in order to retrieve $n$ words which vector representation is close to a selected one.

The system has been developed using d3.js ${ }^{4}$, a JavaScript library that supports the development of dynamic visualizations (BOSTOCK; OGIEVETSKY; HEER, 2011), and web 
technologies such as CSS, HTML and JavaScript. Developed to work on web browsers, the system is easily accessible and can be executed on personal data collections. The code is available ${ }^{5}$.

\subsection{Validation}

In order to validate the system and ensure it meets the actual needs of potential users, we had the collaboration of two professors in Physics, plus a Masters student in Computer Science who faced complex information retrieval problems as part of their research work. Informed about the goals and functionalities of TRIVIR, they showed interest in experimenting with the system. The two Physics professors are collaborating to conduct a broad literature survey on topics related to materials science research, for which formulating precise queries is particularly difficult. Indeed, the terms materials science and design cover a range of topics, with contributions expected from different fields. Thus the choice of search keywords is by no means straightforward. This was a strong motivation for them to engage on the validation effort of TRIVIR. The Masters student had conducted a systematic literature review, and welcomed an opportunity to verify his findings with an alternative approach. In particular, he was concerned about missing relevant publications.

An early validation effort was conducted with one of the Professors, which raised several issues and contributed to fine-tuning the visual interface. The later sessions, with the second Professor and the Masters student, were conducted using the current version of the system. The author of this dissertation participated in all sessions. During the sessions, the users were encouraged to report their insights about the system as well as suggestions for improvement.

\subsubsection{Preliminary Validation}

We conducted two early sessions with a potential user while the system was still under development. Our purpose was to verify usability issues and gather insights on how it could be improved to function properly in a realistic setting. Our collaborator had a collection of papers retrieved from the Web of Science (WoS) website using multiple search terms. Even though he was able to formulate an initial query, the search ended up retrieving a lot of documents not related to his topics of interest. This was mainly because contributions are expected from different fields, and the choice of keywords to search is by no means straightforward.

\subsubsection{Data and system setup}

The corpus used for the sessions was retrieved from the WoS website using the keywords (materials and design and discover, materials and design and discovery and (comput_or highthroughput or machine learning) and materials and design and discovery and (comput_or

5 https://github.com/amandagdias/TRIVIR 
high-throughput)). The results were merged into a collection of 2,095 papers, for which the abstract, title and the year of publication have been preserved. Regarding the system, we used the word embeddings representation of documents and the t-SNE projection technique to generate the scatterplot view.

\subsubsection{Results}

The Professor was really pleased with how easy the system was to learn. He explored mostly the Suggestion List, the Scatterplot and the Document views to filter out results. During the sessions, it became clear the need to filter the scatterplot in order to remove point clutter. Also, the Suggestion List view proved useful, as the returned documents were mostly considered relevant, even though the training set was small. However, the user wished to expand the sets of relevant/not relevant documents faster, labeling multiple documents at once. Besides, a single query document was not enough to represent his information needs. Indeed, in exploring the document similarity map he found important documents in different regions of the Scatterplot view. These usually corresponded to documents addressing distinct topics. Motivated by these issues we introduced three new functionalities into the system: (1) The ability to set multiple query documents. This will retrieve the 10 most similar documents to the new query and set them as relevant. (2) The ability to label as not relevant the 10 documents most similar to a not relevant document. (3) Setting as relevant/not relevant all documents containing a certain n-gram (in the Signature List view).

Even though functionalities (1) and (2) speed up the labeling process, they introduced an undesired aside effect, as irrelevant documents ended up included in the Focus List view, since new documents were added based solely on the cosine similarity distance to the selected document. In order to assign less importance to the documents added automatically, the relevant documents were assigned to three different classes, and the classifier results ranked based on the document's assigned class.

As mentioned, this collection was known to include many documents unrelated to the search, even though they employ the target keywords. The Professor considered these documents as "false positives". After the sessions were finished, we decided to investigate whether the Signature List view could help in reducing or eliminating what our collaborator called "the curse of false positives". First, we verified whether the information in the Signature List view could be used to filter out the false positives. We selected some frequent terms in the false positive documents labeled by the Professor and used them to filter the Signature List view to show only the 3-grams with those terms. We selected some 3-grams which we believed were common in the false positive documents, and filtered the corresponding documents. Then, we send a list containing those documents to the Professor and asked him to assess manually whether these were indeed false positives.

The Professor found that out of 101 documents identified, 82 were correctly labeled as not 
relevant. The relatively high number of documents mistakenly considered as not relevant might be explained by our lack of familiarity with the contents of the collection, which contributed to a poor selection of the representative 3-grams. From this experience, we realized that setting a group of documents as not relevant might result in the user missing important information if $\mathrm{s} / \mathrm{he}$ is not really certain that the 3-gram only appears in not relevant documents. Moreover, we found some bugs during the session that were fixed later and also introduced a few minor modifications in the interface to enhance usability.

\subsubsection{A literature review problem}

Following up the preliminary validation session, system development was finished fixing the issues identified and incorporating the suggestions. With the updates in place we moved on to perform further validation sessions on two real-case scenarios. The first is similar to the previous one, now involving the second Physics Professor, working on a different, though related, corpus.

\subsubsection{Data and system setup}

The second collection consisted of authors, abstract, year of publication and title information relative to 1,363 papers. Again, the corpus had been retrieved from WoS, but with slightly different search keywords: ((material* and (discovery or design) and ("machine learning" or "high-throughput" or "big data"))), applying a filter to restrict categories related to their research area. This time, rather than word embeddings, we decided to adopt the VSM document representation. We also modified the classifier learning rate from 1.0 (previous) to 0.1 .

\subsubsection{Results}

We observed that the scatterplot projection obtained using the VSM representation yielded better separation of the documents in the Scatterplot view than the word embeddings (Figure 37). It is possible to notice small groups of documents related with the same topic. However, exploring the map we identified some occurrences of the vocabulary mismatch issue where, in a group of mostly not relevant documents, the user found a few documents that were actually relevant. We realized that even though the papers share a target term, the context of usage is different. For example, the term "cell" appears in contexts of biology (e.g. cancer cells) and physics (e.g. solar cells).

The Professor searched the Signature List view for the term "cell" and retrieved the contexts on which it was used. By inspecting the documents in which the selected 3-grams occurred, he could set all of them as relevant or not relevant at once (Figure 38). Similar actions were performed on different terms, such as "machine learn model", "computational material design", "rational material design", and "lithium ion battery". 
Figure 37 - Scatterplot projections of a corpus with 1,363 papers, both created with the t-SNE projection technique and different document vector representations, namely word embeddings in (a); the VSM and a TF-IDF weighting scheme in (b). Blue circles correspond to documents currently labeled as relevant, yellow to documents suggested as relevant, not relevant documents are in red and the query documents in green.

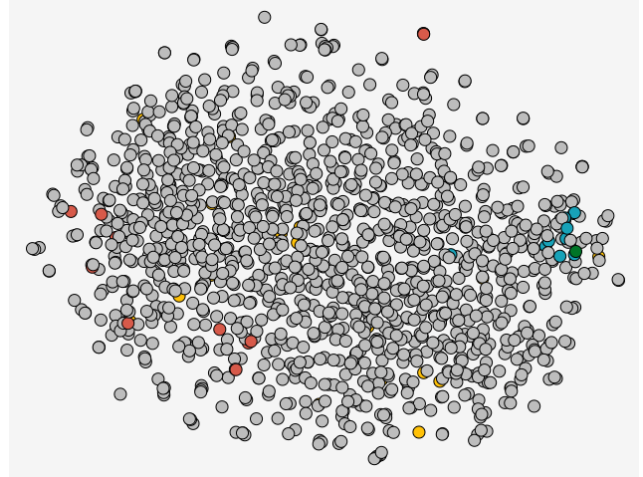

(a) Word Embedding

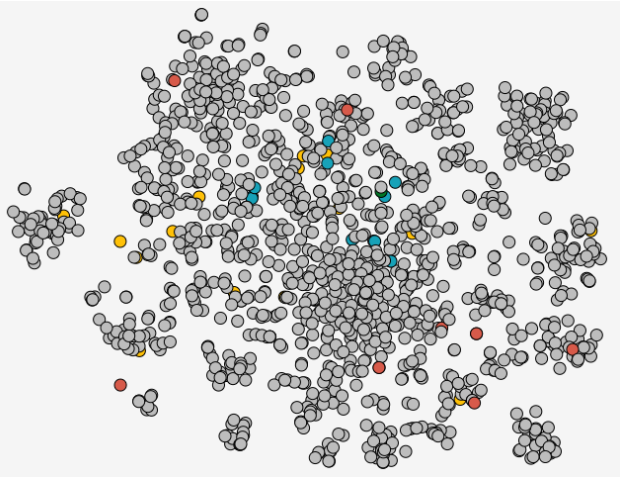

(b) VSM + TF-IDF

Developed by author.

Figure 38 - After searching for the word "cell" in the signature view, the user realized it is used in different contexts in the collection. Thus, he scanned the list and set all documents containing interesting 3-grams as relevant.

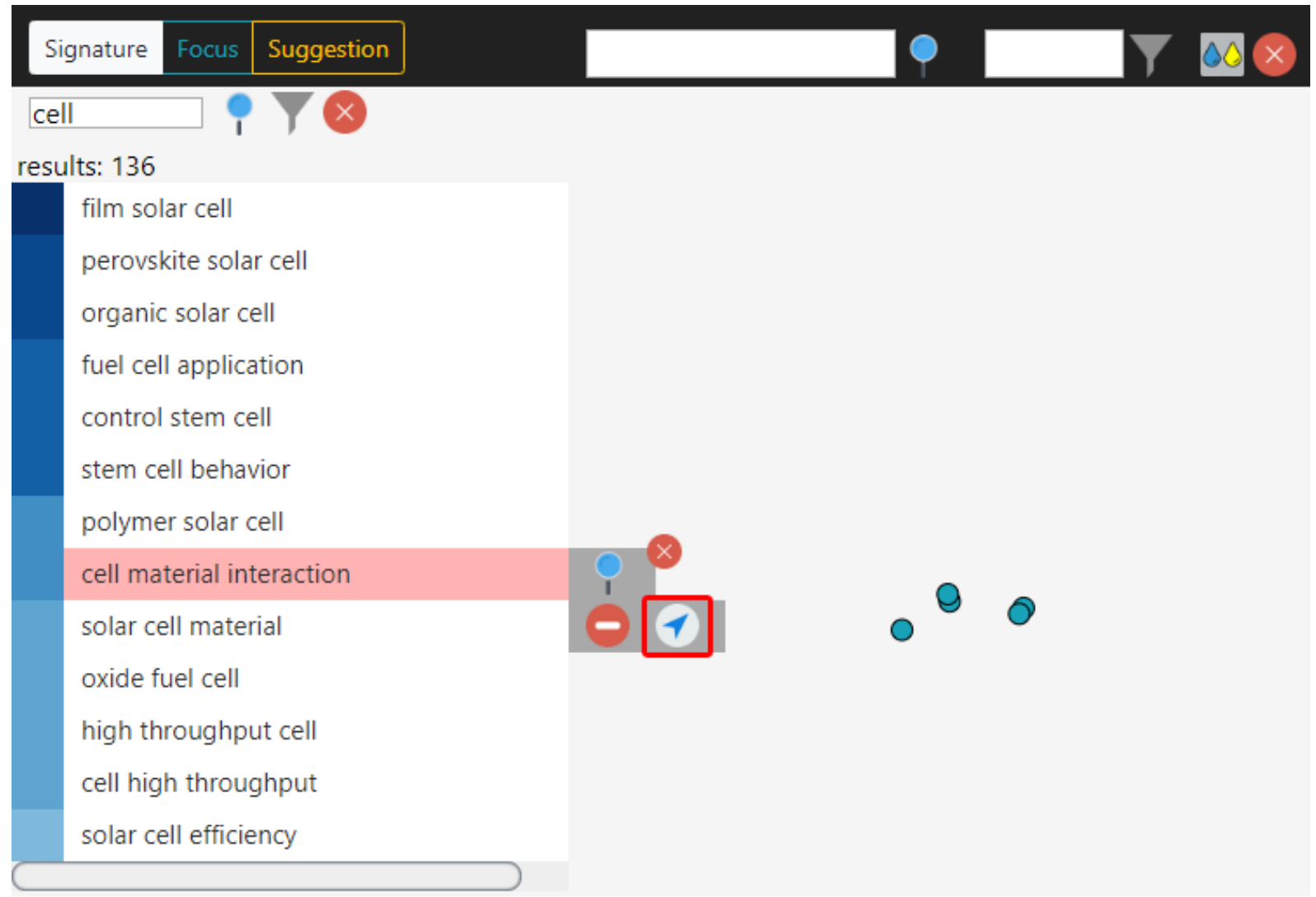

Developed by author. 
The menu on the Scatterplot view proved useful, since the Professor was able to visualize the relationships of documents containing certain author names and keywords. Also, by filtering the $\mathrm{K}$ documents most similar to the first query document, he realized the relevant documents were concentrated in the middle of the projection map, and did not belong to a well defined visual cluster.

We noticed a problem, however, which did not occur in the previous validation sessions, regarding the accuracy of the classifier output. Out of 20 documents included in the Suggestion list the Professor considered only 6 as actually relevant. Moreover, the 14 not relevant documents were clearly not related to the target themes. We conjectured this might be due to the change in the classifier learning rate. In a post-processing task conducted after the session we verified this assumption, setting the learning rate again to the previous value $(\mathrm{l} r=1)$ and re-executing the process, asking the Professor to assess the results again. With this new configuration, he considered 9 out of the 20 suggested documents as relevant. This result opens up the possibility of incorporating and testing different ML algorithms and configurations, in order to investigate how results can be improved.

Overall, the professor was pleased with the visualization and was able to retrieve over 200 relevant documents in a session that lasted under two hours. Most of the selection effort was conducted in the final 30 minutes, after he decided to use the functionalities of the Signature List view.

\subsubsection{A Systematic Mapping Study (SMS) scenario}

Our third collaborator is a Master's student who had conducted a Systematic Mapping Study to identify all relevant studies related to his research. His is interested in papers describing systems to teach Computer Science concepts through Virtual Reality (VR), Augmented Reality (AR) or Mixed Reality (MR), specially those developed for mobile devices. After manually reviewing a large collection of papers he identified 15 as relevant to his research. He wanted to use TRIVIR to check whether he might have missed important papers and thus improve the results of his SMS. We were particularly interested in comparing the sets of papers obtained with the manual approach and using TRIVIR.

\subsubsection{Data and system setup}

The student used the parsif. $\mathrm{al}^{6}$ tool in order to support his SMS and retrieved 1,259 papers from the ACM, Engineering Village, IEEE and Scopus data bases. The provided search string was ("learning" OR "teaching") AND ("programming") AND ( "augmented reality" OR "virtual reality" $O R$ "mixed reality"). We were able to download a .csv file from parsif.al containing all the papers and upload to our system the information of title, authors, year, abstract and keywords.

6 https://parsif.al/ 
We set the t-SNE as the projection technique and the VSM using the TF-IDF weighting scheme as document representation.

\subsubsection{Results}

The student started interacting with the Suggestion List view and considered relevant only 1 out of the 20 documents. It is important to point out, however, that he already had a in-depth knowledge of the corpus. In fact, some papers now directly labeled as not relevant had been considered for inspection in the SMS effort, and discarded only after reading.

He observed that several papers included relevant keywords, such as "augmented reality" and "virtual reality", but their reported contribution did not actually focus on these concepts. Similarly, the keywords of some relevant studies were not actually representative of their content, and thus misleading.

The student removed 5 documents from the initial Focus List view, which brought some not relevant papers. He found these papers, even though they had some similarities with the query document, did not meet his needs as they were not specific enough. The selected query document appeared in the Scatterplot view within a small cluster containing relevant documents. He investigated this group and marked 3 additional documents as relevant.

As he continued exploring the Scatterplot view, he encountered some papers on games, a topic he considered not related to his interest, even though it is slightly related to his search. Then, intending to remove documents that included this term, he searched in the Signature List view and found the 3-gram "game base learn". Surprisingly, there were relevant papers that used this 3-gram and, instead of removing any documents with this particular 3-gram he actually identified 4 additional relevant papers.

This motivated him to search for additional 3-grams, where he found additional relevant papers containing the 3-grams "virtual reality vr", "augmented reality ar", "augmented reality learn" and, "virtual reality virtual", including papers not identified as relevant in his manual review. After he finished exploring the Signature List and the Scatterplot views, he retrained the ML algorithm, however, none of the suggested results were relevant. He decided to retrain the algorithm again and found one more relevant document. After a final training iteration where no additional relevant document was suggested the student decided to stop the session to verify the results. The entire session took slightly under 2 hours.

The final Focus List view included 26 documents and their distribution is illustrated in Figure 39, which we compared against the previous set of relevant papers obtained in the SMS. With TRIVIR the student retrieved 11 of the 15 papers previously found, and 15 new potentially relevant papers. We then tried to understand the reasons for missing 4 papers from the original SMS set. A common issue to all papers was that their keywords were not specific, so as to help filtering them. Moreover, two of the papers were marginally relevant, did not share the same 
keywords and their abstracts were missing. However, one of the missed papers included all the information required to be easily identified in the visualization. After some analysis we found out that it included the 3-gram "game base introduction", not contained in the Signature List view, even though the user searched for the 3-gram "game base learn". The reason is that this 3-gram "game base introduction" occurred only once and thus was not included in the list. Besides, the distribution of relevant documents found by the users in all three validation exercises suggested that documents of interest might not be delimited by a specific cluster.

Figure 39 - Distribution of labeled documents at the end of the SMS session.

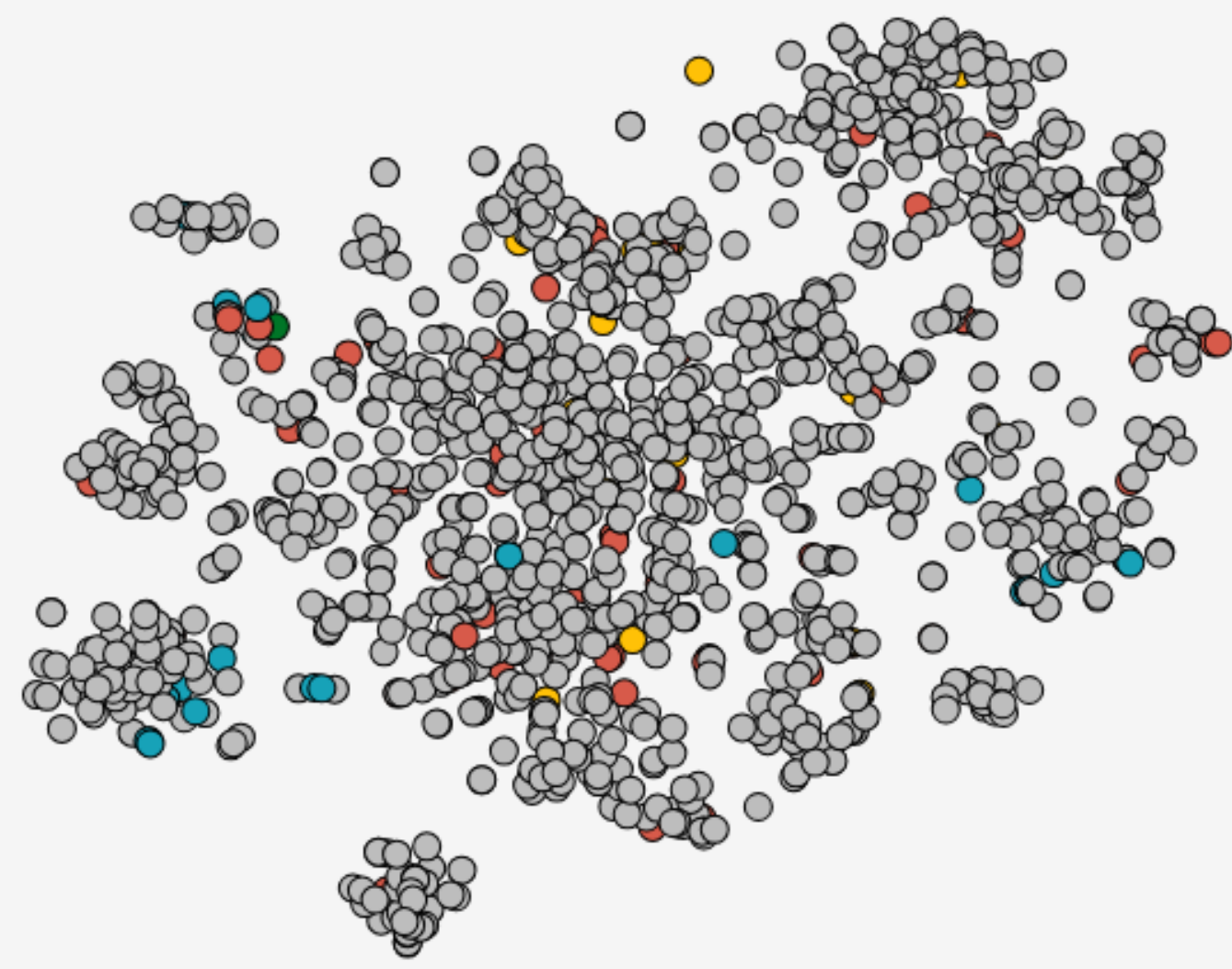

Developed by author.

In conclusion, the user made a positive assessment of the system, as it allowed him to expand the set of representative papers. These results suggest that TRIVIR can also be useful to verify results of manual literature review efforts. We also identified some problems regarding the information shown in the Signature List view, mainly that 3-grams with frequency 1 may be relevant to a search and should not be discarded, despite the risk of introducing scalability issues. The student also gave us useful insights about possible changes in the interface design that may enhance the user experience. 


\subsection{Final considerations}

In this Chapter we introduced TRIVIR, a novel visualization system to support document retrieval with high recall. More specifically, the presented system offers the following possibilities:

- Explore a document collection projected as a content similarity map to show documents' relationships, query included.

- Label documents as relevant, not relevant or query.

- Easily expand the set of relevant and not relevant documents considering the most similar documents to a selected one.

- Identify the context on which terms appear in documents, with the option to set all documents containing a specific 3-gram as relevant or not relevant.

- Identify the synonyms of keywords and discover new terms that are used in similar contexts.

- Explore a list of suggested documents created by a machine learning algorithm.

The validation exercises suggested the system supported users in performing the described tasks and was well accepted by them. As next steps, we consider modify the current environment to become more user friendly and make the system easily accessible through an open website. We also observed that each view of the system could be enhanced in different ways to further improve the user experience. For example, the Scatterplot view could group documents using a clustering algorithm, the Document view could highlight important terms and sections of the text, and the Signature view could present the frequency and distribution of 3-grams on selected documents. Regarding the underlining techniques, the system is flexible to deal with different methods which allows studies comparing different document representations and classification algorithms for document retrieval along with text visualization. 
CHAPTER

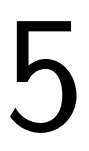

\section{CONCLUSIONS}

In this work we developed TRIVIR, a system that combines IR with interactive visualization techniques in order to enhance document retrieval. Users can interact with the ML model to retrieve relevant documents from a collection. The system applies the CAL protocol, which trains a classification algorithm based on user's relevance feedback. Whilst a standard approach shows to users a ranked list where each document must be inspected individually, in TRIVIR users sees the suggested documents in relation to others, can explore other documents than those suggested by the classifier, can inspect the context in which terms occur in the documents and can label several documents at once, speeding up the process. A user starts selecting a query document representative of her information needs. S/he can add query documents in the process and select the document representation and the projection technique to be used.

Source code is available online ${ }^{1}$ and it was developed to be flexible in terms of document representation, multidimensional projection and classification techniques, which implies that the original code can be easily enhanced with additional approaches. Moreover, TRIVIR was built considering that it will run in a public server where users can access it through a public web page. A paper with results from this project was submitted to the $19^{\text {th }}$ ACM Symposium on Document Engineering ${ }^{2}$.

We validated TRIVIR with the collaboration of two Physics professors and a Master's student, who used the system in real case scenarios and expressed positive impressions of TRIVIR. The system was considered user-friendly, and the users learnt how to navigate and explore its functions within minutes after a brief explanation. The Signature List view was the only one that demanded some additional effort from users. Still, it proved a key feature to label groups of documents and speed up the process.

The usefulness of TRIVIR is not so easy to demonstrate. In order to benefit from TRIVIR

1 https://github.com/amandagdias/TRIVIR

2 https://doceng.org/doceng2019/ 
users must be prepared to explore different strategies, which may not be easy for those with limited experience with semi-automated surveys or computational tools to assist in surveys. A likely future development of TRIVIR could be in creating a tutorial functionality to assist users in understanding different strategies and how they can be combined to achieve their goal.

In the validation effort we identified that users may end up finding relevant documents within the documents labeled as "not relevant". This usually happened after employing the functionality to label the 10 most similar document to a selected one as "not relevant". This problem is similar to the one we faced when selecting additional query documents. However, labeling relevant documents as "not relevant" negatively impacts our purpose of achieving high recall. Thus, even though it quickly enlarges the set of not relevant documents, this functionality must be employed with caution. Moreover, removing low frequent 3-grams may also negatively affect the search for important documents in certain scenarios.

Another limitation is scalability. In general, our experiments used a collection of around 2,500 documents and the system took about 10 minutes to load all the required resources and approximately 800 megabytes of data was generated. Even though users could load more documents, they would have to consider the time and memory necessary. In order to handle the increase in the size of the data, a modification in TRIVIR's Scatterplot View might be necessary since the clutter would prevent the user from properly inspecting the results. A solution might be to apply a cluster technique on the data where similar data points are grouped together and more details can be given on demand.

Nevertheless, TRIVIR enables information retrieval tasks considering not only suggested documents from a ML algorithm but it also incorporates visualizations that guides users in retrieving important documents from a collection. Additionally, the vocabulary-mismatch issue was handled by providing words context through the Signature View and synonyms through the Terms View.

Possible applications for TRIVIR include:

- Support information retrieval tasks with high recall. Examples include bibliographic research, but the system is not restricted to a particular application.

- Validate results obtained in previous retrieval tasks, such as a Systematic Mapping Study.

- Compare different document representation, multidimensional projection, classification and similarity computation techniques.

- Perform exploratory tasks in document collections in general.

Even though our validation exercises provided valuable feedback and suggested that TRIVIR was useful for the proposed tasks, we believe that a formal evaluation with users in 
distinct domains applications may provide more solid evidence of the system's effectiveness and potential.

Possible future lines of research include:

- Expand techniques options for document representation, multidimensional projection, similarity computation and text classification.

- Apply concepts from Human Computer Interaction in order to assess and improve the system's usability and make it easier to end users.

- Incorporate new functionalities into some of TRIVIR's main views, such as: (1) cluster data points in the Scatterplot view; (2) update the machine learning model considering the Terms' view interactions, and (3) create signatures for each document. 

BAEZA-YATES, R. A.; RIBEIRO-NETO, B. Modern Information Retrieval. Boston, MA, USA: Addison-Wesley Longman Publishing Co., Inc., 1999. ISBN 020139829X. Citation on page 30 .

BERENDT, B.; SUBASIC, I. Stories in time: A graph-based interface for news tracking and discovery. In: Proceedings of the 2009 IEEE/WIC/ACM International Joint Conference on Web Intelligence and Intelligent Agent Technology - Volume 03. IEEE Computer Society, 2009. (WI-IAT '09), p. 531-534. ISBN 978-0-7695-3801-3. Available: <http://dx.doi.org/10. 1109/WI-IAT.2009.342>. Citations on pages 48 and 50.

BERGER, M.; MCDONOUGH, K.; SEVERSKY, L. M. cite2vec: Citation-driven document exploration via word embeddings. IEEE Transactions on Visualization and Computer Graphics, v. 23, n. 1, p. 691-700, Jan 2017. ISSN 1077-2626. Citations on pages 48 and 51.

BLAIR, D. C.; MARON, M. E. An evaluation of retrieval effectiveness for a full-text documentretrieval system. Communications of the ACM, ACM, v. 28, n. 3, p. 289-299, Mar. 1985. ISSN 0001-0782. Available: <http://doi.acm.org/10.1145/3166.3197>. Citations on pages 23, 27, and 30.

BOJANOWSKI, P.; GRAVE, E.; JOULIN, A.; MIKOLOV, T. Enriching word vectors with subword information. Transactions of the Association for Computational Linguistics, v. 5, p. 135-146, 2017. Citations on pages 34, 70, 71, and 74.

BOSTOCK, M.; OGIEVETSKY, V.; HEER, J. D3 data-driven documents. IEEE Transactions on Visualization and Computer Graphics, IEEE Educational Activities Department, v. 17, n. 12, p. 2301-2309, Dec. 2011. ISSN 1077-2626. Available: <http://dx.doi.org/10.1109/TVCG. 2011.185>. Citation on page 76 .

BRADEL, L.; NORTH, C.; HOUSE, L.; LEMAN, S. Multi-model semantic interaction for text analytics. In: 2014 IEEE Conference on Visual Analytics Science and Technology (VAST). Paris, France: IEEE, 2014. p. 163-172. Citations on pages 61, 62, and 64.

BYRD, D. A scrollbar-based visualization for document navigation. In: Proceedings of the Fourth ACM Conference on Digital Libraries. ACM, 1999. (DL '99), p. 122-129. ISBN 1-58113-145-3. Available: <http://doi.acm.org/10.1145/313238.313283>. Citation on page 45.

CAO, N.; SUN, J.; LIN, Y.; GOTZ, D.; LIU, S.; QU, H. Facetatlas: Multifaceted visualization for rich text corpora. IEEE Transactions on Visualization and Computer Graphics, v. 16, n. 6, p. 1172-1181, Nov 2010. ISSN 1077-2626. Citations on pages 55, 58, and 64.

CHALMERS, M.; CHITSON, P. Bead: Explorations in information visualization. In: Proceedings of the 15th Annual International ACM SIGIR Conference on Research and Development in Information Retrieval. ACM, 1992. (SIGIR '92), p. 330-337. ISBN 0-89791-523-2. Available: <http://doi.acm.org/10.1145/133160.133215>. Citations on pages 54 and 55. 
CHEN, C. Citespace ii: Detecting and visualizing emerging trends and transient patterns in scientific literature. J. Am. Soc. Inf. Sci. Technol., John Wiley \& Sons, Inc., v. 57, n. 3, p. 359377, Feb. 2006. ISSN 1532-2882. Available: <http://dx.doi.org/10.1002/asi.v57:3>. Citations on pages 54,56 , and 58 .

CHOO, J.; KIM, H.; CLARKSON, E.; LIU, Z.; LEE, C.; LI, F.; LEE, H.; KANNAN, R.; STOLPER, C. D.; STASKO, J.; PARK, H. Visirr: A visual analytics system for information retrieval and recommendation for large-scale document data. ACM Transactions on Knowledge Discovery from Data, ACM, v. 12, n. 1, p. 8:1-8:20, Jan. 2018. ISSN 1556-4681. Available: $<$ http://doi.acm.org/10.1145/3070616>. Citations on pages 50, 52, and 64 .

CHOU, J.-K.; YANG, C.-K. Papervis: Literature review made easy. In: Proceedings of the 13th Eurographics / IEEE - VGTC Conference on Visualization. Chichester, UK: The Eurographs Association; John Wiley; Sons, Ltd., 2011. (EuroVis'11), p. 721-730. Available: <http://dx.doi. org/10.1111/j.1467-8659.2011.01921.x>. Citation on page 55 .

CORMACK, G. V.; GROSSMAN, M. R. The Grossman-Cormack Glossary of Technology Assisted Review. 2013. Available: <http://www.fclr.org/fclr/articles/html/2010/grossman.pdf>. Accessed: 27/07/2017. Citations on pages 24 and 29.

Evaluation of machine-learning protocols for technology-assisted review in electronic discovery. In: Proceedings of the 37th International ACM SIGIR Conference on Research; Development in Information Retrieval. ACM, 2014. (SIGIR '14), p. 153-162. ISBN 978-14503-2257-7. Available: <http://doi.acm.org/10.1145/2600428.2609601>. Citations on pages 25 and 29.

Engineering quality and reliability in technology-assisted review. In: Proceedings of the 39th International ACM SIGIR Conference on Research and Development in Information Retrieval. ACM, 2016. (SIGIR '16), p. 75-84. ISBN 978-1-4503-4069-4. Available: <http: //doi.acm.org/10.1145/2911451.2911510>. Citation on page 29.

A tour of technology-assisted review. In: FAGERBERG, J.; MOWERY, D. C.; NELSON, R. R. (Ed.). Perspectives on Predictive Coding and Other Advanced Search Methods for the Legal Practitioner. [S.1.]: American Bar Association, 2016. chap. 3. ISBN 978-1-63425657-5. Citation on page 29.

CROFT, B.; METZLER, D.; STROHMAN, T. Search Engines: Information Retrieval in Practice. 1st. ed. USA: Addison-Wesley Publishing Company, 2009. ISBN 0136072240, 9780136072249. Citations on pages 28 and 30.

CUTTING, D. R.; KARGER, D. R.; PEDERSEN, J. O.; TUKEY, J. W. Scatter/gather: A cluster-based approach to browsing large document collections. In: Proceedings of the 15th Annual International ACM SIGIR Conference on Research and Development in Information Retrieval. ACM, 1992. (SIGIR '92), p. 318-329. ISBN 0-89791-523-2. Available: $<$ http://doi.acm.org/10.1145/133160.133214>. Citations on pages 47 and 48.

DUNNE, C.; SHNEIDERMAN, B.; GOVE, R.; KLAVANS, J.; DORR, B. Rapid understanding of scientific paper collections: Integrating statistics, text analytics, and visualization. Journal of the American Society for Information Science and Technology, v. 63, n. 12, p. 2351-2369, 2012. Available: <https://onlinelibrary.wiley.com/doi/abs/10.1002/asi.22652>. Citation on page 55 . 
ENDERT, A.; BURTNER, R.; CRAMER, N.; PERKO, R.; HAMPTON, S.; COOK, K. Typograph: Multiscale spatial exploration of text documents. In: 2013 IEEE International Conference on Big Data. Silicon Valley, CA, USA: IEEE, 2013. p. 17-24. Citations on pages 53 and 54.

ENDERT, A.; FIAUX, P.; NORTH, C. Semantic interaction for visual text analytics. In: Proceedings of the SIGCHI Conference on Human Factors in Computing Systems. ACM, 2012. (CHI '12), p. 473-482. ISBN 978-1-4503-1015-4. Available: <http://doi.acm.org/10. 1145/2207676.2207741>. Citations on pages 61 and 62.

FELIZARDO, K. R.; ANDERY, G. F.; PAULOVICH, F. V.; MINGHIM, R.; MALDONADO, J. C. A visual analysis approach to validate the selection review of primary studies in systematic reviews. Information and Software Technology, v. 54, n. 10, p. 1079 - 1091, 2012. ISSN 0950-5849. Available: <http://www.sciencedirect.com/science/article/pii/S0950584912000742>. Citations on pages 54 and 57.

FRUCHTERMAN, T. M. J.; REINGOLD, E. M. Graph drawing by force-directed placement. Software: Practice and Experience, John Wiley \& Sons, Inc., v. 21, n. 11, p. 1129-1164, Nov. 1991. ISSN 0038-0644. Available: <http://dx.doi.org/10.1002/spe.4380211102>. Citation on page 39.

GOMEZ-NIETO, E.; ROMAN, F. S.; PAGLIOSA, P.; CASACA, W.; HELOU, E. S.; OLIVEIRA, M. C. F.; NONATO, L. G. Similarity preserving snippet-based visualization of web search results. IEEE Transactions on Visualization and Computer Graphics, IEEE Educational Activities Department, v. 20, n. 3, p. 457-470, Mar. 2014. ISSN 1077-2626. Available: <http: //dx.doi.org/10.1109/TVCG.2013.242>. Citations on pages 60 and 64.

GRANITZER, M.; KIENREICH, W.; SABOL, V.; DöSINGER, G. Webrat: Supporting agile knowledge retrieval through dynamic, incremental clustering and automatic labelling of web search result sets. In: Proceedings of the Twelfth International Workshop on Enabling Technologies: Infrastructure for Collaborative Enterprises. Washington, DC, USA: IEEE Computer Society, 2003. (WETICE '03), p. 296-. ISBN 0-7695-1963-6. Available: $<$ http://dl.acm.org/citation.cfm?id=938984.939786>. Citations on pages 47 and 49.

GROSSMAN, M.; CORMACK, G. Continuous active learning for tar. Practical Law The Journal: Litigation, p. 32-37, Apr-May 2016. Citations on pages 29 and 75.

GROSSMAN, M. R.; CORMACK, G. V. Technology-Assisted Review in E-Discovery Can Be More Effective and More Efficient Than Exhaustive Manual Review. Richmond Journal of Law and Technology, XVII, n. 3, p. 1-33, 2011. ISSN 02729490. Available: <http://www. discovia.com/wp-content/uploads/2012/07/grossman-cormack-20110326.pdf>. Citation on page 24 .

GöRG, C.; LIU, Z.; KIHM, J.; CHOO, J.; PARK, H.; STASKO, J. Combining computational analyses and interactive visualization for document exploration and sensemaking in jigsaw. IEEE Transactions on Visualization and Computer Graphics, v. 19, n. 10, p. 1646-1663, Oct 2013. ISSN 1077-2626. Citation on page 57.

HARPER, D. J.; COULTHARD, S.; YIXING, S. A language modelling approach to relevance profiling for document browsing. In: Proceedings of the 2Nd ACM/IEEE-CS Joint Conference on Digital Libraries. ACM, 2002. (JCDL '02), p. 76-83. ISBN 1-58113-513-0. Available: $<$ http://doi.acm.org/10.1145/544220.544234>. Citation on page 45. 
HEARST, M. A. Tilebars: Visualization of term distribution information in full text information access. In: Proceedings of the SIGCHI Conference on Human Factors in Computing Systems. ACM Press/Addison-Wesley Publishing Co., 1995. (CHI '95), p. 59-66. ISBN 0201-84705-1. Available: <http://dx.doi.org/10.1145/223904.223912>. Citations on pages 45 and 46.

HEIMERL, F.; JOHN, M.; HAN, Q.; KOCH, S.; ERTL, T. Docucompass: Effective exploration of document landscapes. In: 2016 IEEE Conference on Visual Analytics Science and Technology (VAST). Baltimore, MD, USA: IEEE, 2016. p. 11-20. Citations on pages 57, 59, and 64 .

HINTON, G.; ROWEIS, S. Stochastic neighbor embedding. In: Proceedings of the 15th International Conference on Neural Information Processing Systems. MIT Press, 2002. (NIPS'02), p. 857-864. Available: <http://dl.acm.org/citation.cfm?id=2968618.2968725>. Citation on page 39.

INGRAM, S.; MUNZNER, T. Dimensionality reduction for documents with nearest neighbor queries. Neurocomputing, v. 150, 11 2014. Citation on page 40.

JANKOWSKA, M.; KESELJ, V.; MILIOS, E. Relative n-gram signatures: Document visualization at the level of character n-grams. In: Proceedings of the 2012 IEEE Conference on Visual Analytics Science and Technology (VAST). Washington, DC, USA: IEEE Computer Society, 2012. (VAST '12), p. 103-112. ISBN 978-1-4673-4752-5. Available: $<$ http://dx.doi.org/10.1109/VAST.2012.6400484>. Citation on page 71.

JONES, K. S. Document retrieval systems. In: WILLETT, P. (Ed.). London, UK, UK: Taylor Graham Publishing, 1988. chap. A Statistical Interpretation of Term Specificity and Its Application in Retrieval, p. 132-142. ISBN 0-947568-21-2. Available: <http://dl.acm.org/citation.cfm? $\mathrm{id}=106765.106782>$. Citations on pages 32 and 74 .

JOULIN, A.; GRAVE, E.; BOJANOWSKI, P.; MIKOLOV, T. Bag of tricks for efficient text classification. In: Proceedings of the 15th Conference of the European Chapter of the Association for Computational Linguistics. Association for Computational Linguistics, 2017. p. 427-431. Available: <http://aclweb.org/anthology/E17-2068>. Citations on pages 37, 70, and 75 .

KADHIM, A. Survey on supervised machine learning techniques for automatic text classification. Artificial Intelligence Review, 01 2019. Citation on page 37.

KAMATH, C. N.; BUKHARI, S. S.; DENGEL, A. Comparative study between traditional machine learning and deep learning approaches for text classification. In: Proceedings of the ACM Symposium on Document Engineering 2018. ACM, 2018. (DocEng '18), p. 14:114:11. ISBN 978-1-4503-5769-2. Available: <http://doi.acm.org/10.1145/3209280.3209526>. Citations on pages 37 and 38 .

KAMPANYA, N.; SHEN, R.; KIM, S.; NORTH, C.; FOX, E. Citiviz: A visual user interface to the CITIDEL system. In: Heery R., Lyon L. (eds) Research and Advanced Technology for Digital Libraries. ECDL, 2004. p. 122-133. Available: <https://doi.org/10.1007/ 978-3-540-30230-8_12>. Citation on page 59.

KEIM, D.; ANDRIENKO, G.; FEKETE, J.-D.; GÖRG, C.; KOHLHAMMER, J.; MELANÇON, G. Visual analytics: Definition, process, and challenges. Information visualization, Springer, p. 154-175, 2008. Citation on page 23. 
KIELA, D.; HILL, F.; CLARK, S. Specializing word embeddings for similarity or relatedness. In: Proceedings of the 2015 Conference on Empirical Methods in Natural Language Processing. Association for Computational Linguistics, 2015. p. 2044-2048. Available: $<$ http://aclweb.org/anthology/D15-1242>. Citation on page 36.

KLOUCHE, K.; RUOTSALO, T.; MICALLEF, L.; ANDOLINA, S.; JACUCCI, G. Visual re-ranking for multi-aspect information retrieval. In: Proceedings of the 2017 Conference on Conference Human Information Interaction and Retrieval. ACM, 2017. (CHIIR '17), p. 57-66. ISBN 978-1-4503-4677-1. Available: <http://doi.acm.org/10.1145/3020165.3020174>. Citations on pages 44 and 45.

KOCH, S.; HEIMERL, F.; ERTL, T. Visual document retrieval: Supporting text search and analysis with visual analytics. Computing in Science Engineering, v. 15, n. 4, p. 66-74, JulyAug. 2013. ISSN 1521-9615. Available: <doi.ieeecomputersociety.org/10.1109/MCSE.2013.93>. Citation on page 37.

KORHONEN, A.; LITOLA, J.; TARHIO, J. Platform for elaboration of search results. Webist 2007 - 3rd International Conference on Web Information Systems and Technologies, Proceedings, p. 263-269, 01 2007. Citation on page 47.

KUMARI, M.; JAIN, A.; BHATIA, A. Synonyms based term weighting scheme: An extension to tf.idf. Procedia Computer Science, v. 89, p. 555 - 561", 2016. ISSN 1877-0509. Available: $<$ http://www.sciencedirect.com/science/article/pii/S1877050916311589>. Citation on page 32.

LE, Q.; MIKOLOV, T. Distributed representations of sentences and documents. In: Proceedings of the 31st International Conference on International Conference on Machine Learning Volume 32. JMLR.org, 2014. (ICML'14), p. II-1188-II-1196. Available: <http://dl.acm.org/ citation.cfm?id=3044805.3045025> . Citations on pages 35 and 36 .

LIU, S.; WANG, X.; COLLINS, C.; DOU, W.; OUYANG, F.; M, E. A.; JIANG, L.; KEIM, D. Bridging text visualization and mining: A task-driven survey. IEEE Transactions on Visualization and Computer Graphics, p. 1-1, 2018. ISSN 1077-2626. Citation on page 63.

MAATEN, L. Accelerating t-sne using tree-based algorithms. Journal of Machine Learning Research, v. 15, p. 3221-3245, 2014. Available: <http://jmlr.org/papers/v15/vandermaaten14a. html>. Citation on page 74 .

MAATEN, L. V. D. Accelerating t-sne using tree-based algorithms. J. Mach. Learn. Res., JMLR.org, v. 15, n. 1, p. 3221-3245, Jan. 2014. ISSN 1532-4435. Available: <http://dl.acm.org/ citation.cfm?id=2627435.2697068 $>$. Citation on page 40 .

MAATEN, L. V. D.; POSTMA, E.; HERIK, J. Van den. Dimensionality reduction: a comparative review. J Mach Learn Res, v. 10, p. 66-71, 2009. Citations on pages 38 and 39.

MAATEN, L. van der; HINTON, G. Visualizing data using t-SNE. Journal of Machine Learning Research, v. 9, p. 2579-2605, 2008. Available: <http://www.jmlr.org/papers/v9/ vandermaaten08a.html>. Citations on pages 39 and 40 .

MAKKI, R.; CARVALHO, E.; SOTO, A. J.; BROOKS, S.; OLIVEIRA, M. C. F. de; MILIOS, E.; MINGHIM, R. Atr-vis: Visual and interactive information retrieval for parliamentary discussions in twitter. To appear in ACM Transactions on Knowledge Discovery from Data, 2017. Citation on page 24 . 
MANNING, C. D.; RAGHAVAN, P.; SCHüTZE, H. Introduction to Information Retrieval. [S.1.]: Cambridge University Press, 2008. ISBN 0521865719, 9780521865715. Citations on pages 30,31 , and 32 .

MIKOLOV, T.; CORRADO, G.; CHEN, K.; DEAN, J. Efficient estimation of word representations in vector space. In: . [S.1.]: Computing Research Repository (CoRR), 2013. p. 1-12. Citations on pages 33 and 34 .

MIKOLOV, T.; GRAVE, E.; BOJANOWSKI, P.; PUHRSCH, C.; JOULIN, A. Advances in pre-training distributed word representations. Computing Research Repository (CoRR), abs/1712.09405, 2017. Available: <http://arxiv.org/abs/1712.09405>. Citation on page 76.

MIKOLOV, T.; SUTSKEVER, I.; CHEN, K.; CORRADO, G.; DEAN, J. Distributed representations of words and phrases and their compositionality. In: Proceedings of the 26th International Conference on Neural Information Processing Systems. USA: Curran Associates Inc., 2013. (NIPS'13), p. 3111-3119. Available: <http://dl.acm.org/citation.cfm?id=2999792.2999959>. Citations on pages 33 and 34.

MIKOLOV, T.; YIH, S. W.-t.; ZWEIG, G. Linguistic regularities in continuous space word representations. In: Proceedings of the 2013 Conference of the North American Chapter of the Association for Computational Linguistics: Human Language Technologies (NAACL-HLT-2013). Association for Computational Linguistics, 2013. Available: <https://www.microsoft.com/en-us/research/publication/ linguistic-regularities-in-continuous-space-word-representations $/>$. Citation on page 34.

NGUYEN, T. N.; ZHANG, J. A novel visualization model for web search results. IEEE transactions on visualization and computer graphics, v. 12, p. 981-8, 09 2006. Citations on pages 46,47 , and 64 .

OLIVEIRA, M. C. F.; LEVKOWITZ, H. From visual data exploration to visual data mining: a survey. IEEE Transactions on Visualization and Computer Graphics, v. 9, n. 3, p. 378-394, 2003. Citation on page 23.

OLSEN, K. A.; KORFHAGE, R. R.; SOCHATS, K. M.; SPRING, M. B.; WILLIAMS, J. G. Visualization of a document collection: The vibe system. Information Processing \& Management, v. 29, n. 1, p. 69 - 81, 1993. ISSN 0306-4573. Available: <http://www.sciencedirect.com/ science/article/pii/0306457393900248>. Citations on pages 43 and 44.

PAIVA, J. G. S.; SCHWARTZ, W. R.; PEDRINI, H.; MINGHIM, R. An approach to supporting incremental visual data classification. IEEE Transactions on Visualization and Computer Graphics, v. 21, p. 4-17, 2015. Citation on page 37.

PAULOVICH, F.; NONATO, L.; MINGHIM, R.; LEVKOWITZ, H. Least square projection: A fast high-precision multidimensional projection technique and its application to document mapping. IEEE Transactions on Visualization and Computer Graphics, v. 14, n. 3, p. 564575, May-June 2008. ISSN 1077-2626. Citations on pages 40 and 74.

PAULOVICH, F. V.; MINGHIM, R. HiPP: A novel hierarchical point placement strategy and its application to the exploration of document collections. IEEE Transactions on Visualization and Computer Graphics, IEEE Educational Activities Department, Piscataway, NJ, EUA, v. 14, n. 6, p. 1229-1236, Nov. 2008. ISSN 1077-2626. Citation on page 41. 
PAULOVICH, F. V.; PINHO, R.; BOTHA, C. P.; HEIJS, A.; MINGHIM, R. Pex-web: Contentbased visualization of web search results. 2008 International Conference Information Visualisation, p. 208-214, July 2008. ISSN 1550-6037. Citations on pages 59, 61, and 64.

PEARSON, K. On lines and planes of closest fit to systems of points in space. Philosophical Magazine, v. 2, p. 559-572, 1901. Citation on page 38.

PEZZOTTI, N.; LELIEVELDT, B.; MAATEN, L. van der; HöLLT, T.; EISEMANN, E.; VILANOVA, A. Approximated and user steerable tsne for progressive visual analytics. IEEE Transactions on Visualization and Computer Graphics, v. 23, 07 2016. Citation on page 40.

RENNISON, E. Galaxy of news: An approach to visualizing and understanding expansive news landscapes. In: Proceedings of the 7th Annual ACM Symposium on User Interface Software and Technology. ACM, 1994. (UIST '94), p. 3-12. ISBN 0-89791-657-3. Available: $<$ http://doi.acm.org/10.1145/192426.192429>. Citations on pages 52, 53, and 64.

RINKER, T. W. qdap: Quantitative Discourse Analysis Package. Buffalo, New York, 2019. 2.3.2. Available: <http://github.com/trinker/qdap>. Citation on page 71 .

RUBENS, N.; KAPLAN, D.; SUGIYAMA, M. Active learning in recommender systems. Recommender Systems Handbook, Springer, p. 735-767, 2011. Citation on page 29.

SEDLMAIR, M.; MUNZNER, T.; TORY, M. Empirical guidance on scatterplot and dimension reduction technique choices. IEEE Transactions on Visualization and Computer Graphics, IEEE Educational Activities Department, Piscataway, NJ, USA, v. 19, n. 12, p. 2634-2643, Dec. 2013. ISSN 1077-2626. Available: <http://dx.doi.org/10.1109/TVCG.2013.153>. Citations on pages 41 and 68 .

SETTLES, B. Active learning literature survey. Computer Sciences Technical Report, n. 1648, 2009. Citation on page 28.

SINGHAL, A. Modern information retrieval: A brief overview. IEEE Data Engineering Bulletin, v. 24, n. 4, p. 35-43, 01 2001. Citations on pages 28 and 30.

STASKO, J.; GORG, C.; LIU, Z.; SINGHAL, K. Jigsaw: Supporting investigative analysis through interactive visualization. 2007 IEEE Symposium on Visual Analytics Science and Technology, p. 131-138, Oct 2007. Citation on page 56.

TATEMURA, J. Graphical relevance feedback: visual exploration in the document space. Proceedings of 2000 IEEE International Symposium on Visual Languages, p. 39-46, 2000. ISSN 1049-2615. Citations on pages 58 and 60.

TORGERSON, W. S. Multidimensional scaling: I. theory and method. Psychometrika, v. 17, n. 4, p. 401-419, Dec 1952. ISSN 1860-0980. Available: <https://doi.org/10.1007/BF02288916>. Citation on page 38 .

TURNEY, P.; PANTEL, P. From frequency to meaning: Vector space models of semantics. Journal of Artificial Intelligence Research, v. 37, 03 2010. Citations on pages 31 and 32.

WISE, J. A.; THOMAS, J. J.; PENNOCK, K.; LANTRIP, D.; POTTIER, M.; SCHUR, A.; CROW, V. Visualizing the non-visual: spatial analysis and interaction with information from text documents. Proceedings of Visualization 1995 Conference, p. 51-58, 1995. Citation on page 53. 
WONG, P.; THOMAS, J. Visual Analytics. IEEE Computer Graphics and Applications, v. 24, n. 5, p. 20-21, 2004. Citation on page 23.

ZAMANI, H.; CROFT, W. B. Relevance-based word embedding. In: Proceedings of the 40th International ACM SIGIR Conference on Research and Development in Information Retrieval. ACM, 2017. (SIGIR '17), p. 505-514. ISBN 978-1-4503-5022-8. Available: $<$ http://doi.acm.org/10.1145/3077136.3080831>. Citation on page 36.

ZIMMER, B.; SAHLGREN, M.; KERREN, A. Visual analysis of relationships between heterogeneous networks and texts: An application on the ieee vis publication dataset. Informatics, v. 4, p. 11, 05 2017. Citations on pages 49 and 64. 


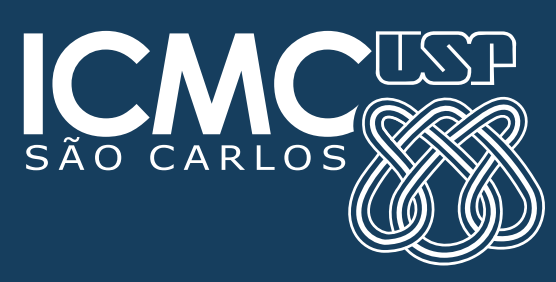

